\title{
PERIODICITY AND CYCLIC HOMOLOGY. PARA-S-MODULES AND PERTURBATION LEMMAS
}

\author{
RAPHAËL PONGE
}

\begin{abstract}
In this paper, we introduce a paracyclic version of $S$-modules. These new objects are called para-S-modules. Paracyclic modules and parachain complexes give rise to para- $S$-modules much in the same way as cyclic modules and mixed complexes give rise to $S$-modules. More generally, para- $S$-modules provide us with a natural framework to get analogues for paracyclic modules and parachain complexes of various constructions and equivalence results for cyclic modules or mixed complexes. The datum of a para- $S$-module does not provide us with a chain complex, and so notions of homology and quasi-isomorphisms do not make sense. We establish some generalizations for para- $S$-modules and parachain complexes of the basic perturbation lemma of differential homological algebra. These generalizations provide us with general recipes for converting deformation retracts of Hoschschild chain complexes into deformation retracts of para- $S$-modules. By using ideas of Kassel this then allows us to get comparison results between the various para- $S$-modules associated with para-precyclic modules, and between them and Connes' cyclic chain complex. These comparison results lead us to alternative descriptions of Connes' periodicity operator. This has some applications in periodic cyclic homology. We also describe the counterparts of these results in cyclic cohomology. In particular, we obtain an explicit way to convert a periodic $(b, B)$-cocycle into a cohomologous periodic cyclic cocycle.
\end{abstract}

\section{INTRODUCTION}

In the terminology of Jones-Kassel [32, 34] an $S$-module is the datum of a chain complex $\left(C_{\bullet}, d\right)$ of modules over some ring $k$ together with a degree -2 chain map $S: C_{\bullet} \rightarrow C_{\bullet-2}$. This notion has its roots in the seminal note of Connes 8 . It naturally comes out in bivariant cyclic theory [8, 15, 32, 34, 47. Incidentally, it encapsulates various approaches to cyclic homology. In particular, we obtain $S$-modules from the cyclic complex and $(b, B)$-bicomplex of Connes [7, 8, 9, from any mixed mixed complex [6, 33, and from the $C C$-bicomplex of Connes [8] and Tsygan [54] with the operators $\left(b,-b^{\prime}, 1-\tau, N\right)$.

A para- $S$-module is similar to an $S$-module where the chain map property $[d, S]=0$ is preserved, but the condition $d^{2}=0$ is relaxed into

$$
d^{2}=(1-T) S,
$$

where $T: C \bullet \rightarrow C$ • is a $k$-linear isomorphism which is compatible with both $d$ and $S$ (see Section 2 for the precise definition). When $T=1$ we recover the definition of an $S$-module. Para- $S$-modules can be also thought of as the unperiodic version of the para-complexes of [55. Indeed, periodizing a para- $S$-module with respect to the $S$-operator precisely gives rise to a para-complex (see Section 21).

The aim of this paper is to lay down the main ground of a paracyclic version of $S$-modules $[8,32$, 34. We call these objects para- $S$-modules. In particular, para- $S$-modules are naturally associated with paracyclic modules and parachain complexes much like $S$-modules are associated with cyclic modules and mixed complexes.

In order to obtain a general apparatus for establishing equivalence results for para- $S$-modules, we generalize to this setting the basic perturbation lemma of differential homological algebra 3 , [17, 22, 53. As we shall see in this paper, this allows us to extend to the paracyclic category various of well known constructions and equivalence results in cyclic homology. In particular, at the level

2010 Mathematics Subject Classification. 19D55.

Key words and phrases. Cyclic homology, perturbation lemmas.

Research supported by Basic Research Grant 2016R1D1A1B01015971 of National Research Foundation of Korea (South Korea). 
of cyclic cohomology this will lead us to an explicit transformation to convert $(b, B)$-cocycles into cohomologous periodic cocycles.

In the follow-up paper [51] the generalized perturbation theory of this paper is used extensively to obtain a constructive version of the Eilenberg-Zilber theorem for bi-paracyclic modules (see also [36). This allows us to get explicit cup and cap products for parachain complexes associated with paracyclic modules. In particular, these constructions are expected to have applications in Hopf cyclic cohomology.

The main results of this paper and of the follow-up paper [51] are important ingredients in the construction of explicit quasi-isomorphisms that compute the cyclic homology of crossed-product algebras associated with actions of (discrete) groups [48, 49, 50].

Examples of para- $S$-modules. A first set of examples of para- $S$-modules consists of para- $S$ modules associated with the parachain complexes of Getzler-Jones [21] (see Section 3). The cyclic complex $\left(C_{\bullet}^{\natural}, b+B u^{-1}\right)$ of a mixed complex $(C \bullet, b, B)$ (see [7, 8, 9, 6, 33) carries a natural $S$ module structure given by the projection $u^{-1}: C_{\bullet}^{\natural} \rightarrow C_{\bullet-2}^{\natural}$. The para-S-module of a parachain complex is defined similarly. Thus, although for a parachain complex we don't get a chain complex in general, we still get a para- $S$-module.

Getzler-Jones 21] also observed that any paracyclic module gives rise to a parachain complex in essentially the same way as a cyclic module gives rise to a mixed complex. This construction actually makes sense for any $H$-unital para-precyclic module (see Proposition 5.3). In our terminology, a para-precyclic module is just a paracyclic module without degeneracies (see Section 6 for the precise definition). Following [34, 57] a precyclic module is called $H$-unital when its bar complex is contractible. We use a similar notion for para-precyclic modules (see Section 5 for the precise definition). In any case, with any $H$-unital para-precyclic module $C$ is associated a para- $S$-module $C^{\natural}$ as the para- $S$-module of its parachain complex. In the cyclic case we recover the total complex of Connes' $(b, B)$-bicomplex of a cyclic module 8 , 9 .

The cyclic homology of a cyclic module $C$ can be also defined by using the total chain complex $\left(C C^{\natural}, \partial+\delta\right)$ of the $C C$-bicomplex This chain complex is turned into an $S$-module by using the shift $u^{-2}: C_{\bullet}^{\text {घ }} \rightarrow C_{\bullet-2}^{\text {घ }}$. This construction actually makes sense in full generality for precyclic modules. In particular, this allows us to define the cyclic homology of non-unital algebras. Given any para-precyclic module $C$, the $C^{\text {घh }}$-construction does not produce a chain complex in general anymore. However, we observe that it still gives rise to a para- $S$-module $C^{\text {虬 }}$ (see Proposition 6.2). Examples of para-precyclic $k$-modules include twisted precyclic modules of non-unital $k$-algebras (see Section 4). Thus, this construction allows us to associate para- $S$-modules with these twisted precyclic modules.

$S$-homotopy equivalences. Because of the relaxation of the condition $d^{2}=0$ into (1.1), the notions of homology and quasi-isomorphisms do not make sense for general para-S-module. However, notions of chain maps, chain homotopies, and chain homotopy equivalences do make sense even when $d^{2} \neq 0$. In the setting of para-S-modules it is natural to require some compatibility with the $(S, T)$-operators. This leads us to define $S$-maps and $S$-homotopies as chain maps and chain homotopies that are compatible with the $(S, T)$-operators. Using these notions we define $S$-homotopy equivalences and $S$-deformation retracts of para-S-modules (see Section 2). Furthermore, any $S$-homotopy equivalence between para- $S$-modules gives rise to a homotopy equivalence between the corresponding periodic para-complexes (see Proposition 2.9).

Chain homotopy equivalences are stronger notions of equivalence and enjoy better functorial properties than quasi-isomorphisms. Therefore, even between $S$-modules, it is quite significant to have chain homotopy equivalences.

Quasi- $S$-modules. It is natural to look at para- $S$-modules that are equivalent to $S$-modules. We single out a sub-class of para- $S$-modules, called quasi-S-modules, which admit $S$-deformation retracts to $S$-modules (see Section 2 for their precise definition). This leads us to define quasimixed complexes and quasi-precyclic modules as the parachain complexes and para-precyclic modules whose para- $S$-modules are quasi- $S$-modules. In fact, for these notions the sole splitting 
$C_{\bullet}=\operatorname{ker}(1-T) \oplus\left(C_{\bullet} / \operatorname{ran}(1-T)\right)$ ensures us to get quasi-S-modules (see Proposition 3.7 and Proposition 6.6). In particular, we recover the quasi-cyclic modules of [37, 38, (cf. Section 4).

Examples of quasi-(pre)cyclic modules include the $r$-(pre)cyclic modules of Feigin-Tsygan [19, Appendix], the twisted (pre)cyclic modules associated with periodic automorphisms and the twisted group cyclic modules associated with finite order central elements (see Section 4). More generally, we obtain a quasi-mixed complex or a quasi-(pre)cyclic module as soon the $T$-operator solves a polynomial equation $Q(T)=0$ with $Q(1)=1$ (see Lemma 3.3 and Lemma 4.14).

Given any quasi-mixed complex $C=(C, b, B)$, we observe that a simple modification of the $B$-differential allows us to get a mixed complex $C=\left(C_{\bullet}, b, \tilde{B}\right)$ whose $S$-module is $S$-homotopy equivalent to the para- $S$-module $C^{\natural}$ (see Proposition 3.10). Similarly, when $C$ is a quasi-precyclic module, we can modify the construction of the para- $S$-module $C^{\text {घh }}$ to get an $S$-module $\widetilde{C}^{\text {घh }}$ which is

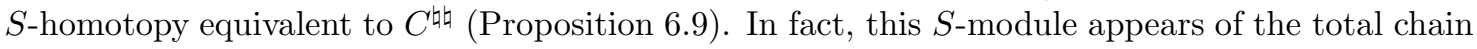
complex of a chain bicomplex, which is constructed like the $C C$-bicomplex only by modifying the $N$-operator. In particular, for $r$-(pre)cyclic modules we recover the bicomplex of Feigin-Tsygan [19, Appendix].

Perturbation lemmas. An important tool in cyclic homology is Connes' long exact sequence, which relates cyclic homology to Hochschild homology [7, 8, 9]. In particular, a map of mixed complexes (and more generally an $S$-map) is a quasi-isomorphism at the cyclic and periodic levels as soon as it is a quasi-isomorphism at the Hochschild level. As observed by Kassel [34, in the setting of mixed complexes the basic perturbation lemma [3, 17, 22, 53] often allows us to convert a deformation retract of Hochschild complexes into an $S$-deformation retract of cyclic complexes. In particular, a number of well known quasi-isomorphisms in cyclic homology can be reformulated as $S$-deformation retracts of $S$-modules (see [1, 34]).

We seek for extending Kassel's approach to parachain complexes and para-(pre)cyclic modules. To this end we generalize the basic perturbation lemma to "para-twin" complexes (Lemma 7.1). By a para-twin complex we just mean a graded $k$-module together with a pair of $k$-linear maps of degree -1 , which are not assumed to be differentials (see Section 7 for the precise definition).

In this generalized setting the input data include a mere pair of left/right chain homotopy inverses which need not be chain maps or anti-sided inverses, whereas for the basic perturbation lemma the input data actually involve a true deformation retract provided by a pair of chain maps. However, as for the basic perturbation lemma, the chain homotopy equivalences are required to be "special" (see Section for the precise meaning). Unlike with the basic perturbation lemma this assumption is essential ( $c f$. Remark (7.6). Nevertheless, under suitable conditions this requirement can be relaxed (see Lemma 7.7).

Specializing these generalized perturbation lemmas to parachain complexes provides us with general recipes for converting deformation retracts of Hoschschild chain complexes into $S$-deformation retracts of para- $S$-modules (see Lemma 7.9, Lemma 7.13, Lemma 7.14, and Lemma 7.15). In particular, this provides us with a substitute for Connes' long exact sequence in the framework of parachain complexes.

Comparing $C^{\natural \natural}$ and $C^{\natural}$. For any $H$-unital precyclic $k$-module $C$, we have three cyclic chain complexes: the cyclic complex $C^{\lambda}$, the $C^{\natural}$-complex of its mixed complex, and the total complex $C^{\natural \natural}$ of the $C C$-bicomplex. The last two chain complexes are quasi-isomorphic, and, when $k \supset \mathbb{Q}$, they are both quasi-isomorphic to the $C^{\lambda}$-complex (see [8, 9, 43, 54]). By using suitable versions of the basic perturbation lemma, Kassel 34 further exhibited a deformation retract of $C^{\text {约 }}$ to $C^{\natural}$ and, when $k \supset \mathbb{Q}$, he also constructed a deformation retract of $C^{\text {如 }}$ to $C^{\lambda}$.

We seek for extending Kassel's results to the paracyclic setting by using the generalized perturbation theory of this paper. We start by showing that, when $C$ is an $H$-unital para-precyclic $k$-module, the para- $S$-module $C^{\natural}$ is an $S$-deformation retract of $C^{\natural \natural}$ (Proposition 8.3). In particular, as pointed out in [34, the $B$-operator naturally re-appears from this process. In the precyclic case, we recover the deformation retract of [34]. 
Comparing $C^{\text {蚂 }}$ and $C^{\lambda}$. We also compare the para- $S$-module $C^{\text {घh }}$ to the cyclic complex $C^{\lambda}$ when $k \supset \mathbb{Q}$. The latter actually makes sense for any para-precyclic module. Given any paraprecyclic $k$-module $C$ (with $k \supset \mathbb{Q}$ ), we show that $C^{\lambda}$ is a deformation retract of $C_{T}^{\text {叫 }}$, where $C_{T}$ is the pre-cyclic $k$-module obtained by mod-outing $C$ by $\operatorname{ran}(1-T)$ (see Proposition 9.5).

A difference with the approach of [34] lies on the use of the generalized perturbation theory of this paper. This allows us to construct a $k$-linear map $\nu_{0}^{\text {蛞 }}: C_{\bullet} \rightarrow C_{\bullet}^{\text {घ曰 }}$, which is "almost" a chain homotopy inverse of the canonical projection $\pi_{0}^{\text {घh }}: C_{\bullet}^{\text {घh }} \rightarrow C_{\bullet}$. Namely, this is a chain homotopy left-inverse, and a right-inverse modulo $\operatorname{ran}(1-\tau)$, as well as a chain map modulo $\operatorname{ran}(1-T)$ (see Lemma 9.3). It then descends to a chain map $\bar{\nu}^{\text {吅 }}: C_{\bullet}^{\lambda} \rightarrow C_{T, \bullet}^{\text {叫 }}$, which is a chain homotopy

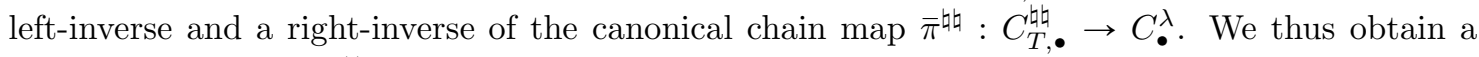
deformation retract $C_{T}^{\text {घh }}$ to $C^{\lambda}$. Incidentally, our approach avoid using the cyclic relation $T=1$, which is used in [34, but is not available in general in the para-precyclic setting.

When $C$ is quasi-precyclic the map $\nu_{0}^{\text {घh }}$ also gives rise to a right-inverse and chain homotopy

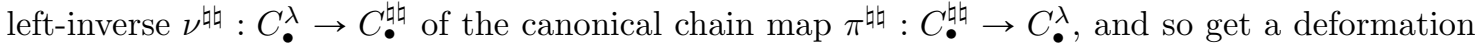
retract of $C^{\text {蚂 }}$ to $C^{\lambda}$ (see Proposition 9.5).

Comparing $C^{\natural}$ and $C^{\lambda}$. When $C$ is a $H$-unital pre-paracyclic $k$-module with $k \supset \mathbb{Q}$, we can combine the previous comparison results to compare the para- $S$-module $C^{\natural}$ to the cyclic complex $C^{\lambda}$. More precisely, we obtain a $k$-linear map $\nu_{0}^{\natural}: C_{\bullet} \rightarrow C_{\bullet}^{\natural}$ which, in the same way as the map $\nu_{0}^{\natural \natural}$ above, is an almost chain homotopy inverse of the canonical projection $\pi_{0}^{\natural}: C_{\bullet}^{\natural} \rightarrow C$. (see Lemma 10.1). It then descends to a chain map $\bar{\nu}^{\natural}: C^{\lambda} \rightarrow C_{T, \bullet}^{\natural}$, which a right-inverse and chain homotopy left-inverse of the canonical map $\bar{\pi}^{\natural}: C_{T, \bullet}^{\natural} \rightarrow C^{\lambda}$. This provides us with a deformation retract of $C_{T}^{\natural}$ to $C^{\lambda}$ (Proposition 10.2).

When $C$ is quasi-cyclic the map $\nu_{0}^{\natural}$ also gives rise to a right-inverse and chain homotopy leftinverse $\nu^{\natural}: C^{\lambda} \rightarrow C_{\bullet}^{\natural}$ of the canonical chain map $\pi^{\natural}: C_{\bullet}^{\natural} \rightarrow C_{\bullet}^{\lambda}$, and so this gives a deformation retract of $C^{\natural}$ to $C^{\lambda}$ (Proposition 10.4).

Periodicity operator. When $C$ is a precyclic $k$-module with $k \supset \mathbb{Q}$, Kassel 34 used the deformation retract of $C^{\text {घh }}$ to $C^{\lambda}$ to get an alternative description of periodicity operator of Connes [7, 9 , in cyclic homology. We seek for extending Kassel's approach to the pre-paracyclic setting. One feature of Kassel's approach is the use of a nilpotent chain homotopy. Such a chain homotopy need not be available in the paracyclic setting (see Remark 7.6 on this point). This issue is bypassed by using the almost chain homotopy inverse $\nu_{0}^{\text {印 }}$ described above. Namely, let $S_{0}: C_{\bullet} \rightarrow C_{\bullet-2}$ be the $k$-linear map defined by

$$
S_{0}(x)=\pi_{0}^{\text {蚛 }}\left(u^{-2} \nu_{0}^{\text {蚛 }}(x)\right), \quad x \in C_{\bullet} .
$$

Then $S_{0}$ descends to a chain map $S: C_{\bullet}^{\lambda} \rightarrow C_{\bullet_{-2}}^{\lambda}$ that turns the cyclic complex $C^{\lambda}$ into an $S$-module and with respect to which the chain homotopy inverse $\bar{\nu}^{\text {如 }}: C_{\bullet}^{\lambda} \rightarrow C_{T, \bullet}^{\text {姉 }}$ is an $S$-map and

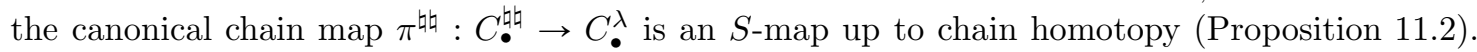
There is a unique such map (loc. cit.). When $C$ is quasi-precyclic it can be also shown that the

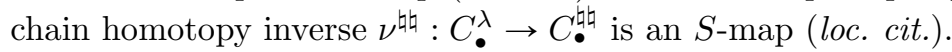

We also compute the $S$-operator and shows it actually agrees with Connes' periodicity operator in the precyclic case (Proposition 11.8). This equality occurs at the level of chains, rather than at the homology level (compare [42, 34]). In addition, we obtain a new, and somewhat concise, formula for the $S$-operator (loc. cit.).

When $C$ is $H$-unital, it can be shown that the $S$-operator is induced on $C_{\bullet}^{\lambda}$ by the operator $x \rightarrow \pi_{0}^{\natural}\left(u^{-1} \nu_{0}^{\natural}(x)\right)$ (Proposition 11.5). The chain homotopy inverse $\bar{\nu}^{\natural}: C_{\bullet}^{\lambda} \rightarrow C_{T, \bullet}^{\natural}$ is an $S$-map and the canonical chain map $\pi^{\natural}: C_{\bullet}^{\natural} \rightarrow C_{\bullet}^{\lambda}$ is an $S$-map up to chain homotopy (loc. cit.). When $C$ is quasi-precyclic (and $H$-unital) the chain homotopy inverse $\nu^{\natural}: C_{\bullet}^{\lambda} \rightarrow C_{\bullet}^{\natural}$ is an $S$-map as well (loc. cit.).

The description of the periodicity operator for $H$-unital precyclic modules above has some interesting implications in periodic cyclic homology. First, Connes [9] proved a formula that 
relates explicitly the periodicity operator to the $(b, B)$-differentials in cyclic cohomology. By using the description the $S$-operator in terms of the map $\nu_{0}^{\natural}$ allows us to a new proof of a dual version of Connes' formula (see Proposition [11.9).

Second, any $S$-map between $S$-modules that is a quasi-isomorphism gives rise to a quasiisomorphism in periodic cyclic homology (see Section 2). Thus, the very fact that the quasiisomorphism $\bar{\nu}^{\natural}: C_{\bullet}^{\lambda} \rightarrow C_{T}^{\natural}$ • is an $S$-map ensures us it extends to a quasi-isomorphism $\bar{\nu}^{\sharp}$ : $C_{\bullet}^{\lambda, \sharp} \rightarrow C_{T, \bullet}^{\sharp}$, where $C_{T}^{\sharp}$ is the periodic complex of the $H$-unital precyclic module $C_{T}$ and $C_{\bullet}^{\lambda, \sharp}$ is the inverse limit of the modules $C_{\bullet}^{\lambda}$ under the periodicity operator (see Proposition [11.10).

Applications in cyclic cohomology. By duality, the above results have interesting counterparts in cyclic cohomology and periodic cyclic cohomology. First, we obtain an explicit way to convert a $(b, B)$-cocycle into a cohomologous cyclic cocycle (see Proposition 12.1 and Corollary 12.3).

Second, we exhibit an explicit quasi-isomorphism that realizes Connes' isomorphism between periodic cyclic cohomology and the direct limit of cyclic cohomology under the periodicity operator (Proposition 12.4). More precisely, this quasi-isomorphism allows us to invert the natural map in cohomology provided by the inclusion of cyclic cochain complex into the periodic cyclic cochain complex. This might have some applications to the representation of the Connes-Chern character by $(b . B)$-cocycles ( $c f$. Remark 12.5).

Organization of the paper. The rest of the paper is organized as follows. In Section 2, we present the main definitions regarding para- $S$-modules, quasi- $S$-modules, and $S$-homotopy equivalences, as well as some of their properties. In Section [3, we present the construction of the $S$-module of a parachain complex by following [21] and show that we obtain a quasi- $S$-module in the case of a quasi-mixed complex. In Section 4 after recalling basic facts on paracyclic modules, we introduce para-precyclic modules and quasi-precyclic modules. In Section 5 , by elaborating on the considerations of [21, 34] we explain how to associate a parachain complex with any $\mathrm{H}$ unital para-precyclic module. In Section 6, we construct the para- $S$-module $C^{\text {th }}$ of an arbitrary para-precyclic module $C$ and show that we obtain a quasi- $S$-module when $C$ is quasi-precyclic. In Section 7 we establish some generalizations of the basic perturbation lemma to para-twin complexes and parachain complexes. In Section 8 , we show that when $C$ is an $H$-unital para-precyclic module, the para-S-module $C^{\natural}$ is an $S$-deformation retract of $C^{\text {घh }}$. In Section 9 we show that, when $C$ is a para-precyclic module and the ground ring contains $\mathbb{Q}$, we compare the para- $S$-module $C^{\text {蚂 }}$ to the cyclic complex $C^{\lambda}$. In Section 10, we show that, when $C$ is an $H$-unital para-precyclic module and the ground ring contains $\mathbb{Q}$, we also can compare the para-S-module $C^{\natural}$ to $C^{\lambda}$. In Section 11, we use these comparison results to describe Connes' periodicity operator and gives some applications in periodic cyclic homology. Finally, in Section 12 we describe the counterparts of these results in cyclic cohomology and periodic cyclic cohomology.

Notation. Throughout this paper we denote by $k$ a ring with unit which is not assumed to be commutative. Unless otherwise mentioned in the last section, by a $k$-module we shall mean a left $k$-module.

Acknowledgements. The author would like to thank Alain Connes, Ulrich Krähmer, Henri Moscovici, Bahram Rangipour, and Christian Voigt for discussions related to the subject matter of this paper. He also thanks McGill University, University of New South Wales and University of Québec at Montréal for their hospitality during the preparation of this paper.

\section{PARA-S-Modules}

In this section, we introduce para- $S$-modules as the natural "para" version of the $S$-modules of Jones-Kassel [32, 34]. We shall also look at chain homotopy equivalences and deformation retracts between para- $S$-modules. This will provide us with a natural notion of equivalence between para$S$-modules. 
2.1. $S$-modules. In the terminology of Jones-Kassel 32,34 an $S$-module is given by chain complex of $k$-modules $\left(C_{\bullet}, d\right)$ and a chain map $S: C \bullet \rightarrow C_{\bullet-2}$. This notion has its roots in the seminal note of Connes [8] and it naturally comes into play in bivariant cyclic homology [8, 15, 32, 34, 47]. Incidentally, it encapsulates various approaches to cyclic homology. In particular, examples of $S$-modules include the following:

- The cyclic complex of a (pre-)cyclic module of Connes 7, 8, 9, (see also Section 9).

- The total complex of the $(b, B)$-bicomplex of Connes [7, 8, 9, and more generally the cyclic complex of a mixed complex in the sense of Burghelea [6] and Kassel [33] (see also Section 3 .

- The total complex of the $C C$-bicomplex with the operators $\left(b,-b^{\prime}, 1-\tau, N\right)$ of Connes 8 , Section 4] and Tsygan [54, Proposition 1] (see also Section [6).

We will describe generalizations for these examples in subsequent sections. Following is a further example of an $S$-module.

Example 2.1. Given a group $\Gamma$, let us denote by $k \Gamma$ its group ring over $k$. We let $C(\Gamma)=(C \bullet(\Gamma), \partial)$ be its standard complex of $k \Gamma$-modules, where $C_{m}(\Gamma)=k \Gamma^{m+1}$ and the boundary $\partial: C_{\bullet}(\Gamma) \rightarrow$ $C_{\bullet+1}(\Gamma)$ is given by

$$
\partial\left(\gamma_{0}, \ldots, \gamma_{m}\right)=\sum_{0 \leqslant j \leqslant m}(-1)^{j}\left(\gamma_{0}, \ldots, \widehat{\gamma_{j}}, \ldots, \gamma_{m}\right), \quad \gamma_{j} \in \Gamma,
$$

where $\hat{\cdot}$ denotes omission. Let $u$ be a group 2-cocycle, i.e., a $\Gamma$-equivariant map $u: C_{2}(\Gamma) \rightarrow k$ such that $u \circ \partial=0$. The cap product $u \frown-: C \bullet(\Gamma) \rightarrow C_{\bullet}-2(\Gamma)$ is given by

$$
u \frown\left(\gamma_{0}, \ldots, \gamma_{m}\right)=u\left(\gamma_{0}, \gamma_{1}, \gamma_{2}\right)\left(\gamma_{2}, \ldots, \gamma_{m}\right), \quad \gamma_{j} \in \Gamma .
$$

This is a chain map, and so $(C \bullet(\Gamma), \partial, u \frown-)$ is an $S$-module. This kind of $S$-module naturally appears in the description of the cyclic homology of the group ring $k \Gamma$ (see [5, 31, 45]).

Any $S$-module $C=\left(C_{\bullet}, d, S\right)$ gives rise to a chain complex $\left(C_{\bullet}^{\sharp}, d\right)$, where $C_{m}^{\sharp}=\lim _{S} C_{m+2 q}$, $m \geqslant 0$, and $\lim _{S}$ is the limit of the inverse system defined by the operators $S: C_{m+2 q+2} \rightarrow C_{m+2 q}$, $q \geqslant 0$. Namely,

$$
C_{m}^{\sharp}=\left\{\left(x_{m+2 q}\right)_{q \geqslant 0} ; S x_{m+2 q+2}=x_{m+2 q} \forall q \geqslant 0\right\} .
$$

Moreover, as $S$ is a chain map, the differential $d$ is compatible with the $S$-operator, and so it gives rise to a $k$-linear differential $d: C_{\bullet}^{\sharp} \rightarrow C_{\bullet-1}^{\sharp}$.

We have an identification $C_{m}^{\sharp} \simeq C_{m+2}^{\sharp}$ given by the shift $\left(x_{m}, x_{m+2}, \ldots\right) \rightarrow\left(x_{m+2}, x_{m+4}, \ldots\right)$ and its inverse $\left(x_{m+2}, \ldots\right) \rightarrow\left(S x_{m+2}, x_{m+2}, \ldots\right)$. Thus, if we set $m=2 p+i$ with $i \in\{0,1\}$, then we have a natural identification $C_{m}^{\sharp} \simeq C_{i}^{\sharp}$. Under this identification the chain complex $\left(C_{\bullet}^{\sharp}, d\right)$ is 2-periodic and is naturally identified with the $\mathbb{Z}_{2}$-graded chain complex $C^{\sharp}:=\left(C_{0}^{\sharp} \oplus C_{1}^{\sharp}, d\right)$, where $d$ is regarded as an odd $k$-linear operator on $C_{0}^{\sharp} \oplus C_{1}^{\sharp}$, i.e., it maps $C_{0}^{\sharp}$ (resp., $C_{1}^{\sharp}$ ) to $C_{1}^{\sharp}$ (resp., $C_{0}^{\sharp}$ ). (This uses the identification $C_{2}^{\sharp} \simeq C_{0}^{\sharp}$ described above.)

We call the $\mathbb{Z}_{2}$-graded complex $C^{\sharp}$ the periodic chain complex of the $S$-module $C$. If we denote by $H_{\bullet}(C)$ (resp., $\left.H_{\bullet}\left(C^{\sharp}\right)\right)$ the homology of $C$ (resp., $\left.C^{\sharp}\right)$, then the operator $S$ induces a $k$-linear map $S: H_{\bullet}(C) \rightarrow H_{\bullet-2}(C)$, and we have an exact sequence,

$$
0 \longrightarrow \lim _{S}^{1} H_{\bullet}(C) \longrightarrow H_{\bullet}\left(C^{\sharp}\right) \longrightarrow \lim _{S} H_{\bullet}(C) \longrightarrow 0,
$$

where $\lim ^{1}$ is the first derived functor of the inverse limit functor $\lim _{(\text {see, e.g., }[42,56}^{56}$ ).

In the terminology of Kassel [33, 34, given $S$-modules $C=\left(C_{\bullet}, d, S\right)$ and $\bar{C}=\left(\bar{C}_{\bullet}, d, S\right)$, an $S$-map is any chain map $f: C_{\bullet} \rightarrow \bar{C}_{\bullet}$ that is compatible with the $S$-operators. Any $S$-map $f: C_{\bullet} \rightarrow \bar{C}_{\bullet}$ gives rise to a chain map $f^{\sharp}: C_{\bullet}^{\sharp} \rightarrow \bar{C}_{\bullet}^{\sharp}$ between the corresponding periodic complexes. More generally, any $S$-compatible $k$-linear map $f: C_{\bullet} \rightarrow \bar{C}_{\bullet+d}$ of degree $d, d \in \mathbb{Z}$, gives rise to $k$-linear map between the periodic complexes which is even or odd according to the parity of $d$. By using the functoriality of the exact sequence (2.3) and the 5-lemma we obtain the following result. 
Proposition 2.2. Let $f: C_{\bullet} \rightarrow \bar{C}_{\bullet}$ be an $S$-map and a quasi-isomorphism. Then the chain map $f^{\sharp}: C_{\bullet}^{\sharp} \rightarrow \bar{C}_{\bullet}^{\sharp}$ is a quasi-isomorphism as well.

2.2. Para $S$-modules. The paracyclic category of Feigin-Tsygan [19] and Getzler-Jones 21] (see also [16, 18]) appears in various computations of cyclic homology groups, including the cyclic homology of group rings and crossed-product algebras associated with group actions on $k$-algebras (see, e.g., [5, 13, 20, 21, 44, 48]). Getzler-Jones also introduced parachain complexes as the "para" version of mixed complexes (see also [16]). In this paper, we shall use the following "para" generalization of $S$-modules.

Definition 2.3. A para-S-module is given by a system $\left(C_{\bullet}, d, S, T\right)$, where $C_{m}, m \geqslant 0$, are $k$ modules, $d: C_{\bullet} \rightarrow C_{\bullet-1}$ and $S: C_{\bullet} \rightarrow C_{\bullet-2}$ are $k$-linear maps, and $T: C_{\bullet} \rightarrow C_{\bullet}$ is a $k$-linear isomorphism in such a way that

$$
d^{2}=S(1-T) \quad \text { and } \quad[d, S]=[d, T]=[S, T]=0 .
$$

Remark 2.4. When $T=1$ we recover the definition of an $S$-module. In particular, any $S$-module is a para- $S$-module.

Remark 2.5. As we shall see later, para-S-modules provides us with a natural setting for the following constructions:

- A version for parachain complexes of the cyclic complex of a mixed complex. This construction goes back to Getzler-Jones [21] (see Section 3).

- A version for para-precyclic $k$-modules of (the total complex of) the $C C$-bicomplex (see Section 6).

As we shall see, although these constructions do not provide us with chain complexes, they do give rise to para- $S$-modules.

Given para-S-modules $C=\left(C_{\bullet}, d, S, T\right)$ and $\bar{C}=\left(\bar{C}_{\bullet}, d, S, T\right)$, we shall say that a $k$-linear map $f: C_{\bullet} \rightarrow \bar{C}_{\bullet}$ is a chain map when it is compatible with the $d$-operators. We shall say that this is a para- $S$-module map, or simply an $S$-map, when it is further compatible with the operators $(S, T)$. When $T=1$ we recover the usual notion of an $S$-map between $S$-modules ([32, 33]).

As $\left(C_{\bullet}, d\right)$ and $\left(\bar{C}_{\bullet}, d\right)$ need not be chain complexes, we cannot speak about quasi-isomorphisms between them. However, notions of chain homotopy equivalences and deformation retracts do make sense in the same way as with usual chain complexes. More precisely, we shall say that two chain maps $f_{2}: C_{\bullet} \rightarrow \bar{C}_{\bullet}$ and $f_{2}: C_{\bullet} \rightarrow \bar{C}_{\bullet}$ are chain homotopic when there is a $k$-linear map $\varphi: C_{\bullet} \rightarrow \bar{C}_{\bullet+1}$ such that

$$
f_{1}-f_{2}=d \varphi+\varphi d
$$

Given chain maps $f: C_{\bullet} \rightarrow \bar{C}_{\bullet}$ and $g: \bar{C}_{\bullet} \rightarrow C_{\bullet}$, we then say that $g$ is a homotopy inverse of $f$ when $g f$ and $f g$ are chain homotopy equivalent to the identity maps of $C_{\bullet}$ and $\bar{C}_{\bullet}$. We similarly define notions of chain homotopy left-inverse and right-inverse.

Definition 2.6. A chain homotopy equivalence between $C$ and $\bar{C}$ is given by chain maps $f$ : $C_{\bullet} \rightarrow \bar{C}_{\bullet}$ and $g: \bar{C}_{\bullet} \rightarrow C_{\bullet}$ that are chain homotopy inverses of each other. We have a deformation retract of $C$ to $\bar{C}$ when we can choose $f$ and $g$ so that $f g=1$.

In what follows we will be interested in chain homotopies that are compatible with the $(S, T)$ operators. We shall say that $S$-maps $f: C_{\bullet} \rightarrow \bar{C}_{\bullet}$ and $f^{\prime}: C_{\bullet} \rightarrow \bar{C}_{\bullet}$ are $S$-homotopic when they are chain homotopic and the homotopy (2.5) can be realized by means of a chain homotopy $\varphi: C_{\bullet} \rightarrow \bar{C}_{\bullet+1}$ that is compatible with the $(S, T)$-operators. This allows us to give sense to notions of $S$-homotopy inverses of $S$-maps.

Definition 2.7. An $S$-homotopy equivalence between $C$ and $\bar{C}$ is given by chain maps $f: C \bullet \rightarrow \bar{C}$ • and $g: \bar{C}_{\bullet} \rightarrow C$. that are $S$-homotopy inverses of each other. We have an $S$-deformation retract of $C$ to $\bar{C}$ when we can choose $f$ and $g$ so that $f g=1$. 
2.3. The Periodic para-complex of a para- $S$-module. Let $C=\left(C_{\bullet}, d, S, T\right)$ be a para- $S$ module. In the same way as with $S$-modules, the operator $S$ allows us to form a system $\left(C_{0}^{\sharp} \oplus C_{1}^{\sharp}, d\right)$, where the $\mathbb{Z}_{2}$-graded $k$-module $C_{0}^{\sharp} \oplus C_{1}^{\sharp}$ is defined as in (2.2) and the $k$-linear map $d: C_{\bullet}^{\sharp} \rightarrow C_{\bullet-1}^{\sharp}$ is induced from the "para-differential" $d: C \bullet \rightarrow C_{\bullet-1}$. The compatibility of $T$ with $S$ also implies that it gives rise to an invertible (even) $k$-linear map $T: C_{\bullet}^{\sharp} \rightarrow C_{\bullet}^{\sharp}$. Moreover, given any sequence $\left(x_{0}, x_{2}, \ldots\right) \in C_{0}^{\sharp}$, we have

$$
d^{2}\left(x_{0}, x_{2}, \ldots\right)=d\left(d x_{2}, d x_{4}, \ldots\right)=\left(d^{2} x_{2}, d^{2} x_{4}, \ldots\right) .
$$

As $d^{2} x_{2 q+2}=(1-T) S x_{2 q+2}=(1-T) x_{2 q}$, we see that $d^{2}=(1-T)$ on $C_{0}^{\sharp}$. Likewise, $d^{2}=(1-T)$ on $C_{1}^{\sharp}$. As $T: C_{\bullet}^{\sharp} \rightarrow C_{\bullet}^{\sharp}$ is invertible, this shows that the triple $\left(C_{\bullet}^{\sharp}, d, T\right)$ is a para-complex in the sense of Voigt [55].

Definition 2.8. The para-complex $C^{\sharp}:=\left(C_{\bullet}^{\sharp}, d, T\right)$ is called the periodic para-complex of the para- $S$-module $C$.

Given para-S-modules $C=\left(C_{\bullet}, d, S, T\right)$ and $\bar{C}=\left(\bar{C}_{\bullet}, d, S, T\right)$, any $S$-map $f: C_{\bullet} \rightarrow \bar{C}_{\bullet}$ gives rise to a $T$-compatible chain map $f^{\sharp}: C_{\bullet}^{\sharp} \rightarrow \bar{C}_{\bullet}^{\sharp}$. More generally, any $k$-linear map $f: C_{\bullet} \rightarrow \bar{C}_{\bullet+d}$, $d \in \mathbb{Z}$, which is compatible with the operators $S$ and $T$ gives to a $T$-compatible $k$-linear map $f^{\sharp}$ between periodic chains. We get an even or odd map according to the parity of $d$.

In what follows we shall call $T$-homotopy any chain homotopy between para-complexes that is realized by means of $T$-compatible $k$-linear maps. Any $S$-homotopy between $C$ and $\bar{C}$ gives rise a $T$ homotopy between $C_{\text {. }}^{\sharp}$ and $\bar{C}_{\bullet}^{\sharp}$. Therefore, any $S$-homotopy equivalence between paras- $S$-modules gives rise to a $T$-homotopy equivalence between the corresponding periodic para-complexes. More precisely, we have the following result.

Proposition 2.9. Let $f: C_{\bullet} \rightarrow \bar{C}$. and $g: \bar{C} \rightarrow C$. be $S$-maps such that

$$
f g=1+d \psi+\psi d, \quad g f=1+d \varphi+\varphi d,
$$

where $\varphi: C_{\bullet} \rightarrow C_{\bullet+1}$ and $\psi: \bar{C}_{\bullet} \rightarrow \bar{C}_{\bullet+1}$ are T-compatible $k$-linear maps. Then, we have

$$
f^{\sharp} g^{\sharp}=1+d \psi^{\sharp}+\psi^{\sharp} d, \quad g^{\sharp} f^{\sharp}=1+d \varphi^{\sharp}+\varphi^{\sharp} d .
$$

In particular, this gives a T-homopotopy equivalence between $C^{\sharp}$ and $\bar{C}^{\sharp}$. We get a deformation retract when $f g=1$.

Remark 2.10. By Proposition 2.2, when $C$ and $\bar{C}$ are both $S$-modules, any quasi-isomorphism provided by an $S$-map $f: C_{\bullet} \rightarrow \bar{C}_{\bullet}$ gives rise to quasi-isomorphism $f^{\sharp}: C_{\bullet}^{\sharp} \rightarrow \bar{C}_{\bullet}^{\sharp}$. Proposition 2.9 provides us with some substitute for this result in the framework of para- $S$-modules.

2.4. Property (DR) and quasi-S-modules. Given any para- $S$-module $C=(C \bullet, d, S, T)$ we set

$$
C_{\bullet}^{T}=\operatorname{ker}(1-T), \quad R_{\bullet}^{T}=\operatorname{ran}(1-T), \quad C_{T, \bullet}=C_{\bullet} / R_{\bullet}^{T} .
$$

The compatibility of $T$ with the operators $d$ and $S$ implies that these operators induce operators on $C_{\bullet}^{T}$ and $R_{\bullet}^{T}$, so that we obtain para- $S$-modules $C^{T}:=\left(C_{\bullet}^{T}, d, S, T\right)$ and $R^{T}:=\left(R_{\bullet}^{T}, d, S, T\right)$. In fact, as $T=1$ on $C_{\bullet}^{T}$, we see that $C^{T}$ is actually an $S$-module.

The compatibility of $T$ with the operators $d$ and $S$ also implies that these operators descend to operators on $C_{T, \bullet}$. As $T=1$ on $C_{T, \bullet}$ we obtain another $S$-module $C_{T}:=\left(C_{T, \bullet}, d, S\right)$. Note that the canonical projection $\pi_{T}: C_{\bullet} \rightarrow C_{T, \bullet}$ is an $S$-map.

We further observe that, given para- $S$-modules $C=\left(C_{\bullet}, d, S, T\right)$ and $\bar{C}=\left(\bar{C}_{\bullet}, d, S, T\right)$, any $S$-map $f: C_{\bullet} \rightarrow \bar{C}_{\bullet}$ induces an $S$-map $f: C_{\bullet}^{T} \rightarrow \bar{C}_{\bullet}^{T}$ and it descends to a unique $S$-map $f: C_{T, \bullet} \rightarrow \bar{C}_{T, \bullet}$. More generally, any $S$-homotopy equivalence between $C$ and $\bar{C}$ gives rise to $S$-homotopy equivalences between the $S$-modules $C^{T}$ and $\bar{C}^{T}$ and between $C_{T}$ and $\bar{C}_{T}$.

We are especially interested in para- $S$-modules with the following property.

Definition 2.11. A para- $S$-module $C$ has property (DR) when the canonical projection $\pi_{T}$ : $C \bullet \rightarrow C_{T, \bullet}$ gives rise to an $S$-deformation retract (i.e., it has a right-inverse which an $S$-homotopy left-inverse). 
In what follows, given any $x \in C_{\bullet}$, it will be convenient to set $\bar{x}=\pi_{T}(x)$. We shall also say that a para-S-module is $S$-contractible when the identity map is $S$-homotopic to 0 .

We have the following characterization of property (DR).

Proposition 2.12. Let $C=(C \bullet, d, S, T)$ be a para-S-module. Then $C$ has property (DR) if and only the following two conditions are satisfied:

(i) The para-S-module $R^{T}$ is $S$-contractible.

(ii) There is a sub-S-module $C^{\prime} \subset C^{T}$ such that $C_{\bullet}=C_{\bullet}^{\prime} \oplus R_{\bullet}^{T}$.

Proof. Suppose that $C$ has property (DR). This means there are a chain map $\iota: C_{T, \bullet} \rightarrow C_{\bullet}$ and an $(S, T)$-compatible $k$-linear map $h: C \bullet \bullet C_{\bullet+1}$ such that $\pi_{T} \iota=1$ and $\iota \pi_{T}=1+d h+h d$. The $(S, T)$-compatibility of $h$ implies that it induces an $(S, T)$-compatible $k$-linear map $h: R_{\bullet}^{T} \rightarrow R_{\bullet+1}^{T}$. Moreover, the equality $1+d h+d h=\iota \pi_{T}$ implies that $1+d h+d h=0$ on $R_{\bullet}^{T}=\operatorname{ker} \pi_{T}$. Thus, the identity map on $R_{\bullet}^{T}$ is $S$-homotopic to 0, i.e., $R^{T}$ is $S$-contractible.

Set $\pi=\iota \pi_{T}$ and $C_{\bullet}^{\prime}=\operatorname{ran} \pi$. The $T$-compatibility of $\iota$ means that $T \iota=\iota$, and so $T \pi=\pi$. This means that $C_{\bullet}^{\prime}=\operatorname{ran} \pi$ is contained in $C_{\bullet}^{T}$. In addition, the operators $d$ and $S$ acts on $C_{\bullet}^{\prime}$. Thus, we get a sub-S-module $C^{\prime}=\left(C_{\bullet}^{\prime}, d, S, T\right)$ of $C^{T}$. As $\pi_{T} \iota=1$, we have $\pi^{2}=\iota(\pi \iota) \pi_{T}=\pi$, and so $\pi$ is a projection. This implies that $C_{\bullet}=C_{\bullet}^{\prime} \oplus \operatorname{ker} \pi$. As $\pi=\iota \pi_{T}$ it is immediate that $\operatorname{ker} \pi$ contains $\operatorname{ker} \pi_{T}$. Moreover, as $\pi_{T} \pi=\left(\pi_{T} \iota \pi\right) \pi_{T}=\pi_{T}$, we also see that $\operatorname{ker} \pi \subset \operatorname{ker} \pi_{T}$. Thus, $\operatorname{ker} \pi$ agrees with ker $\pi_{T}=R_{\bullet}^{T}$, and hence $C_{\bullet}=C_{\bullet}^{\prime} \oplus R_{\bullet}^{T}$.

Conversely, suppose that the condition (i) and (ii) are satisfied. The latter condition means there is a a sub-S-module $C^{\prime} \subset C^{T}$ such that $C_{\bullet}=C_{\bullet}^{\prime} \oplus R_{\bullet}^{T}$. Let $\pi^{\prime}: C_{\bullet} \rightarrow C_{\bullet}$ be the projection onto $C_{\bullet}^{\prime}$ associated with this projection. This is an $S$-map, since $C_{\bullet}=C_{\bullet}^{\prime} \oplus R_{\bullet}^{T}$ is a splitting of para- $S$-modules. This splitting also implies that the canonical projection $\pi_{T}: C \bullet \rightarrow C_{T}$ • induces a $k$-module isomorphism $\pi_{T}^{\prime}: C_{\bullet}^{\prime} \rightarrow C_{T, \bullet}$. This is an $S$-map, and so we get an $S$-module isomorphism from $C^{\prime}$ onto $C_{T}$. Under this isomorphism the inclusion of $C_{\bullet}^{\prime}$ to $C_{\bullet}$ corresponds to the $S$-map $\iota_{T}^{\prime}: C_{T, \bullet} \rightarrow C$ • given by

$$
\iota_{T}^{\prime}(\bar{x})=\iota_{T}^{\prime}\left(\overline{\pi^{\prime}(x)}\right)=\pi^{\prime}(x), \quad x \in C_{\bullet} .
$$

This means that $\iota_{T}^{\prime} \pi_{T}=\pi^{\prime}$. This gives $\pi_{T} \iota_{T}^{\prime} \pi_{T}=\pi_{T} \pi^{\prime}=\pi_{T}$, and hence $\pi_{T} \iota_{T}^{\prime}=1$ on $C_{T, \bullet}$.

By assumption $R^{T}$ is $S$-contractible, and so there is an $(S, T)$-compatible $k$-linear map $\beta$ : $R_{\bullet}^{T} \rightarrow R_{\bullet+1}^{T}$ such that $1=d \beta+\beta d$. Let $h: C \bullet \rightarrow C_{\bullet+1}$ be the $k$-linear map defined by

$$
h(x)=-\beta\left(x-\pi^{\prime}(x)\right), \quad x \in C_{\bullet} .
$$

This map is compatible with the operators $S$ and $T$. Moreover, as $\pi^{\prime}$ is an $S$-map, given any $x \in C$, we see that $d h(x)+h(d x)$ is equal to

$$
-d \beta\left(x-\pi^{\prime}(x)\right)-\beta\left(d x-\pi^{\prime}(d x)\right)=-(d \beta+\beta d)\left(x-\pi^{\prime}(x)\right)=-\left(x-\pi^{\prime}(x)\right) .
$$

It then follows that $1+d h+h d=\pi^{\prime}=\iota_{T}^{\prime} \pi_{T}$. Together with the equality $\pi_{T} \iota_{T}^{\prime}=1$ above this shows that we have an $S$-deformation retract of $C$ to $C_{T}$. The proof is complete.

Remark 2.13. As we can see from (2.7), the range of $h$ is contained in $R_{\bullet}^{T}=\operatorname{ker} \pi_{T}$ and its kernel contains on $C_{\bullet}^{\prime}=\operatorname{ran} \iota_{T}^{\prime}$. It then follows that $\pi_{T} h=0$ and $h \iota_{T}^{\prime}=0$.

Proposition 2.12 is the main impetus for introducing the following class of para- $S$-modules.

Definition 2.14. A quasi-S-module is a para-S-module $C=\left(C_{\bullet}^{T}, d, S\right)$ such that $R^{T}$ is $S$ contractible and we have the $k$-module splitting,

$$
C_{\bullet}=C_{\bullet}^{T} \oplus R_{\bullet}^{T} .
$$

Remark 2.15. As we shall see, in many instances the $S$-contractibility of $R^{T}$ is ensured by the sole splitting (2.8).

When it occurs the splitting (2.8) is a splitting of para-S-modules. In such a case we shall let $\pi^{T}: C_{\bullet} \rightarrow C_{\bullet}$ be the projection on $C_{T}$ associated with this splitting, and, given any $x \in C_{\bullet}$, we set $x^{T}=\pi^{T}(x)$. 
Lemma 2.16. Suppose there is a polynomial $Q(X) \in k[X]$ such that $Q(T)(T-1)=0$ and $Q(1)=1$. Then $C$ is a quasi-S-module and we have $\pi^{T}=Q(T)$.

Proof. The assumption that $Q(T)(1-T)=(1-T) Q(T)=0$ ensures us that $R_{\bullet}^{T} \subset \operatorname{ker} Q(T)$ and $\operatorname{ran} Q(T) \subset C_{\bullet}^{T}$. The fact that $Q(T) T=Q(T)$ implies that $Q(T) T^{j}=Q(T)$ for all $j \geqslant 0$, and so $Q(T) P(T)=Q(T) P(T)=P(1) Q(T)$ for every polynomial $P(X) \in k[X]$. In particular, taking $P(X)=Q(X)$ gives $Q(T)^{2}=Q(1) Q(T)=Q(T)$. Thus, $Q(T)$ is an idempotent, and so we have the direct sum of $k$-modules,

$$
C_{\bullet}=\operatorname{ran} Q(T) \oplus \operatorname{ker} Q(T) .
$$

To complete the proof we just need to show that $\operatorname{ker} Q(T) \subset R_{\bullet}^{T}$ and $C_{\bullet}^{T} \subset \operatorname{ran} Q(T)$. As $Q(1)=1$ there is $\tilde{Q}(X) \in k[X]$ such that $Q(X)-1=(X-1) \tilde{Q}(X)$. If $x \in \operatorname{ker} Q(T)$, then we have $x=(1-Q(T)) x=(1-T) \tilde{Q}(T) x \in \operatorname{ran}(1-T)$. Thus, $\operatorname{ker} Q(T) \subset R_{\bullet}^{T}$. In addition, if $x \in C_{\bullet}^{T}$, then $Q(T) x-x=\tilde{Q}(T)(T-1) x=0$, and so $x=Q(T) x \in \operatorname{ran} Q(T)$. This shows that $C_{\bullet}^{T} \subset \operatorname{ran} Q(T)$. The proof is complete.

Example 2.17. Suppose that $T^{r}=1$ with $r \geqslant 1$ such that $r^{-1} \in k$ (this happens for instance when $k \supset \mathbb{Q})$. Then Lemma 2.16 holds with $Q(X)=r^{-1}\left(1+X+\cdots+X^{r-1}\right)$. In this case, we have

$$
x^{T}=\frac{1}{r}\left(x+T x+\cdots+T^{r-1} x\right) \quad \forall x \in C_{\bullet} .
$$

Let $C=(C \bullet, d, S, T)$ be a quasi- $S$-module. As the conditions (i)-(ii) of Proposition 2.12 are satisfied we know that $C$ has property (DR). Moreover, the proof of Proposition2.12 above provides us with an explicit $S$-deformation retract of $C$ to $C^{T}$ and $C_{T}$ by using taking the projection $\pi^{\prime}$ to be $\pi^{T}$. More precisely, let $\iota_{T}: C_{T, \bullet} \rightarrow C$ • be the $k$-linear map given by

$$
\iota_{T}(\bar{x})=x^{T}, \quad x \in C_{\bullet} .
$$

We also let $\beta: R_{\bullet}^{T} \rightarrow R_{\bullet+1}^{T}$ be an $(S, T)$-compatible contracting homotopy, i.e., $1=d \beta+\beta d$ on $R_{\bullet}^{T}$. We define the $k$-linear map $h: C_{\bullet} \rightarrow C_{\bullet+1}$ defined by

$$
h(x)=-\beta\left(x-x^{T}\right), \quad x \in C_{\bullet} .
$$

Proposition 2.9 and the second part of the proof of Proposition 2.12 then give the following result.

Proposition 2.18. Suppose that $\left(C_{\bullet}, d, S, T\right)$ is a quasi-S-module.

(1) The canonical projection $\pi^{T}: C_{\bullet} \rightarrow C_{T, \bullet}$ induces an $S$-module isomorphism from $C^{T}$ onto $C_{T}$.

(2) The map $\iota_{T}: C_{T, \bullet} \rightarrow C$ • is an $S$-map such that

$$
\pi_{T} \iota_{T}=1, \quad \pi^{T}=\iota_{T} \pi_{T}=1+d h+h d .
$$

This provides us with $S$-deformation retracts of $C$ to $C_{T}$ and $C^{T}$ and $T$-deformation retracts of $C^{\sharp}$ to $C_{T}^{\sharp}$ and $C^{T, \sharp}$.

Remark 2.19. The $S$-deformation retract of $C^{\natural}$ to $C^{T, \natural}$ is simply given by the inclusion $\iota^{T}: C_{\bullet}^{T} \hookrightarrow$ $C_{\bullet}$ and the projection $x \rightarrow x^{T}$ seen as an $S$-map $\tilde{\pi}^{T}: C_{\bullet} \rightarrow C_{\bullet}^{T}$. There are the $S$-maps $\iota_{T}$ and $\pi_{T}$ under the isomorphism $C_{\bullet}^{T} \simeq C_{T, \bullet}$. In fact, it is immediate that $\tilde{\pi}^{T} \iota^{T}=1$ on $C_{\bullet}^{T}$ and by using (2.11) we get $\iota^{T} \tilde{\pi}^{T}=\pi^{T}=1+d h+h d$.

Remark 2.20. As in Remark 2.13 the chain homotopy $h$ is such that $\pi_{T} h=0$ and $\iota_{T} h=0$.

\section{The Para-S-Module of a Parachain Complex}

In this section, we review the construction of the para-S-module of a parachain complex due to Getzler-Jones [21]. Although, Getzler-Jones didn't not consider para-S-modules in their article, their construction actually yields a para- $S$-module. We shall also introduce quasi-mixed complexes as the parachain complexes that give rise to quasi- $S$-modules. 
3.1. The Para-S-module $C^{\natural}$. Let $C=\left(C_{\bullet}, b, B\right)$ be a parachain complex of $k$-modules in the sense of Getzler-Jones [21] (see also [16]). Recall this means that $\left(C_{\bullet}, b\right)$ is a chain complex of $k$-modules and we have an extra degree 1 differential $B: C_{\bullet} \rightarrow C_{\bullet+1}, B^{2}=0$, such that $T:=1-(b B+B b)$ is a $k$-linear isomorphism. When $T=1$ we recover the definition of a mixed complex [6, 33. Even when $T \neq 1$, the map $T$ commutes with the differentials $(b, B)$, and so this is an automorphism of the parachain complex $C$.

A number of parachain complexes arise from paracylic modules, and more generally $H$-unital para-precyclic modules ( $c f$. Section 5). An example of a different kind of parachain complex is the parachain complex of equivariant noncommutative differential forms of a (non-unital) locally convex algebra acted on by a locally compact group (see Voigt [55]).

As it follows from [21 the datum of a parachain complex $C=(C, b, B)$ allows us to construct a para- $S$-module as follows.

Throughout this paper we let $u$ be an indeterminate variable and denote by $k[u]$ the polynomial algebra over $k$ that it generates. We shall regard the tensor product $k[u] \otimes C \bullet$ as a $k[u]$-module. Given any $m, p \geqslant 0$ and $x \in C_{m}$, we denote by $x u^{p}$ the tensor product $u^{p} \otimes x$. We then have

$$
C_{m} \otimes k[u]=\bigoplus_{p \geqslant 0} C_{m} u^{p}=C_{m} \oplus C_{m} u \oplus C_{m} u^{2} \oplus \cdots,
$$

where we have set $C_{m} u^{p}=\left\{x u^{p} ; x \in C_{m}\right\}$. In addition, we let $u^{-1}: C_{\bullet} \otimes k[u] \rightarrow C_{\bullet} \otimes k[u]$ be the $k$-linear map defined by

$$
u^{-1}\left(x u^{0}\right)=0, \quad u^{-1}\left(x u^{p}\right)=x u^{p-1}, \quad p \geqslant 1 .
$$

We also denote by $u^{-j}, j \geqslant 2$, the operator $\left(u^{-1}\right)^{j}$. Note that $\operatorname{ker} u^{-j}=C \cdot u^{0} \oplus \cdots \oplus C \cdot u^{j-1}$.

The para- $S$-module associated with $\left(C_{\bullet}, b, B\right)$ is $C^{\natural}:=\left(C_{\bullet}^{\natural}, b+B u^{-1}, u^{-1}, T\right)$, where the $k$ module of $m$-chains $C_{m}^{\natural}, m \geqslant 0$, is defined by

$$
C_{m}^{\natural}=\bigoplus_{2 p+q=m} C_{q} u^{p}=C_{m} u^{0} \oplus C_{m-2} u \oplus \cdots .
$$

Here $u^{-1}$ maps $C_{m}^{\natural}$ to $C_{m-2} \oplus C_{m-4} u \oplus \cdots=C_{m-2}^{\natural}$. Moreover, we have

$$
\left(b+B u^{-1}\right)^{2}=(b B+B b) u^{-1}=(1-T) u^{-1} .
$$

In particular, when $C$ is a mixed complex, i.e., $T=1$, we recover the usual cyclic complex of a mixed complex [6, 7, 8, 9, 33.

As the $S$-operator $u^{-1}$ simply shifts $\left(x_{m}, x_{m-2}, \ldots\right)$ to $\left(x_{m-2}, x_{m-4}, \ldots\right)$, the inverse limit (2.2) is naturally identified with the direct product,

$$
C_{i}^{\sharp}=\prod_{q \geqslant 0} C_{2 q+i}, \quad i=0,1 .
$$

Namely, the identification between $\varliminf_{u^{-1}} C_{i+2 q}^{\sharp}$ and $\prod_{q \geqslant 0} C_{2 q+i}$ is given by $\left(x_{i+2 q}\right) \rightarrow\left(\pi_{0}^{\natural}\left(x_{i+2 q}\right)\right)$, where $\pi_{0}^{\natural}: C_{\bullet}^{\natural} \rightarrow C_{\bullet}$ is the projection onto the zeroth degree summand $C_{\bullet} u^{0}=C_{\bullet}$. Under this identification, the differential of $C^{\sharp}$ is just $b+B$. Note that $(b+B)^{2}=b B+B b=1-T$.

Definition 3.1. The para-complex $C^{\sharp}:=\left(C_{\bullet}^{\sharp}, b+B, T\right)$ is called the periodic para-complex of the parachain complex $C$.

Given parachain complexes $\left(C_{\bullet}, b, B\right)$ and $\left(\bar{C}_{\bullet}, b, B\right)$, an $S$-map $f: C_{\bullet}^{\natural} \rightarrow \bar{C}_{\bullet}^{\natural}$ is a chain map of the form,

$$
f=f^{(0)}+f^{(1)} u^{-1}+f^{(2)} u^{-2}+\cdots,
$$

where the sum is finite and the $k$-linear maps $f^{(j)}: C_{\bullet} \rightarrow \bar{C}_{\bullet+2 j}, j \geqslant 0$, are compatible with the $T$-operator. The chain map condition means that

$$
b f^{(0)}=f^{(0)} b, \quad b f^{(j)}+B f^{(j-1)}=f^{(j)} b+f^{(j-1)} B, \quad j \geqslant 1 .
$$

For instance, any map of parachain complexes $f: C_{\bullet} \rightarrow \bar{C} \bullet$ is an $S$-map with $f^{(0)}=f$ and $f^{(j)}=0$ for $j \geqslant 1$. When $T=1$ we recover the usual notion of $S$-map between cyclic complexes of mixed complexes in the sense of 33 . 
Note also that (3.3) implies that $f^{(0)}$ is an ordinary chain map from $\left(C_{\bullet}, b\right)$ to $\left(\bar{C}_{\bullet}, b\right)$. In addition, the corresponding chain map $f^{\sharp}: C_{\bullet}^{\sharp} \rightarrow \bar{C}_{\bullet}^{\sharp}$ is given by

$$
f^{\sharp}\left(\left(x_{2 q+i}\right)_{q \geqslant 0}\right)=\left(f^{(0)}\left(x_{2 q+i}\right)+f^{(1)}\left(x_{2 q-2+i}\right)+\cdots+f^{(q)}\left(x_{i}\right)\right)_{q \geqslant 0} .
$$

3.2. Quasi-mixed complexes. We shall now look at parachain complexes $C$ for which the para$S$-modules $C^{\natural}$ are quasi- $S$-modules. Let $C=\left(C_{\bullet}, b, B\right)$ be a parachain complex of $k$-modules. As in (2.6) we form the $k$-modules $C_{\bullet}^{T}=\operatorname{ker}(1-T), R_{\bullet}^{T}=\operatorname{ran}(1-T)$ and $C_{T, \bullet}=C_{\bullet} / R_{\bullet}^{T}$. As the differentials $b$ and $B$ commute with the operator $T=1-(b B+b B)$, they induce differentials on $C_{\bullet}^{T}$ and $R_{\bullet}^{T}$ and descend to differentials on $C_{T, \bullet}$. We thus get parachain complexes $C^{T}:=\left(C_{\bullet}^{T}, b, B\right)$, $R^{T}:=\left(R_{\bullet}^{T}, b, B\right)$, and $C_{T}:=\left(C_{T, \bullet}, b, B\right)$. Furthermore, as $b B+b B=1-T=0$ on $C_{\bullet}^{T}$ and $C_{T, \bullet}$, we see that $C^{T}$ and $C_{T}$ are actually mixed complexes.

Definition 3.2. A parachain complex $C=\left(C_{\bullet}, b, B\right)$ such that $C_{\bullet}=C_{\bullet}^{T} \oplus R_{\bullet}^{T}$ is called a quasimixed complex.

By using Lemma 2.16 we obtain the following criterion.

Lemma 3.3. Suppose there is $Q(X) \in k[X]$ such that $Q(T)(T-1)=0$ and $Q(1)=1$. Then $C$ is a quasi-mixed complex.

Example 3.4. In the same way as in Example 2.17, if $T^{r}=1$ for some $r \geqslant 1$ such that $r^{-1} \in k$, then the assumption of Lemma 3.3 is satisfied with $Q(X)=r^{-1}\left(1+X+\cdots+X^{r-1}\right)$. Therefore, in this case $C$ is a quasi-mixed complex. Examples of such quasi-mixed complexes are provided by the parachain complexes associated with $r$-cyclic modules (see Example 4.7 below).

Assume that $C$ is a quasi-mixed complex. We note that the splitting $C_{\bullet}=C_{\bullet}^{T} \oplus R_{\bullet}^{T}$ is actually a splitting of parachain complexes, and so we get a splitting of para- $S$-modules,

$$
C_{\bullet}^{\natural}=C_{\bullet}^{T, \natural} \oplus R_{\bullet}^{T, \natural} .
$$

We also observe that the splitting $C_{\bullet}=C_{\bullet}^{T} \oplus R_{\bullet}^{T}$ implies that $1-T$ induces on $R_{\bullet}^{T}$ a $k$-linear map which is both one-to-one and onto, and so we get a $k$-linear isomorphism of $R_{\bullet}^{T}$. As it is compatible with the differentiels $(b, B)$ we get a parachain complex automorphism. We then let $\beta: R_{\bullet}^{T} \rightarrow R_{\bullet+1}^{T}$ be the $k$-module defined by

$$
\beta=(1-T)^{-1} B=B(1-T)^{-1} .
$$

Lemma 3.5. On $R_{\bullet}^{T}$ we have

$$
b \beta+\beta b=1, \quad B \beta=\beta B=0 .
$$

Proof. It is immediate that $B \beta=B^{2}(1-T)^{-1}=0$, and likewise $\beta B=0$. Moreover, the equality $b B+b B=1-T$ implies that on $R^{T}$ we have

$$
b \beta+\beta b=(b B+B b)(1-T)^{-1}=(1-T)(1-T)^{-1}=1 .
$$

The proof is complete.

Remark 3.6. The fact that $\beta$ is a contracting homotopy was observed by Hadfield-Krähmer [29, Proposition 2.1] in the case of the parachain complex of a twisted cyclic module.

The above lemma implies that on $R_{\bullet}^{T, \natural}$ we have

$$
\left(b+B u^{-1}\right) \beta+\beta\left(b+B u^{-1}\right)=b \beta+\beta b=1 .
$$

The contracting homotopy $\beta: R_{\bullet}^{T, \natural} \rightarrow R_{\bullet+1}^{T, \natural}$ is compatible with the operators $S$ and $T$. Thus, this is an $S$-homotopy, and so the para-S-module $R^{T}$ is $S$-contractible. Combining this with the splitting (3.4) we then arrive at the following result.

Proposition 3.7. If $C$ is a quasi-mixed complex, then $C^{\natural}$ is a quasi-S-module. 
Proposition 3.7 allows us to apply Proposition 2.18. More precisely, let $\pi_{T}: C_{\bullet} \rightarrow C_{T}$, be the canonical projection of $C_{\bullet}$ onto $C_{T, \bullet}$ and $\pi^{T}: C_{\bullet} \rightarrow C_{\bullet}$ the projection on $C_{\bullet}^{T}$ associated with the splitting $C_{\bullet}=C_{\bullet}^{T} \oplus R_{\bullet}^{T}$. They are both maps of parachain complexes. As above, given any $x \in C_{\text {. }}$, we set $\bar{x}=\pi_{T}(x)$ and $x^{T}=\pi^{T}(x)$. The projection $\pi_{T}$ induces an isomorphism of mixed complexes from $C^{T}$ onto $C_{T}$. Under this isomorphism the inclusion of $C^{T}$ into $C \bullet$ corresponds to the parachain complex embedding $\iota_{T}: C_{T, \bullet} \rightarrow C \bullet$ given by

$$
\iota_{T}(\bar{x})=x^{T}, \quad x \in C_{\bullet} .
$$

In addition, we let $h: C_{\bullet} \rightarrow C_{\bullet+1}$ be the $k$-linear map defined by

$$
h(x)=-B(1-T)^{-1}\left(x-x^{T}\right), \quad x \in C \bullet .
$$

This is a $T$-compatible map. It gives the chain homotopy (2.10) on $C_{\bullet}^{\natural}$ associated with the contracting homotopy $\beta=-B(1-T)^{-1}$ of $R^{T, \natural}$. Therefore, by using Proposition 2.18 we obtain the following result.

Proposition 3.8. Suppose that $C$ is a quasi-mixed complex.

(1) The canonical projection $\pi_{T}: C_{\bullet} \rightarrow C_{T}$ • induces a mixed complex isomorphism from $C^{T}$ onto $C_{T}$.

(2) The map $\iota_{T}: C_{\bullet} \rightarrow C_{\bullet}$ given by (2.9) is a parachain complex embedding.

(3) We have

$$
\pi_{T} \iota_{T}=1, \quad \iota_{T} \pi_{T}=\pi^{T}=1+\left(b+B u^{-1}\right) h+h\left(b+B u^{-1}\right) .
$$

This provides us with $S$-deformation retracts of $C^{\natural}$ to $C_{T}^{\natural}$ and $C^{T, \natural}$ and $T$-deformation retracts of $C^{\sharp}$ to $C_{T}^{\sharp}$ and $C^{T, \sharp}$.

Remark 3.9. In the same way as in Remark 2.13, we have $\pi_{T} h=0$ and $h \iota_{T}=0$. Moreover, for all $x \in C_{\bullet}$, we have

$$
h^{2}(x)=B(1-T)^{-1} B(1-T)^{-1}\left(x-x^{T}\right)=(1-T)^{-1} B^{2}(1-T)^{-1}\left(x-x^{T}\right)=0 .
$$

That is, $h^{2}=0$. Therefore, we see that the chain homotopy $h$ is special in the sense of (7.11).

Finally, when $C$ is a quasi-mixed complex we also can modify the $B$-operator to get an actual mixed complex whose cyclic $S$-module is $S$-homotopy equivalent to $C^{\natural}$. Namely, we have the following result.

Proposition 3.10. Suppose that $C=\left(C_{\bullet}, b, B\right)$ is a quasi-mixed complex and set $\tilde{B}=\pi^{T} B$.

(1) $\tilde{C}:=\left(C_{\bullet}, b, \tilde{B}\right)$ is a mixed complex.

(2) The projection $\pi^{T}: C_{\bullet} \rightarrow C_{\bullet}$ gives rise to an $S$-homotopy equivalence between $C^{\natural}$ and $\tilde{C}^{\natural}$ and a T-homotopy equivalence between their periodic para-complexes.

Proof. As the projection $\pi^{T}$ is a parachain complex map we have $\tilde{B}^{2}=\pi^{T} B^{2}=0$. As the range of $\pi^{T}$ is $C_{\bullet}^{T}=\operatorname{ker}(1-T)$ we also have $b \tilde{B}+\tilde{B} b=(b B+B b) \pi^{T}=(1-T) \pi^{T}=0$. We thus get a mixed complex $\tilde{C}:=(C \bullet, b, \tilde{B})$. Moreover, as $\left[b, \pi_{T}\right]=0$ and $\pi^{T} \tilde{B}=\tilde{B} \pi^{T}=\pi^{T} B=B \pi^{T}$, we see that $\pi^{T}: C \bullet \rightarrow C \bullet$ is a parachain complex map from $C$ (resp., $\tilde{C}$ ) to $\tilde{C}$ (resp., $C$ ).

By Proposition 3.8 we have $\left(\pi^{T}\right)^{2}=\pi^{T}=1+\left(b+B u^{-1}\right) h+h\left(b+B u^{-1}\right)$. In fact, it follows from Lemma 3.5 and the definition of $h$ that $B h=h B=0$. Thus, $\tilde{B} h=\pi^{T} B h=0$ and $h \tilde{B}=h B \pi^{T}=0$, and so we also have $\left(\pi^{T}\right)^{2}=1+\left(b+\tilde{B} u^{-1}\right) h+h\left(b+\tilde{B} u^{-1}\right)$. This shows that the $S$-maps $\pi^{T}: C_{\bullet}^{\natural} \rightarrow \tilde{C}_{\bullet}^{\natural}$ and $S$-maps $\pi^{T}: C_{\bullet}^{\natural} \rightarrow \tilde{C}_{\bullet}^{\natural}$ are $S$-homotopy inverses of each other, and hence $C^{\natural}$ and $\tilde{C}^{\natural}$ are $S$-homotopy equivalent. Furthermore, by using Proposition 2.9 we get a $T$-homotopy equivalence between the periodic para-complexes $C^{\sharp}$ and $\tilde{C}^{\sharp}$. The proof is complete.

\section{Paracyclic and Para-Precyclic Modules}

In this section, we review the main definitions and examples regarding paracyclic and paraprecyclic modules. 
4.1. Paracyclic modules. Recall that a simplicial $k$-module $C=\left(C_{\bullet}, d_{\bullet}, s_{\bullet}\right)$ is given by $k$ modules $C_{m}, m \geqslant 0$, together with $k$-linear maps $d_{j}: C_{m} \rightarrow C_{m+1}, j=0, \ldots, m$, called faces, and $k$-linear maps $s_{j}: C_{m} \rightarrow C_{m+1}, j=0, \ldots, m$, called degeneracies, which satisfy the following relations:

$$
\begin{gathered}
d_{i} d_{j}= \begin{cases}d_{j-1} d_{i}, & i \leqslant j-1, \\
d_{j} d_{i+1} & i \geqslant j,\end{cases} \\
s_{i} s_{j}=\left\{\begin{array}{cl}
s_{j+1} s_{i}, & i \leqslant j, \\
s_{j} s_{i-1}, & i \geqslant j+1,
\end{array}\right. \\
d_{i} s_{j}=\left\{\begin{array}{cc}
s_{j-1} d_{i}, & i \leqslant j-1, \\
1, & i=j, j+1, \\
s_{j} d_{i-1}, & i \geqslant j+2 .
\end{array}\right.
\end{gathered}
$$

A paracyclic $k$-module [19, 21] (see also [16, 18]) is a simplicial $k$-module $\left(C_{\bullet}, d_{\bullet}, s_{\bullet}\right)$ together with an invertible $k$-linear map $t: C \bullet \rightarrow C$ • such that

$$
\begin{aligned}
& \left\{\begin{array}{l}
t d_{i}=d_{i+1} t, \quad 0 \leqslant i \leqslant m-1, \\
d_{m}=d_{0} t,
\end{array}\right. \\
& \left\{\begin{array}{l}
t s_{j}=s_{j+1} t, \quad 0 \leqslant j \leqslant m-1, \\
t s_{m}=s_{-1} .
\end{array}\right.
\end{aligned}
$$

Here $s_{-1}: C_{\bullet} \rightarrow C_{\bullet+1}$ is the extra-degeneracy, i.e.,

$$
s_{-1}:=t^{-1} s_{0} t .
$$

We obtain a cyclic $k$-module in the sense of Connes 8 when $t^{m+1}=1$ on $C_{m}$ for all $m \geqslant 0$.

A paracyclic module structure is uniquely determined by the end-face $d=d_{m}$, the extra degeneracy $s=s_{-1}$ and the paracyclic operator $t=d s$. Indeed, it follows from (4.4) and (4.5) that we have

$$
d_{i}=t^{i-m} d t^{m-i}, \quad s_{i}=t^{i+1} s t^{-(i+1)}, \quad i=0, \ldots, m .
$$

Combining this with the relations $d_{m}=d_{0} t$ and $s_{-1}=t s_{m}$ we further see that $d=d_{0} t=t^{-m} d t^{m+1}$ and $s=t s_{m}=t^{m+2} s t^{-(m+1)}$. Thus, even in the non-cyclic case, the operator $T:=t^{m+1}$ is invertible and commutes with the structural operators $(d, s, t)$. That is, this is a paracylic-module automorphism.

We also record the following relations satisfied by the extra-degeneracy:

$$
\begin{gathered}
s_{-1} s_{j}=s_{j+1} s_{-1}, \\
d_{i} s_{-1}= \begin{cases}1 & i=0 \\
s_{-1} d_{i-1} & 0 \leqslant j \leqslant m-1, \\
t & i=m+1\end{cases}
\end{gathered}
$$

Example $4.1([29,39])$. Let $\mathcal{A}$ be a unital $k$-algebra and $\sigma: \mathcal{A} \rightarrow \mathcal{A}$ a unital algebra automorphism. The corresponding twisted cyclic $k$-module $C^{\sigma}(\mathcal{A})$ is the paracyclic $k$-module $\left(C \bullet(\mathcal{A}), d_{\sigma}, s, t_{\sigma}\right)$, where $C_{m}(\mathcal{A})=\mathcal{A}^{\otimes(m+1)}, m \geqslant 0$, and the structural operators $\left(d_{\sigma}, s, t_{\sigma}\right)$ are given by

$$
\begin{aligned}
d_{\sigma}\left(a^{0} \otimes \cdots \otimes a^{m}\right) & =\left(\sigma\left(a^{m}\right) a^{0}\right) \otimes a^{1} \otimes \cdots \otimes a^{m-1}, \\
s\left(a^{0} \otimes \cdots \otimes a^{m}\right) & =1 \otimes a^{0} \otimes \cdots \otimes a^{m}, \\
t_{\sigma}\left(a^{0} \otimes \cdots \otimes a^{m}\right) & =a^{m} \otimes a^{0} \otimes \cdots \otimes a^{m-1}, \quad a^{j} \in \mathcal{A} .
\end{aligned}
$$

When $\sigma=1$ we recover the usual cyclic module of a unital algebra 8,9 . In general, the operator $T=t_{\sigma}^{m+1}$ is just the $k$-module isomorphism $\sigma^{\otimes(m+1)}$ on $C_{m}(\mathcal{A})=\mathcal{A}^{\otimes(m+1)}$. 
Example 4.2. Let $\Gamma$ be a group and $\phi$ a normal element of $\Gamma$. When $k$ has a unit these data give rise to the paracyclic $k$-module $C^{\phi}(\Gamma)=\left(C \bullet(\Gamma), d, s_{\phi}, t_{\phi}\right)$, where $C_{m}(\Gamma)=k \Gamma^{m+1}, m \geqslant 0$, and the structural operators $\left(d, s_{\phi}, t_{\phi}\right)$ are given by

$$
\begin{aligned}
d\left(\gamma_{0}, \ldots, \gamma_{m}\right) & =\left(\gamma_{0}, \ldots, \gamma_{m-1}\right), \\
s_{\phi}\left(\gamma_{0}, \ldots, \gamma_{m}\right) & =\left(\phi^{-1} \gamma_{m}, \gamma_{0}, \ldots, \gamma_{m}\right), \\
t_{\phi}\left(\gamma_{0}, \ldots, \gamma_{m}\right) & =\left(\phi^{-1} \gamma_{m}, \gamma_{0}, \ldots, \gamma_{m-1}\right), \quad \gamma_{j} \in \Gamma .
\end{aligned}
$$

When $\phi=1$ we recover the standard cyclic $k$-module of $\Gamma$ (see, e.g., 42]). In general, the operator $T=t_{\phi}^{m+1}$ on $C_{m}(\Gamma)$ arises from the diagonal left-action of $\phi^{-1}$ on $\Gamma^{m+1}$. Namely, we have

$$
T\left(\gamma_{0}, \ldots, \gamma_{m}\right)=\left(\phi^{-1} \gamma_{0}, \ldots, \phi^{-1} \gamma_{m}\right), \quad \gamma_{j} \in \Gamma .
$$

In any case, the $b$-differential of $C^{\phi}(\Gamma)$ is always the group differential (2.1).

Remark 4.3. Paracyclic modules also naturally appear in Hopf cyclic theory (see [12, 14, 26, 35]).

As in (2.6) we introduce the $k$-modules $C_{\bullet}^{T}=\operatorname{ker}(1-T), R_{\bullet}^{T}=\operatorname{ran}(1-T)$, and $C_{T, \bullet}=C_{\bullet} / R_{\bullet}^{T}$. As the structural operators $(d, s, t)$ commute with the operator $T$, they induce structural operators on the $k$-modules $C_{\bullet}^{T}$ and $R_{\bullet}^{T}$ and they descend to structural operators on $C_{T, \bullet}$. We thus obtain paracyclic $k$-modules $C^{T}:=\left(C_{\bullet}^{T}, d, s, t\right), R^{T}:=\left(R_{\bullet}^{T}, d, s, t\right)$ and $C_{T}=\left(C_{T, \boldsymbol{\bullet}}, d, s, t\right)$. In addition, as $t^{m+1}=T=1$ on $C_{m}^{T}$ and $C_{T, m}$ we see that $C^{T}$ and $C_{T}$ are both cyclic $k$-modules.

Definition 4.4 (37]). A quasi-cyclic $k$-module is a paracyclic $k$-module $C=(C, d, s, t)$ such that $C_{\bullet}=C_{\bullet}^{T} \oplus R_{\bullet}^{T}$.

Remark 4.5. If $C$ is a quasi-cyclic $k$-module, then the splitting $C_{\bullet}=C_{\bullet}^{T} \oplus R_{\bullet}^{T}$ is a splitting of paracyclic $k$-modules.

In the same way as with Lemma 3.3 we have the following result.

Lemma 4.6. Suppose that $C=(C \bullet, d, s, t)$ is a paracyclic $k$-module for which there is a polynomial $Q(X) \in k[X]$ such that $Q(T)(T-1)=0$ and $Q(1)=1$. Then $C$ is a quasi-cyclic $k$-module.

Example 4.7 (Feigin-Tsygan [19]; see also [4). In the terminology of [19, given $r \geqslant 1$, an $r$-cyclic $k$-module is a paracyclic $k$-module $C=(C \bullet, d, s, t)$ for which $T^{r}=1$. In the same way as in Example 2.17, when $r^{-1} \in k$ the assumptions of Lemma 4.6 are satisfied, and so in this case any $r$-cyclic $k$-module is a quasi-cyclic $k$-module. Instances of $r$-cyclic $k$-modules include the following:

- The twisted cyclic $k$-module $C^{\sigma}(\mathcal{A})$ of Example 4.1 when the automorphism $\sigma$ is such that $\sigma^{r}=1$.

- The paracyclic $k$-module $C^{\phi}(\Gamma)$ of Example 4.2, when $\phi$ has order $r$.

Remark 4.8. We refer to [25, Example 3.10] for an example of a paracyclic module which is not a quasi-cyclic module.

4.2. Para-precylic Modules. Recall that a pre-simplicial $k$-module $C=\left(C_{\bullet}, d_{\bullet}\right)$ is given by $k$-modules $C_{m}, m \geqslant 0$, and $k$-linear maps $d_{j}: C_{m} \rightarrow C_{m+1}, j=0, \ldots, m$, satisfying the face relations (4.1). In this paper, we shall use the following definition of a para-precyclic $k$-module.

Definition 4.9 (46). A para-precyclic $k$-module $C=\left(C_{\bullet}, d_{\bullet}, t\right)$ is a pre-simplicial $k$-module $\left(C_{\bullet}, d_{\bullet}\right)$ together with an invertible $k$-linear map $t: C_{\bullet} \rightarrow C_{\bullet}$ satisfying the relations (4.4).

Remark 4.10. Para-precyclic modules are also called quasi-cyclic modules in [46].

When the paracyclic operator $t$ further satisfies the cyclic condition $t^{m+1}=1$ on $C_{m}$ we recover the definition of a precyclic $k$-module [42. In the same way as with a paracyclic module structure, a para-precyclic module structure is uniquely determined by the end face $d=d_{m}$ on $C_{m}$ and the paracyclic operator $t$. Moreover, the operator $T=t_{m}^{m+1}$ commutes with the structural operators $(d, t)$, and so this a para-precyclic module automorphism. 
Example 4.11. Let $\mathcal{A}$ be a $k$-algebra and $\sigma: \mathcal{A} \rightarrow \mathcal{A}$ an algebra automorphism. In the unital case we can form the twisted cyclic module $C^{\sigma}(\mathcal{A})$ of Example 4.1. In general, we can form the paraprecyclic $k$-module $C^{\sigma}(\mathcal{A})=\left(C \bullet(\mathcal{A}), d_{\sigma}, t_{\sigma}\right)$, where $C_{m}=\mathcal{A}^{\otimes(m+1)}, m \geqslant 0$, and the structural operators $\left(d_{\sigma}, t_{\sigma}\right)$ are defined as in (4.8) and (4.10). We obtain the usual precyclic module of an algebra when $\sigma=1$ (see, e.g., 42]).

Let $C=\left(C_{\bullet}, d, t\right)$ be a para-precyclic $k$-module. In the same way as with paracyclic $k$-modules, the fact that the structural operators $(d, t)$ commute with $T$ ensures us that they define a preparacylic $k$-module structure on $R_{\bullet}^{T}=\operatorname{ran}(1-T)$ and precyclic $k$-module structures on $C_{\bullet}^{T}=$ $\operatorname{ker}(1-T)$ and $C_{T, \bullet}=C_{\bullet} / R_{\bullet}^{T}$. We denote by $R^{T}, C^{T}$ and $C_{T}$ the corresponding pre-(para)cyclic $k$-modules.

Definition 4.12. A quasi-precyclic $k$-module is a para-precyclic $k$-module $C=(C \bullet, d, t)$ such that $C_{\bullet}=C_{\bullet}^{T} \oplus R_{\bullet}^{T}$.

Remark 4.13. If $C$ is a quasi-precyclic $k$-module, then the splitting $C_{\bullet}=C_{\bullet}^{T} \oplus R_{\bullet}^{T}$ is a splitting of para-precyclic $k$-modules.

We have the following version of Lemma 4.6 .

Lemma 4.14. Suppose that $C=\left(C_{\bullet}, d, t\right)$ is a para-precyclic $k$-module for which there is a polynomial $Q(X) \in k[X]$ such that $Q(T)(T-1)=0$ and $Q(1)=1$. Then $C$ is a quasi-precyclic $k$-module.

Example 4.15. Given $r \geqslant 1$, an $r$-precyclic $k$-module is a paracyclic $k$-module $C=(C$, $d, t)$ for which $T^{r}=1$. In the same way as in Example 4.7, any $r$-precyclic $k$-module is a quasi-precyclic $k$-module when $r^{-1} \in k$. For instance, the twisted precyclic $k$-module $C^{\sigma}(\mathcal{A})$ of Example 4.11 is $r$-precyclic when the automorphism $\sigma$ is such that $\sigma^{r}=1$.

\section{The Para- $S$-Module of an $H$-Unital Para-Precyclic Module}

In this section, by elaborating on 21 we explain how to associate a parachain complex with any paracyclic module and, more generally, with any $H$-unital para-precyclic module. As a result, this allows us to associate a para- $S$-module with any $H$-unital para-precyclic module. We shall also exhibit some of the properties of these para- $S$-modules.

Any cyclic $k$-module gives rise to a mixed complex [7, 8, 9]. In the terminology of [7, 8, 9 ] the corresponding $C^{\natural}$-complex is precisely the total complex of Connes' $(b, B)$-bicomplex. As it turns out, this construction can be extended to precyclic modules that are $H$-unital, i.e., precyclic modules whose bar complexes are contractible (see, e.g., [34, 57]).

Getzler-Jones 21] observed that the construction of the mixed complex of a cyclic module can be carried out mutatis mutandis to associate a parachain complex with any paracyclic module. In fact, in the paracyclic setting, we only need to be a bit careful with the choice of the contracting homotopy for the bar complex. Moreover, as we shall explain, this construction holds in greater generality for any $H$-unital para-precyclic module.

Let $C=\left(C_{\bullet}, d, s, t\right)$ be a paracyclic $k$-module. As $C$ is a simplicial $k$-module we have differentials $b: C_{\bullet} \rightarrow C_{\bullet-1}$ and $b^{\prime}: C_{\bullet} \rightarrow C_{\bullet-1}$ given by

$$
b=\sum_{0 \leqslant j \leqslant m}(-1)^{j} d_{j} \quad \text { and } \quad b^{\prime}=\sum_{0 \leqslant j \leqslant m-1}(-1)^{j} d_{j} \quad \text { on } C_{m} .
$$

The relations (4.7) imply that the bar complex $\left(C_{\bullet}, b^{\prime}\right)$ is acyclic and a contracting homotopy is provided by the extra degeneracy $s$, i.e., we have

$$
b^{\prime} s+s b^{\prime}=1 \quad \text { on } C_{\bullet} .
$$

Moreover, the extra degeneracy $s$ is compatible with the isomorphism $T=\tau^{m+1}$.

The chain complexes $\left(C_{\bullet}, b\right)$ and $\left(C_{\bullet}, b^{\prime}\right)$ make sense for any pre-simplicial $k$-module. In particular, they make sense for any para-precyclic $k$-module. However, for a general para-precyclic $k$-module, the bar complex $\left(C_{\bullet}, b^{\prime}\right)$ need not be contractible. 
Definition 5.1. A para-precyclic $k$-module $C=\left(C_{\bullet}, d, t\right)$ is called $H$-unital when its bar complex $\left(C \bullet, b^{\prime}\right)$ admits a $T$-compatible contracting homotopy.

An $H$-unital paracyclic $k$-module is thus given by a system $\left(C_{\bullet}, d, s, t\right)$, where $(d, t)$ defines a para-precyclic $k$-module structure on $C$. and $s$ is a $T$-contractible homotopy satisfying (5.2). In particular, any paracyclic $k$-module is an $H$-unital para-precyclic $k$-module.

Example 5.2. At least when $k$ is a division ring, for any $k$-algebra with a local unit the precyclic $k$-module $C(\mathcal{A})$ is $H$-unital (see [42, [57]). As a result, given any automorphism $\sigma: A \rightarrow A$ of finite order, the corresponding twisted precyclic $k$-module $C^{\sigma}(A)$ of Example 4.11 is $H$-unital.

Let $C=\left(C_{\bullet}, d, t\right)$ be a para-precyclic $k$-module. The $k$-linear maps $\tau: C_{\bullet} \rightarrow C_{\bullet}$ and $N: C_{\bullet} \rightarrow$ $C$. are defined by

$$
\tau=(-1)^{m} t \quad \text { and } \quad N=1+\tau+\cdots+\tau^{m} \quad \text { on } C_{m} .
$$

Note that

$$
(1-\tau) N=N(1-\tau)=1-T .
$$

Moreover, in the same way as with cyclic modules [8, 9, 54] (see also [42]) we have

$$
b(1-\tau)=(1-\tau) b^{\prime} \quad \text { and } \quad N b=b^{\prime} N .
$$

Suppose now that we have a contracting homotopy $s^{\prime}: C_{\bullet} \rightarrow C_{\bullet+1}$ satisfying (5.2). The $k$-linear map $B: C \bullet \rightarrow C_{\bullet+1}$ is defined by

$$
B=(1-\tau) s^{\prime} N
$$

When $C$ is cyclic and $s^{\prime}$ is the extra degeneracy we recover the usual $B$-operator [7, 8, 9]. Using (5.4) and the fact that $b^{\prime} s^{\prime}+s^{\prime} b^{\prime}=1$ we see that $b B+B b$ is equal to

$$
b(1-\tau) s^{\prime} N+(1-\tau) s^{\prime} N b=(1-\tau)\left(b^{\prime} s^{\prime}+s^{\prime} b^{\prime}\right) N=(1-\tau) N=1-T .
$$

We also have

$$
B^{2}=(1-\tau) s^{\prime} N(1-\tau) s^{\prime} N=(1-\tau) s^{\prime}(1-T) s^{\prime} N .
$$

Therefore, we arrive at the following result.

Proposition 5.3 (see also [21, 30]). If the contracting homotopy $s^{\prime}$ is such that $s^{\prime}(1-T) s^{\prime}=0$, then $(C \cdot, b, B)$ is a parachain complex such that $b B+B b=1-T$.

Remark 5.4. When $C$ is a cyclic module, i.e., $T=1$, the condition $s^{\prime}(1-T) s^{\prime}=0$ is always satisfied, and so we can choose any contracting homotopy to define the operator $B$. In particular, we may take $s^{\prime}$ to be the extra degeneracy $s$, in which case we recover the usual mixed complex of a cyclic module [8, 9].

Let $C=\left(C_{\bullet}, d, s, t\right)$ be an $H$-unital pre-paracylic $k$-module. If $T \neq 1$, then $s(1-T) s=(1-T) s^{2}$ need not be zero (there seems to be an oversight of this fact in 21]; see also [30 on this point). However, we can replace $s$ by the $k$-linear map $s^{\prime}: C \bullet \rightarrow C \cdot+1$ given by

$$
s^{\prime}=s b^{\prime} s
$$

This is a contracting homotopy, since we have

$$
b^{\prime} s^{\prime}+s^{\prime} b^{\prime}=b^{\prime} s b^{\prime} s+s b^{\prime} s b^{\prime}=\left(1-s b^{\prime}\right) b^{\prime} s+s b^{\prime}\left(1-b^{\prime} s\right)=b^{\prime} s+s b^{\prime}=1 .
$$

In addition, we have

$$
\left(s^{\prime}\right)^{2}=s b^{\prime} s s b^{\prime} s=s\left(1-s b^{\prime}\right)\left(1-b^{\prime} s\right) s=s\left(1-b^{\prime} s-s b^{\prime}\right) s=0 .
$$

As $T$ commutes with $s$ and $b^{\prime}$, and hence with $s^{\prime}$, we deduce that $s^{\prime}(1-T) s^{\prime}=(1-T)\left(s^{\prime}\right)^{2}=0$. Therefore, by using $s^{\prime}=s b^{\prime} s$ to define the operator $B$ we obtain a parachain complex.

Definition 5.5 (compare 21]). Given any $H$-unital para-precyclic $k$-module $C=(C \bullet, d, s, t)$ its parachain complex is $\left(C_{\bullet}, b, B\right)$, where $b$ is defined as in (5.1) and $B$ is given by (5.5) with $s^{\prime}:=s b^{\prime} s$. The corresponding para-complex $C^{\sharp}:=\left(C_{\bullet}^{\sharp}, b+B, T\right)$ is called the periodic para-complex of $C$. 
Remark 5.6. When $C$ is a cyclic $k$-module, we get a mixed complex which is different from the usual mixed complex of a cyclic $k$-module, since for the latter the $B$-differential is defined by using the extra degeneracy $s$ instead of the special homotopy $s^{\prime}=b^{\prime} s b^{\prime}$. As it turns out, the two mixed complexes are isomorphic (see Proposition 5.9 below).

Let $C=\left(C_{\bullet}, d, s, t\right)$ be an $H$-unital quasi-precylic $k$-module, so that $C_{\bullet}=C_{\bullet}^{T} \oplus R_{\bullet}^{T}$. This implies that the parachain complex $(C, b, B)$ is a quasi-mixed complex. As the contracting homotopy $s$ is compatible with the $T$-operator, it induces contracting homotopies for the bar complexes of $C^{T}$ and $R^{T}$ and it descends to a contracting homotopy for the bar complex of $C_{T}$. Thus, the para-precyclic $k$-module $R^{T}$ and the precyclic $k$-modules $C^{T}$ and $C_{T}$ are all $H$-unital. Moreover, their parachain complexes are precisely the parachain complexes $C^{T}, R^{T}, C_{T}$ associated with the parachain complex $\left(C_{\bullet}, b, B\right)$. In addition, the canonical projection $\pi_{T}: C_{\bullet} \rightarrow C_{T}^{\bullet}$ and the projection $\pi^{T}: C_{\bullet} \rightarrow C_{\bullet}$ on $C^{T}$ defined by the splitting $C_{\bullet}=C_{\bullet}^{T} \oplus R_{\bullet}^{T}$ are both maps of $H$-unital para-precyclic $k$-modules. Therefore, by using Proposition 3.7 we arrive at the following result.

Proposition 5.7 (see also [29, Proposition 2.1]). Assume that $C$ is a quasi-cyclic (resp., H-unital quasi-precyclic) $k$-module.

(1) The canonical projection $\pi_{T}: C_{\bullet} \rightarrow C_{T,}$ induces an isomorphism of cyclic (resp., precyclic) k-modules from $C^{T}$ onto $C_{T}$.

(2) The embedding $\iota_{T}: C_{T, \bullet} \rightarrow C_{\bullet}^{T}$ given by (2.9) is an embedding of paracyclic (resp., paraprecyclic) $k$-modules.

(3) We have

$$
\pi_{T} \iota_{T}=1, \quad \iota_{T} \pi_{T}=\pi^{T}=1+\left(b+B u^{-1}\right) h+h\left(b+B u^{-1}\right) .
$$

This provides us with $S$-deformation retracts of $C^{\natural}$ to $C_{T}^{\natural}$ and $C^{T, \natural}$, and with $T$-deformation retracts of $C^{\sharp}$ to $C_{T}^{\sharp}$ and $C^{T, \sharp}$.

As $(C, b, B)$ is a quasi-mixed complex, by Proposition 3.10 we also have a mixed complex $\tilde{C}=\left(C_{\bullet}, b, \tilde{B}\right)$ whose $S$-module is $S$-homotopy equivalent to $C^{\natural}$. Here $\tilde{B}=B \pi^{T}=(1-\tau) s^{\prime} N \pi^{T}$, and so $\tilde{C}$ is defined in the same way as $(C \bullet, b, B)$, upon replacing $N$ by $\tilde{N}:=N \pi^{T}$ in the formula (5.5) for $B$.

When $C$ is an $r$-precyclic $k$-module and $r^{-1} \in k$, we know by Lemma 3.3 that $C$ is quasi-precyclic and $\pi^{T}=r^{-1}\left(1+\cdots+T^{r-1}\right)$. On $C_{m}$ we then have

$$
r \tilde{N}=\frac{1}{r} N\left(1+\cdots+T^{r-1}\right)=\left(\sum_{0 \leqslant j \leqslant m} \tau^{j}\right)\left(\sum_{0 \leqslant l \leqslant r-1} \tau^{(m+1) l}\right)=\sum_{j=0}^{r(m+1)-1} \tau^{j} .
$$

Thus, $r \tilde{N}$ agrees with the $N$-operator for $r$-cyclic $k$-modules of Feigin-Tsygan [19, Appendix].

5.1. Dependence on the contracting homotopy $s^{\prime}$. As it turns out, the choice of the contracting homotopy $s^{\prime}$ is hardly important, since, as we are going to see, all the para- $S$-modules that we get are isomorphic. More precisely, let $C=(C \bullet, d, s, t)$ be an $H$-unital pre-paracylic $k$-module and suppose we are given another contracting homotopy $\hat{s}: C_{\bullet} \rightarrow C_{\bullet+1}$ such that $\hat{s}(1-T) \hat{s}=0$. (In particular, when $C$ a cyclic module we may take $\hat{s}$ to be the extra degeneracy $s$.) We thus get another para-S-module $\left(C^{\natural}, b+\hat{B} u^{-1}, u^{-1}, T\right)$, where $\hat{B}=(1-\tau) \hat{s} N$. Let $f: C_{\bullet}^{\natural} \rightarrow C_{\bullet}^{\natural}$ be the $k$-linear map defined by

$$
f=1+(1-\tau) \hat{s} s^{\prime} N u^{-1} .
$$

This maps commutes with $u^{-1}$ and with the operator $T$. This is also a graded $k$-linear isomorphism with inverse,

$$
f^{-1}=1+\sum_{j \geqslant 1}\left[(1-\tau) \hat{s} s^{\prime} N\right]^{j} u^{-j}
$$

Lemma 5.8. We have

$$
f\left(b+B u^{-1}\right)=\left(b+\hat{B} u^{-1}\right) f .
$$


Proof. The operator $f\left(b+B u^{-1}\right)$ is equal to

$$
\left(1+(1-\tau) \hat{s} s^{\prime} N u^{-1}\right)\left(b+B u^{-1}\right)=b+\left[B+(1-\tau) \hat{s} s^{\prime} N b\right] u^{-1}+(1-\tau) \hat{s} s^{\prime} N B u^{-2} .
$$

By using the relation $N b=b^{\prime} N$ we get

$$
B+(1-\tau) \hat{s} s^{\prime} N b=(1-\tau) s^{\prime} N+(1-\tau) \hat{s} s^{\prime} b^{\prime} N=(1-\tau)\left(s^{\prime}+\hat{s} s^{\prime} b^{\prime}\right) N .
$$

As $s^{\prime}(1-T) s^{\prime}=0$ we also obtain

$$
(1-\tau) \hat{s} s^{\prime} N B=(1-\tau) \hat{s} s^{\prime} N(1-\tau) s^{\prime} N=(1-\tau) \hat{s} s^{\prime}(1-T) s^{\prime} N=0 .
$$

Thus,

$$
f\left(b+B u^{-1}\right)=b+(1-\tau)\left(s^{\prime}+\hat{s} s^{\prime} b^{\prime}\right) N u^{-1} .
$$

Similarly, the operator $\left(b+\hat{B} u^{-1}\right) f$ is equal to

$$
\left(b+\hat{B} u^{-1}\right)\left(1+(1-\tau) \hat{s} s^{\prime} N u^{-1}\right)=b+\left[b(1-\tau) \hat{s} s^{\prime} N+\hat{B}\right] u^{-1}+\hat{B}(1-\tau) \hat{s} s^{\prime} N u^{-2} .
$$

Thanks to the relation $b(1-\tau)=b^{\prime}(1-\tau)$ we obtain

$$
b(1-\tau) \hat{s} s^{\prime} N+\hat{B}=(1-\tau) b^{\prime} \hat{s} s^{\prime} N+(1-\tau) \hat{s} N=(1-\tau)\left[b^{\prime} \hat{s} s^{\prime}+\hat{s}\right] N .
$$

Using the fact that $\hat{s}(1-T) \hat{s}=0$ we also get

$$
\hat{B}(1-\tau) \hat{s} s^{\prime} N=(1-\tau) \hat{s} N(1-\tau) \hat{s} s^{\prime} N=(1-\tau) \hat{s}(1-T) \hat{s} s^{\prime} N=0 .
$$

Thus,

$$
\left(b+\hat{B} u^{-1}\right) f=b+(1-\tau)\left[b^{\prime} \hat{s} s^{\prime}+\hat{s}\right] N u^{-1} .
$$

Combining (5.8) and (5.9) gives

$$
f\left(b+B u^{-1}\right)-\left(b+\hat{B} u^{-1}\right) f=(1-\tau)\left[s^{\prime}-\hat{s}+\hat{s} s^{\prime} b^{\prime}-b^{\prime} \hat{s} s^{\prime}\right] N u^{-1} .
$$

As $\hat{s} s^{\prime} b^{\prime}-b^{\prime} \hat{s} s^{\prime}=\hat{s}\left(1-b^{\prime} s^{\prime}\right)-\left(1-\hat{s} b^{\prime}\right) s^{\prime}=\hat{s}-s^{\prime}$, we deduce that $f\left(b+B u^{-1}\right)-\left(b+\hat{B} u^{-1}\right) f=0$. This proves the result.

Lemma 5.8 asserts that $f$ is a chain map from $\left(C_{\bullet}^{\natural}, b+B u^{-1}\right)$ to $\left(C_{\bullet}^{\natural}, b+\hat{B} u^{-1}\right)$. As this is an isomorphism which commutes with $u^{-1}$ and $T$, we deduce that this is an isomorphism of para- $S$-modules. Therefore, we have proved the following result.

Proposition 5.9. The para-S-modules $\left(C_{\bullet}^{\natural}, b+B u^{-1}, u^{-1}, T\right)$ and $\left(C_{\bullet}^{\natural}, b+\hat{B} u^{-1}, u^{-1}, T\right)$ are isomorphic. An explicit isomorphism is provided by (5.7).

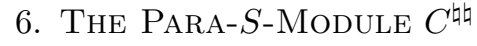

An alternative way to define the cyclic homology of a cyclic $k$-module is to use the $C C$ bicomplex [8, 54. From this point of view the cyclic homology is given by the homology of the total complex of this bicomplex. In fact, this bicomplex makes sense for any precyclic $k$-module, including precyclic modules of non-unital algebras.

In this section, we shall extend this approach to the para-(pre)cyclic setting. Although we will not get a bicomplex, we will still be able to construct a para- $S$-module. We shall work in the setting of para-precyclic $k$-modules. 


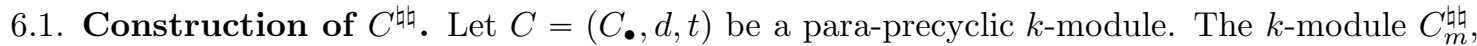
$m \geqslant 0$, is defined by

$$
C_{m}^{\text {घh }}=\bigoplus_{p+q=m} C_{q} u^{p}=C_{m} u^{0} \oplus C_{m-1} u \oplus C_{m-2} u^{2} \oplus \cdots .
$$

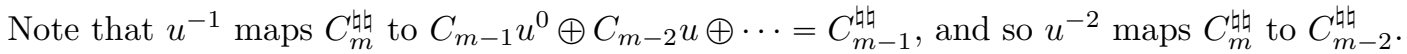

We define the operator $\partial: C_{\bullet}^{\text {蚂 }} \rightarrow C_{\bullet-1}^{\text {蛄 }}$ by

$$
\partial\left(x u^{2 p+1}\right)=(1-\tau) x u^{2 p}, \quad \partial\left(x u^{2 p}\right)=\left\{\begin{array}{cc}
0 & \text { if } p=0, \\
N x u^{2 p-1} & \text { if } p \geqslant 1,
\end{array}\right.
$$

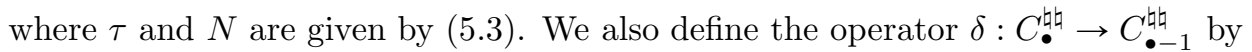

$$
\delta\left(x u^{2 p}\right)=b x u^{2 p}, \quad \delta\left(x u^{2 p+1}\right)=-b^{\prime} x u^{2 p+1} .
$$

Lemma 6.1 (see also [8, 43, 54]). We have

$$
\partial^{2}=(1-T) u^{-2} \quad \text { and } \quad \partial \delta+\delta \partial=\delta^{2}=0 .
$$

Proof. It is immediate that $\delta^{2}=0$, since $b^{2}=\left(b^{\prime}\right)^{2}=0$. Let $x \in C_{\bullet}$. Note that $\partial=N u^{-1}$ on $C \cdot u^{2 p}$ and $\partial=(1-t) u^{-1}$ on $C \cdot u^{2 p+1}$. Thus,

$$
\partial^{2}\left(x u^{2 p}\right)=\partial N u^{-1}\left(x u^{2 p}\right)=(1-\tau) N u^{-2}\left(x u^{2 p}\right)=(1-T) u^{-2}\left(x u^{2 p}\right) .
$$

Likewise, we have

$$
\partial^{2}\left(x u^{2 p+1}\right)=\partial(1-\tau) u^{-1}\left(x u^{2 p+1}\right)=N(1-\tau) u^{-2}\left(x u^{2 p+1}\right)=(1-T) u^{-2}\left(x u^{2 p+1}\right) .
$$

Therefore, we see that $\partial^{2}=(1-T) u^{-2}$.

We have $(\partial \delta+\delta \partial)\left(x u^{0}\right)=\partial\left(b x u^{0}\right)=0$. If $p \geqslant 1$, then by using the relation $N b=b^{\prime} N$ we get

$$
(\partial \delta+\delta \partial)\left(x u^{2 p}\right)=\partial\left(b x u^{2 p}\right)+\delta\left(N x u^{2 p-1}\right)=\left(N b x-b^{\prime} N x\right) u^{2 p-1}=0 .
$$

If $p \geqslant 0$, then the equality $b(1-\tau)=(1-\tau) b^{\prime}$ implies that we have

$$
(\partial \delta+\delta \partial)\left(x u^{2 p}\right)=-\partial\left(b^{\prime} x u^{2 p+1}\right)+\delta\left((1-\tau) x u^{2 p}\right)=\left[-b^{\prime}(1-\tau) x+b(1-\tau) x\right] u^{2 p}=0 .
$$

It then follows that $\partial \delta+\delta \partial=0$. The proof is complete.

It follows from Lemma 6.1 that we have

$$
(\partial+\delta)^{2}=\partial^{2}+\partial \delta+\delta \partial+\delta^{2}=(1-T) u^{-2} .
$$

As $u^{-1}$ and $T$ both commute with $\partial$ and $\delta$ we arrive at the following result.

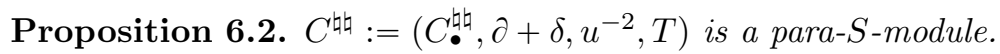

Remark 6.3. If $C$ is a cyclic $k$-module, i.e., $T=1$, then $\partial^{2}=0$ and $(\partial+\delta)^{2}=0$, and so $C^{\text {th }}$ is an $S$-module. In particular, $\left(C_{\bullet}^{\text {घh }}, \partial+\delta\right)$ is a chain complex. In fact, this is the total complex of the bicomplex $\left(C C_{\bullet}, \bullet, \partial, \hat{\delta}\right)$, where

$$
C C_{p, q}=C_{q} u^{p}, \quad \hat{\delta}\left(x u^{2 p}\right)=b x u^{2 p}, \quad \hat{\delta}\left(x u^{2 p+1}\right)=b^{\prime} x u^{2 p+1} .
$$

This bicomplex is precisely the $C C$-bicomplex.

Remark 6.4. When $C$ is an $H$-unital paracyclic $k$-module we obtain two para- $S$-modules $C^{\natural}$ and

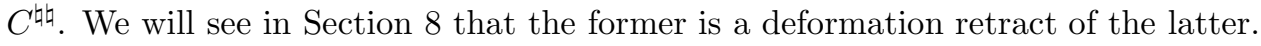


6.2. Quasi-precyclic modules. Supposed now that $C=\left(C_{\bullet}, d, t\right)$ is a quasi-precyclic $k$-module in the sense of Definition 4.12 As mentioned in Remark 4.13 the splitting $C_{\bullet}=C_{\bullet}^{T} \oplus R_{\bullet}^{T}$ is a splitting of para-precyclic $k$-modules. In particular, we get a splitting of para- $S$-modules,

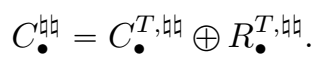

As mentioned in Section 3 the splitting $C_{\bullet}=C_{\bullet}^{T} \oplus R_{\bullet}^{T}$ also ensures us that $1-T$ induces an

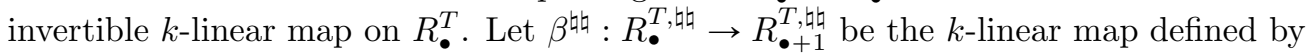

$$
\beta^{\text {घh }}\left(x u^{2 p}\right)=N(1-T)^{-1} x u^{2 p+1}, \quad \beta^{\text {घh }}\left(x u^{2 p+1}\right)=0, \quad x \in R_{\bullet}^{T} .
$$

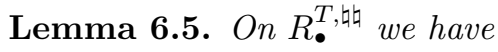

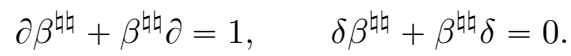

Proof. Let $x \in R_{\bullet}^{T}$. We have

$$
\begin{aligned}
\left(\partial \beta^{\text {घ }}+\beta^{\text {घh }} \partial\right)\left(x u^{2 p}\right) & =\partial\left[N(1-T)^{-1} x u^{2 p+1}\right]+\beta^{\text {घh }}\left[N x u^{2 p-1}\right] \\
& =(1-\tau) N(1-T)^{-1} x u^{2 p} .
\end{aligned}
$$

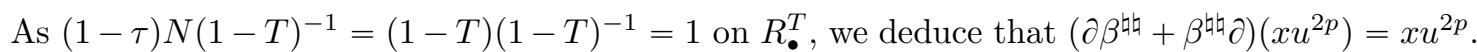

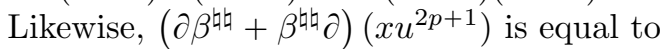

$$
\beta^{\text {叫 }}\left[(1-\tau) x u^{2 p}\right]=N(1-T)^{-1}(1-\tau) x u^{2 p+1}=x u^{2 p+1} .
$$

Therefore, we see that $\partial \beta^{\text {घต }}+\beta^{\text {घ七 }} \partial=1$ on $R_{\bullet}^{T \text {, 如 }}$.

In addition, we have

$$
\begin{aligned}
\left(\delta \beta^{\text {घ }}+\beta^{\text {घh }} \delta\right)\left(x u^{2 p}\right) & =\delta\left[N(1-T)^{-1} x u^{2 p+1}\right]+\beta^{\text {घ口 }}\left[b x u^{2 p}\right] \\
& =-b^{\prime} N(1-T)^{-1} x u^{2 p+1}+N(1-T)^{-1} b x^{2 p+1} \\
& =\left(-b^{\prime} N+N b\right)(1-T)^{-1} x u^{2 p+1} \\
& =0 .
\end{aligned}
$$

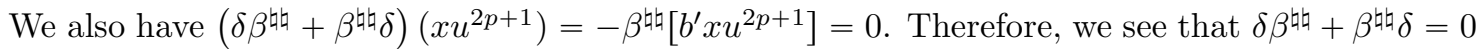
on all $R_{\bullet}^{T \text {, th }}$. The proof is complete.

The above lemma implies that $\beta^{\text {th }}$ is a contracting homotopy for $\left(R_{\bullet}^{T \text {, 蜡 }}, \partial+\delta\right)$. By construction

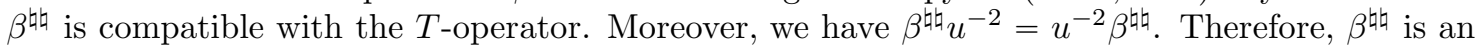

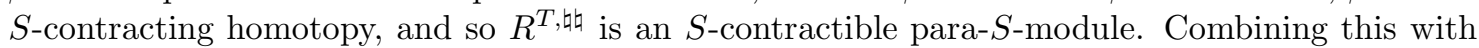
the splitting (6.4) we then arrive at the following statement.

Proposition 6.6. If $C$ is a quasi-precyclic $k$-module, then $C^{\text {घh }}$ is a quasi-S-module.

Proposition 6.6 allows us to apply Proposition 2.18 as follows. Let $\pi_{T}: C_{\bullet} \rightarrow C_{T}$ • be the canonical projection of $C_{\bullet}$ onto $C_{T, \bullet}$ and $\pi^{T}: C_{\bullet} \rightarrow C_{\bullet}$ the projection on $C_{\bullet}^{T}$ associated with the splitting $C_{\bullet}=C_{\bullet}^{T} \oplus R_{\bullet}^{T}$. They are both maps of paracyclic $k$-modules. As above, $\pi_{T}$ induces an isomorphism of para-precyclic $k$-modules from $C^{T}$ onto $C_{T}$, and, under this isomorphism, the inclusion of $C^{T}$ into $C_{\bullet}$ c corresponds to the embedding $\iota_{T}: C_{T, \bullet} \rightarrow C_{\bullet}$ given by (2.9). This embedding is actually a para-precyclic module embedding. We also let $h: C \bullet \rightarrow C_{\bullet+1}$ be the $k$-linear map defined by

$$
h^{\text {吅 }}\left(x u^{2 p}\right)=-N(1-T)^{-1}\left(x-x^{T}\right) u^{2 p+1}, \quad h^{\text {घh }}\left(x u^{2 p+1}\right)=0, \quad x \in C_{\bullet} .
$$

This is a $T$-compatible map. This is also the chain homotopy (2.10) associated with the contracting

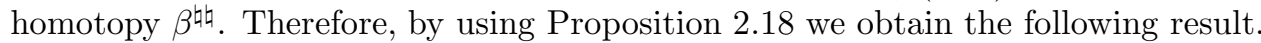

Proposition 6.7. Assume that $C$ is a quasi-precyclic $k$-module.

(1) The canonical projection $\pi_{T}: C \bullet \rightarrow C_{T} \bullet$ induces a precyclic $k$-module isomorphism from $C^{T}$ onto $C_{T}$.

(2) The map $\iota_{T}: C_{T, \bullet} \rightarrow C$ • given by (2.9) is a para-precyclic $k$-module embedding. 
(3) We have

$$
\pi_{T} \iota_{T}=1, \quad \pi^{T}=\iota_{T} \pi_{T}=1+(\partial+\delta) h^{\text {仲 }}+h^{\text {仲 }}(\partial+\delta) .
$$

This provides us with $S$-deformation retracts of $C^{\text {仲 }}$ to $C_{T}^{\text {仲 }}$ and $C^{T \text {, 如. }}$.

Remark 6.8. As we can see from (6.5), the range of $h^{\text {叫 }}$ is contained in $\oplus_{p \geqslant 0} R_{\bullet}^{T} u^{2 p+1}$ and its kernel contains $\oplus_{p \geqslant 0} C_{\bullet}^{T} u^{2 p}$ and $\oplus_{p \geqslant 0} C \cdot u^{2 p+1}$. It then follows that $\pi_{T} h^{\text {仲 }}=0, h^{\text {仲 }} \iota_{T}=0$ and $\left(h^{\text {叫 }}\right)^{2}=0$, i.e., the chain homotopy $h^{\text {叫 }}$ is special in the sense of (7.11).

Given any $r$-cyclic $k$-module $C$, Feigin-Tsygan [19, Appendix] defined a chain bicomplex, which is like the $C C$-bicomplex $\left(C C_{\bullet}, \bullet, \partial, \hat{\delta}\right)(c f$. Remark [6.3). The only difference is with the formula (6.2) for the differential $\partial$, where the operator $N$ is replaced by the operator $\sum_{j=0}^{r(m+1)-1} \tau^{j}$. As we shall now see, the Feigin-Tsygan bicomplex is a special case of a general construction for quasi-precyclic modules.

Suppose that $C$ is a quasi-precyclic $k$-module. As mentioned above the projection $\pi^{T}: C \bullet \rightarrow C$ •

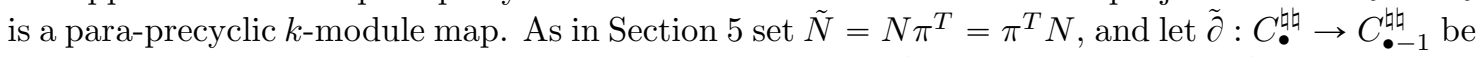
the $k$-linear map defined as in (6.2) by using the operator $\tilde{N}$ instead of $N$. Thus, $\tilde{\partial}=\pi^{T} \partial=\partial \pi^{T}$ on $C \cdot u^{2 p}$ and $\tilde{\partial}=\partial$ on $C \cdot u^{2 p+1}$. When $C$ is $r$-precyclic and $r^{-1} \in k$, the operator $\tilde{N}$ is $r^{-1}$-times the Feigin-Tsygan operator $\sum_{j=0}^{r(m+1)-1} \tau^{j}$ on $C_{m}$ (see Eq. (5.6)).

Proposition 6.9. Suppose that $C$ is a quasi-precyclic $k$-module.

(1) $\widetilde{C C}:=\left(C_{\bullet}, \bullet, \tilde{\partial}, \hat{\delta}\right)$ is a chain bicomplex and $\widetilde{C}^{\natural \natural ~}:=\left(C_{\bullet}^{\natural \natural}, \tilde{\partial}+\delta, u^{-2}\right)$ is an $S$-module.

(2) The projection $\pi^{T}: C_{\bullet} \rightarrow C_{\bullet}$ yields an $S$-homotopy equivalence between $C^{\text {印 }}$ and $\widetilde{C}^{\text {घh }}$.

Proof. We know by Lemma 6.1 that $\partial^{2}=(1-T) u^{-2}$ and $\delta^{2}=\partial \delta+\delta \partial=0$. As mentioned above $\tilde{\partial}=\pi^{T} \partial=\partial \pi^{T}$ on $C \cdot u^{2 p}$ and $\tilde{\partial}=\partial$ on $C \cdot u^{2 p+1}$. Thus, $\tilde{\partial}^{2}=\pi^{T} \delta^{2}=\pi^{T}(1-T) u^{-2}=0$. Moreover, as $\delta$ maps $C \cdot u^{q}$ to $C \cdot-1 u^{q}$ we have $\tilde{\partial} \delta+\delta \tilde{\partial}=\pi^{T}(\partial \delta+\delta \partial)=0$ on $C \bullet u^{2 p}$ and $\tilde{\partial} \delta+\delta \tilde{\partial}=\partial \delta+\delta \partial=0$ on $C \cdot u^{2 p+1}$. Therefore, we see that $\delta^{2}=\tilde{\partial}^{2}=\tilde{\partial} \delta+\delta \tilde{\partial}=0$, and hence $(\tilde{\partial}+\delta)^{2}=0$. It then follows that $\left(C C_{\bullet}, \bullet, \tilde{\partial}, \hat{\delta}\right)$ is a chain bicomplex, and so its total complex $\left(C C_{\bullet}^{\natural \natural}, \tilde{\partial}+\delta\right)$ is a chain complex. As $u^{-2}$ is compatible with the operators $(\partial, \delta)$, we then see that $\widetilde{C}^{\text {吅 }}:=\left(C C_{\bullet}^{\text {叫 }}, \tilde{\partial}+\delta, u^{-2}\right)$ is an $S$-module.

As $\left[\delta, \pi^{T}\right]=\left[u^{-2}, \pi^{T}\right]=0$ and $\tilde{\partial} \pi^{T}=\pi^{T} \tilde{\partial}=\partial \pi^{T}=\pi^{T} \partial$ we see that $\pi^{T}$ gives rise to an $S$-map

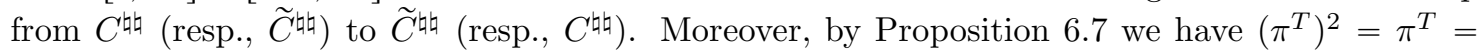

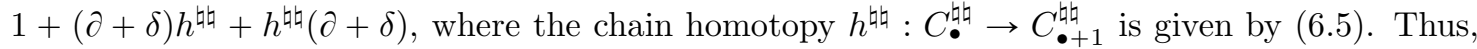

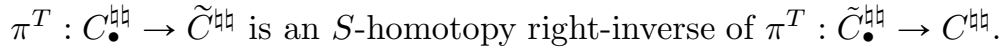

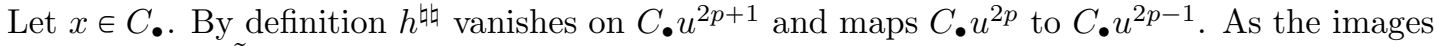
of $x u^{2 p}$ by $\partial$ and $\tilde{\partial}$ are contained in $C \cdot u^{2 p-1}$, we get

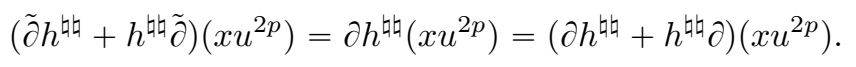

Similarly, as $h^{\text {如 }}\left(x u^{2 p+1}\right)=0$ and $\tilde{\partial}\left(x u^{2 p+1}\right)=\partial\left(x u^{2 p+1}\right)$, we have

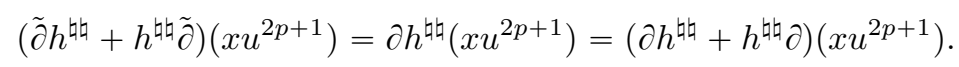

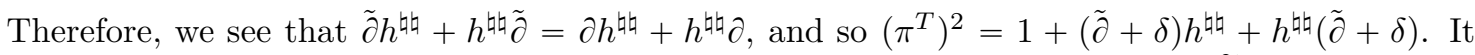

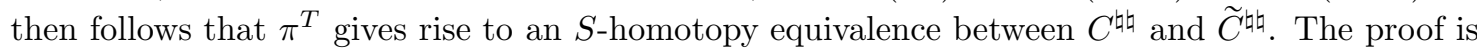
complete.

\section{Perturbation Lemmas and Co-Extensions}

The basic perturbation lemma [3, 17, 22, 53] is a simple and powerful tool for constructing chain homotopy equivalences in homological algebra (see, e.g., [1, 2, 3, 17, 22, 23, 24, 28, 34, 36, 40, 41, 52, 53]). In particular, as first observed by Kassel [34, in the context of cyclic homology it allows us to convert various deformation retracts of Hochschild complexes into deformation retracts of cyclic complexes (see also [1, 36, 151]). Incidentally, it also provides us with a natural interpretation of the $B$-operator of an $H$-unital para-precyclic module ( $c f$. [34, Remarque 5.3]; see also Section 8 ). 
In this section, we seek for generalizations of the basic perturbation lemma for para- $S$-modules, and more especially para- $S$-modules associated with parachain complexes. To this end we shall establish a generalized perturbation lemma for para-twin complexes (see below for their precise definition). Specializing these generalized perturbation lemmas to para-S-modules of parachain complexes will provide us with general recipes to convert deformation retracts of Hochschild chain complexes into $S$-deformation retracts of para- $S$-modules.

In the next sections, we will present various applications of the generalized perturbation theory of this section. We also refer to [51] for further applications regarding the normalization of the para-S-modules of paracyclic and bi-paracyclic modules and a version of the Eilenberg-Zilber theorem for bi-paracyclic modules.

7.1. Basic perturbation lemma for para-twin complexes. In what follows by a para-twin complex we shall mean a system $\left(C_{\bullet}, \partial, \delta\right)$, where $C_{m}, m \geqslant 0$, are $k$-modules and $\partial: C_{\bullet} \rightarrow C_{\bullet}-1$ and $\delta: C_{\bullet} \rightarrow C_{\bullet-1}$ are $k$-linear maps. We call $\left(C_{\bullet}, \partial, \delta\right)$ a twin complex when we further have $\partial^{2}=\delta^{2}=\partial \delta+\delta \partial=0$. In this case $\left(C_{\bullet}, \partial\right),\left(C_{\bullet}, \delta\right)$ and $\left(C_{\bullet}, \partial+\delta\right)$ are chain complexes. Given two twin complexes $\left(C_{\bullet}, \partial, \delta\right)$ and $\left(\bar{C}_{\bullet}, \partial, \delta\right)$, the basic perturbation lemma [3, 17, 22, 53 is a simple recipe for converting a deformation retract of $\left(C_{\bullet}, \partial\right)$ to $\left(\bar{C}_{\bullet}, \partial\right)$ into a deformation retract of $\left(C_{\bullet}, \partial+\delta\right)$.

We seek for generalizing the basic perturbation lemma to para-twin complexes. Thus, let $C=\left(C_{\bullet}, \partial, \delta\right)$ and $\left(\bar{C}_{\bullet}, \partial, \delta\right)$ be para-twin complexes, and assume we are given $k$-linear maps $f: C_{\bullet} \rightarrow \bar{C}_{\bullet}$ and $g: \bar{C}_{\bullet} \rightarrow C_{\bullet}$ such that

$$
g f=1+\partial \varphi+\varphi \partial,
$$

where $\varphi: C_{\bullet} \rightarrow C_{\bullet+1}$ is a $k$-linear map so that, for every $m \geqslant 0$, we have

$$
(\delta \varphi)^{j}=(\varphi \delta)^{j}=0 \quad \text { on } C_{m} \text { for } j \gg 1 .
$$

These data allow us to define $k$-linear maps $\tilde{\varphi}: C_{\bullet} \rightarrow C_{\bullet+1}, \tilde{f}: C_{\bullet} \rightarrow C_{\bullet}$ and $\tilde{g}: \bar{C} \bullet \rightarrow \bar{C}$ • by

$$
\begin{gathered}
\tilde{\varphi}=\sum_{j \geqslant 0} \varphi(\delta \varphi)^{j}=\sum_{j \geqslant 0}(\varphi \delta)^{j} \varphi, \\
\tilde{f}=f(1+\delta \tilde{\varphi}), \quad \tilde{g}=(1+\tilde{\varphi} \delta) g .
\end{gathered}
$$

Note that we have

$$
\begin{gathered}
\tilde{f}=\sum_{j \geqslant 0} f(\delta \varphi)^{j}=f+f(\delta \varphi)+f(\delta \varphi)^{2}+\cdots, \\
\tilde{g}=\sum_{j \geqslant 0}(\varphi \delta)^{j} g=g+(\varphi \delta) g+(\varphi \delta)^{2} g+\cdots,
\end{gathered}
$$

We also define the $k$-linear map $\tilde{\delta}: \bar{C}_{\bullet} \rightarrow \bar{C}_{\bullet-1}$ by

$$
\tilde{\delta}=f(\delta+\delta \tilde{\varphi} \delta) g=f \delta g+f \delta \tilde{\varphi} \delta g .
$$

We observe that

$$
\tilde{\delta}=\tilde{f} \delta g=f \delta \tilde{g}
$$

In particular, we have

$$
\tilde{\delta}=\sum_{j \geqslant 0} f(\delta \varphi)^{j} \delta g=\sum_{j \geqslant 0} f \delta(\varphi \delta)^{j} g=f \delta g+f \delta \varphi \delta g+\cdots .
$$

We also set

$$
\Delta=\delta^{2}+\partial \delta+\delta \partial
$$

We shall say that the chain homotopy $\varphi$ in (7.1) is special when the following conditions are satisfied:

$$
f \varphi=0, \quad \varphi g=0, \quad \varphi^{2}=0 .
$$

We are now in a position to obtain the following generalization of the basic perturbation lemma. 
Lemma 7.1. Suppose that the maps $f$ and $g$ are compatible with the operators $\partial$, and the chain homotopy $\varphi$ is special and $\Delta$-compatible. Then we have

$$
\begin{gathered}
\tilde{f}(\partial+\delta)=(\partial+\tilde{\delta}) \tilde{f}, \\
\tilde{g}(\partial+\tilde{\delta})=(\partial+\delta) \tilde{g}, \\
\tilde{g} \tilde{f}=1+(\partial+\delta) \tilde{\varphi}+\tilde{\varphi}(\partial+\delta), \\
\tilde{f} \tilde{g}=f g, \\
\tilde{\delta}^{2}+\partial \tilde{\delta}+\tilde{\delta} \partial=f \Delta g .
\end{gathered}
$$

In addition, the chain homotopy $\tilde{\varphi}$ is special.

Proof. We observe that (7.3) implies that

$$
\tilde{\varphi}=\varphi+\tilde{\varphi} \delta \varphi=\varphi+\varphi \delta \tilde{\varphi} .
$$

We also have the following equalities,

$$
f \Delta \varphi=0, \quad \varphi \Delta g=0, \quad \varphi \Delta \varphi=0 .
$$

Indeed, as $\varphi$ is special and compatible with $\Delta$ we have $f \Delta \varphi=f \varphi \Delta=0$. Likewise, $\varphi \Delta g=\Delta \varphi g=$ 0 and $\varphi \Delta \varphi=\Delta \varphi^{2}=0$.

By using (7.1) and (7.17) we see that $\tilde{g} \tilde{f}$ is equal to

$$
\begin{aligned}
(1+\tilde{\varphi} \delta) g f(1+\delta \tilde{\varphi})= & (1+\tilde{\varphi} \delta)(1+\partial \varphi+\varphi \partial)(1+\delta \tilde{\varphi}) \\
= & 1+\partial(\varphi+\varphi \delta \tilde{\varphi})+\delta \tilde{\varphi}+(\varphi+\tilde{\varphi} \delta \varphi) \partial+\tilde{\varphi} \delta \\
& +\tilde{\varphi} \delta \partial(\varphi+\varphi \delta \tilde{\varphi})+(\varphi+\tilde{\varphi} \delta \varphi) \partial \delta \tilde{\varphi}+\tilde{\varphi} \delta^{2} \tilde{\varphi} \\
= & 1+(\partial+\delta) \tilde{\varphi}+\tilde{\varphi}(\partial+\delta)+\tilde{\varphi} \Delta \tilde{\varphi} .
\end{aligned}
$$

We know by (7.18) that $\varphi \Delta \varphi=0$, and so $\tilde{\varphi} \Delta \tilde{\varphi}=(1+\tilde{\varphi} \delta) \varphi \Delta \varphi(1+\delta \tilde{\varphi})=0$. It then follows that $\tilde{g} \tilde{f}=1+(\partial+\delta) \tilde{\varphi}+\tilde{\varphi}(\partial+\delta)$, i.e., we have (7.14) .

By assumption $[\partial, f]=0$. Combining this with (7.8) and (7.14) gives

$$
\begin{aligned}
(\partial+\tilde{\delta}) \tilde{f} & =\partial f(1+\delta \tilde{\varphi})+f \delta \tilde{g} \tilde{f} \\
& =f \partial(1+\delta \tilde{\varphi})+f \delta[1+(\partial+\delta) \tilde{\varphi}+\tilde{\varphi}(\partial+\delta)] \\
& =(f+f \delta \tilde{\varphi}) \partial+(f+f \delta \tilde{\varphi}) \delta+f\left(\partial \delta+\delta \partial+\delta^{2}\right) \tilde{\varphi} \\
& =\tilde{f}(\partial+\delta)+f \Delta \tilde{\varphi} .
\end{aligned}
$$

We know by (7.18) that $f \Delta \varphi=0$. Thus, $f \Delta \tilde{\varphi}=f \Delta \varphi(1+\delta \tilde{\varphi})=0$, and hence $(\partial+\tilde{\delta}) \tilde{f}=\tilde{f}(\partial+\delta)$. Similarly, as by assumption $[\partial, g]=0$, we see that $\tilde{g}(\partial+\tilde{\delta})$ is equal to

$$
\begin{aligned}
(1+\tilde{\varphi} \delta) g \partial+\tilde{g} \tilde{f} \delta g & =(1+\tilde{\varphi} \delta) \partial g+(1+\partial \varphi+\varphi \partial) \delta g \\
& =\partial(g+\tilde{\varphi} \delta g)+\delta(g+\tilde{\varphi} \delta g)+\tilde{\varphi}\left(\partial \delta+\delta \partial+\delta^{2}\right) g \\
& =(\partial+\delta) \tilde{g}+\tilde{\varphi} \Delta g .
\end{aligned}
$$

As by (7.18) we have $\varphi \Delta g=0$, we get $\tilde{\varphi} \Delta g=(1+\tilde{\varphi} \delta) \varphi \Delta g=0$, and so $\tilde{g}(\partial+\tilde{\delta})=(\partial+\delta) \tilde{g}$.

By using (7.8) and (7.14) we see that $\tilde{\delta}^{2}$ is equal to

$$
\begin{aligned}
f \delta \tilde{g} \tilde{f} \delta g & =f \delta[1+(\partial+\delta) \tilde{\varphi}+\tilde{\varphi}(\partial+\delta)] \delta g \\
& =f \delta^{2}+f\left(\delta^{2}+\delta \partial\right) \tilde{\varphi} \delta g+f \delta \tilde{\varphi}\left(\delta^{2}+\partial \delta\right) g \\
& =f \Delta g+f \Delta \tilde{\varphi} \delta g+f \delta \tilde{\varphi} \Delta g-f(\partial \delta+\delta \partial) g-f \partial \delta \tilde{\varphi} \delta g-f \delta \tilde{\varphi} \delta \partial g .
\end{aligned}
$$

By using the compatibility of $f$ and $g$ with the $\partial$-operators and the equalities $f \Delta \tilde{\varphi}=0$ and $\tilde{\varphi} \Delta g=0$ we obtain

$$
\begin{aligned}
\tilde{\delta}^{2} & =f \Delta g-\partial f \delta-\delta g \partial-\partial f \delta \tilde{\varphi} \delta g-f \delta \tilde{\varphi} \delta g \partial \\
& =f \Delta g-\partial f(\delta+\delta \tilde{\varphi} \delta) g-f(\delta+\delta \tilde{\varphi} \delta) g \\
& =f \Delta g-\partial \tilde{\delta}-\tilde{\delta} \partial .
\end{aligned}
$$


This proves (7.16).

It remains to show that $\tilde{f} \tilde{g}=f g$ and $\tilde{\varphi}$ is special. We have $\tilde{\varphi}^{2}=(1+\tilde{\varphi} \delta) \varphi^{2}(1+\delta \tilde{\varphi})=0$. Combining this with (7.4) we get

$$
\tilde{f} \tilde{g}=f(1+\delta \tilde{\varphi})(1+\tilde{\varphi} \delta) g=f g+f \tilde{\varphi} \delta g+f \delta \tilde{\varphi} g .
$$

We also have $f \tilde{\varphi}=f \varphi(1+\delta \tilde{\varphi})=0$ and $\tilde{\varphi} g=(1+\tilde{\varphi} \delta) \varphi g=0$. Thus,

$$
\begin{gathered}
\tilde{f} \tilde{\varphi}=f(1+\delta \tilde{\varphi}) \tilde{\varphi}=f \tilde{\varphi}+f \delta \tilde{\varphi}^{2}=0, \\
\tilde{\varphi} \tilde{g}=\tilde{\varphi}(1+\tilde{\varphi} \delta) g=\tilde{\varphi} g+\tilde{\varphi}^{2} \delta g=0 .
\end{gathered}
$$

Combining this with (7.19) shows that $\tilde{f} \tilde{g}=f g . \quad$ As $\tilde{\varphi}^{2}=0$ this also shows that the chain homotopy $\tilde{\varphi}$ is special. The proof is complete.

Remark 7.2. When $f g=1$ and $C$ and $\bar{C}$ are both twin complexes (i.e., $\partial^{2}=\delta^{2}=\partial \delta+\delta \partial=0$ ) we recover the basic perturbation lemma in the version of $[3,22$.

Remark 7.3. When $f g=1$ and $[\Delta, f]=0$ the equalities (7.15) and (7.16) become

$$
\tilde{f} \tilde{g}=f g=1 \quad \text { and } \quad \tilde{\delta}^{2}+\partial \tilde{\delta}+\tilde{\delta} \partial=f \Delta g=\Delta f g=\Delta .
$$

Remark 7.4. The proof of Lemma 7.1] shows a little more. A close examination of the proof shows the following:

(a) In order to get (7.14) we only need (7.18)

(b) In order to get (17.12) (resp., (77.13)) we only need (7.18) and the compatibility of the map $f$ (resp., $g$ ) with the $\partial$-operators.

(c) In order to get (7.16) we only need (7.18) and the compatibility with the $\partial$-operators of both maps $f$ and $g$.

Remark 7.5. If $[\delta, f]=0$, then $\tilde{f}=f$ and $\tilde{\delta}=f \delta g=\delta f g$. Indeed, as $\varphi$ is special, we have $f \delta \varphi=\delta f \varphi=0$, and so by using (7.5) and (7.9) we see that $\tilde{f}=f$ and $\tilde{\delta}=f \delta g$. In particular, when $[\delta, f]=0$ and $f g=1$ we have $\tilde{\delta}=\delta$.

Remark 7.6. The assumption that $\varphi$ is special ensures us that $f \varphi=0$ and $\varphi g=0$. When $f g=1$ these two conditions are not essential. Indeed, if we set $\pi=g f$, then $\pi$ is compatible with the $\partial$ operator on $C$ • and, as $f g=1$, we have $\pi^{2}=g(f g) f=\pi$. Therefore, if we set $\tilde{\varphi}=(1-\pi) \varphi(1-\pi)$, then we have

$$
f g-1=(1-\pi)(f g-1)(1-\pi)=(1-\pi)(\partial \varphi+\varphi \partial)(1-\pi)=\partial \tilde{\varphi}+\tilde{\varphi} \partial .
$$

We also have $f(1-\pi)=f-(f g) f=0$ and $(1-\pi) g=g-g(f g)=0$, and so $f \tilde{\varphi}=0$ and $\tilde{\varphi} g=0$. Thus, upon replacing $\varphi$ by $\tilde{\varphi}$ we obtain a chain homotopy such that $f \varphi=0$ and $\varphi g=0$.

Assume further that $\partial^{2}=0$ and we are given a chain homotopy such that $f \varphi=0$ and $\varphi g=0$. Under these assumptions the condition $\varphi^{2}=0$ in (7.11) becomes inessential. Indeed, we then have $(\partial \varphi)^{2}=(1-\pi-\varphi \partial) \partial \varphi=\partial \varphi$. Likewise, $(\varphi \partial)^{2}=\varphi \partial$, and so $\partial(\varphi \partial \varphi)+(\varphi \partial \varphi) \partial=\partial \varphi+\varphi \partial=1-\pi$. We also have $\partial \varphi^{2} \partial=(1-\pi-\varphi \partial)(1-\pi-\partial \varphi)=1-\pi-\varphi \partial-\partial \varphi=0$, and hence $(\varphi \partial \varphi)^{2}=\varphi\left(\partial \varphi^{2} \partial\right) \varphi=0$. Thus, upon replacing $\varphi$ by $\varphi \partial \varphi$ we obtain a chain homotopy such that $\varphi^{2}=0$.

All this shows that, when $f g=1$ and $\partial^{2}=0$, we can convert $\varphi$ into a special homotopy by replacing it by

$$
\hat{\varphi}:=\tilde{\varphi} \partial \tilde{\varphi}=(1-\pi) \varphi \partial(1-\pi) \varphi(1-\pi) .
$$

Note that when $f \varphi=0$ we simply have $\hat{\varphi}=\varphi \partial \varphi(1-\pi)$.

When $f g \neq 1$ or $\partial^{2}=0$ the above transformations need apply. Thus, whereas in the setting of the basic perturbation lemma the special homotopy assumption is not essential, it becomes essential for the generalization provided by Lemma 7.1 .

Further elaborating on the remarks above we would like to stress that, when $\Delta=0$ and $f$ is compatible with the $\delta$-operators, we actually can relax the assumption on $\varphi$ being special (compare [34]). Namely, we have the following result. 
Lemma 7.7. Suppose that $\Delta=0$ on $C_{\bullet}$ and $\bar{C}_{\bullet}$. Assume further that $[\delta, f]=f \varphi=0$ and $[\partial, g]=0$. Then we have

$$
\begin{gathered}
(\partial+\delta) \tilde{g}=\tilde{g}(\partial+\tilde{\delta}) \\
f \tilde{g}=f g, \quad \tilde{g} f=1+(\partial+\delta) \tilde{\varphi}+(\partial+\delta) \tilde{\varphi} \\
\tilde{\delta}=\delta f g, \quad \tilde{\delta}^{2}+\partial \tilde{\delta}+\tilde{\delta} \partial=0 \\
f \tilde{\varphi}=0 .
\end{gathered}
$$

Proof. As $[\delta, f]=f \varphi=0$, we know by Remark 7.5 that $\tilde{f}=f$ and $\tilde{\delta}=\delta f g$. Moreover, in the same way as in the proof of Lemma 7.1, we have $f \tilde{\varphi}=f \varphi(1+\delta \tilde{\varphi})=0$, and so $f \delta \tilde{\varphi}=\delta f \tilde{\varphi}=0$. Combining this with (7.4) we get $f \tilde{g}=\tilde{f} \tilde{g}=f(1+\delta \tilde{\varphi})(1+\tilde{\varphi} \delta) g=f g$.

By assumption $[\partial, g]=0$, and as $\Delta=0$ the equalities (7.18) are trivially satisfied. Therefore, by Remark 7.4 we have (7.13), (7.14) and (7.16). As $\tilde{f}=f$ and $\Delta=0$ this gives (7.21) and the equalities $\tilde{g} f=\tilde{g} \tilde{f}=1+(\partial+\delta) \tilde{\varphi}+(\partial+\delta) \tilde{\varphi}$ and $\tilde{\delta}^{2}+\partial \tilde{\delta}+\tilde{\delta} \partial=f \Delta g=0$. The proof is complete.

Remark 7.8. When $f g=1$ we have $f \tilde{g}=f g=1$ and $\tilde{\delta}=\delta f g=\delta$, and so $(\partial+\delta) \tilde{g}=\tilde{g}(\partial+\delta)$. Thus, if we further have the compatibility of $f$ with the $\partial$-operators, then we obtain a deformation retract of $\left(C_{\bullet}, \partial+\delta\right)$ to $\left(\bar{C}_{\bullet}, \partial+\delta\right)$.

7.2. Perturbation lemmas for parachain complexes. We shall now specialize Lemma 7.1 and Lemma 7.7 to parachain complexes. This will enable us to construct co-extensions of deformation retracts of Hochschild complexes. In particular, this will generalize to parachain complexes the version of the basic perturbation lemma for mixed complexes of Kassel 34 (see also 11).

Suppose that $C=\left(C_{\bullet}, b, B\right)$ and $\bar{C}=\left(\bar{C}_{\bullet}, b, B\right)$ are parachain complexes. Any $S$-map $f: C_{\bullet}^{\natural} \rightarrow$ $\bar{C}_{\bullet}^{\natural}$ is of the form $f=f^{(0)} u^{0}+f^{(1)} u+\cdots$, where $f^{(j)}: C_{\bullet} \rightarrow \bar{C}_{\bullet}+2 j$ are $T$-compatible $k$-linear maps satisfying (3.3). In particular, the zero-th degree component $f^{(0)}: C_{\bullet} \rightarrow \bar{C} \bullet$ is a Hochschild chain map, i.e., $\left[b, f^{(0)}\right]=0$. Conversely, given any $T$-compatible Hochschild chain map $f: C_{\bullet} \rightarrow \bar{C}_{\bullet}$, we shall call co-extension of $f$ any $S$-map $f^{\natural}: C_{\bullet}^{\natural} \rightarrow \bar{C}_{\bullet}^{\natural}$ such that $f^{\natural(0)}=f$. This terminology was coined by Hood-Jones 27 .

Throughout the rest of this section we assume we are given Hochschild chain maps $f: C \bullet \rightarrow \bar{C}$ • and $g: \bar{C}_{\bullet} \rightarrow C_{\bullet}$ that provide us with a deformation retract,

$$
f g=1, \quad g f=1+b \varphi+\varphi b,
$$

where $\varphi: C_{\bullet} \rightarrow C_{\bullet+1}$ is some $k$-linear map. We seek for applying Lemma 7.1 to the the para-twin complexes $\left(C_{\bullet}^{\natural}, b, B u^{-1}\right)$ and $\left(\bar{C}_{\bullet}^{\natural}, b, B u^{-1}\right)$. Note that the nilpotency of $\delta=B u^{-1}$ ensures us that the condition (7.2) is automatically satisfied.

Let $\varphi^{\natural}: C_{\bullet}^{\natural} \rightarrow C_{\bullet+1}^{\natural}$ be the $k$-linear map defined by

$$
\varphi^{\natural}=\sum_{j \geqslant 0} \varphi(B \varphi)^{j} u^{-j}=\varphi+\varphi B \varphi u^{-1}+\cdots .
$$

We also define $k$-linear maps $f^{\natural}: C_{\bullet}^{\natural} \rightarrow \bar{C}_{\bullet}^{\natural}$ and $g^{\natural}: \bar{C}_{\bullet}^{\natural} \rightarrow C_{\bullet}^{\natural}$ by

$$
\begin{gathered}
f^{\natural}=f+f B \varphi^{\natural} u^{-1}=f+\sum_{j \geqslant 1} f(B \varphi)^{j} u^{-j}, \\
g^{\natural}=g+\varphi^{\natural} B g u^{-1}=g+\sum_{j \geqslant 1}(\varphi B)^{j} g u^{-j} .
\end{gathered}
$$

Note that the maps $f^{\natural}$ and $g^{\natural}$ are compatible with the $S$-operators and their zero-th degree componenents are $f$ and $g$, respectively. In addition, we let $\tilde{B}: \bar{C}_{\bullet} \rightarrow \bar{C}_{\bullet+1}$ be the $k$-linear map defined by

$$
\tilde{B}=\sum_{j \geqslant 0} \varphi f(B \varphi)^{j} B g u^{-j}=f B g+f B \varphi B g+\cdots .
$$

By assumption the chain homotopy $\varphi$ is special. Note also that if $\partial=b$ and $\delta=B u^{-1}$, then in the notation of (7.3) -(7.7) the maps $\left(\varphi^{\natural}, f^{\natural}, g^{\natural}, \tilde{B} u^{-1}\right)$ are precisely the maps $(\tilde{\varphi}, \tilde{f}, \tilde{g}, \tilde{\delta})$. Moreover, 
we have

$$
\Delta=\delta^{2}+\partial \delta+\delta \partial=B^{2} u^{-2}+(b B+B b) u^{-1}=(1-T) u^{-1} .
$$

Thus, the compatibility with the $\Delta$-operators follows from the compatibility with the $T$-operators. Therefore, we have the following version of Lemma 7.1 .

Lemma 7.9. Suppose that the maps $(f, g, \varphi)$ are T-compatible and the chain homotopy $\varphi$ is special. Then

(i) $\tilde{C}^{\natural}:=\left(\bar{C}_{\bullet}^{\natural}, b+\tilde{B} u^{-1}, u^{-1}, T\right)$ is a para-S-module.

(ii) $f^{\natural}: C_{\bullet}^{\natural} \rightarrow \tilde{C}_{\bullet}^{\natural}$ and $g^{\natural}: \tilde{C}_{\bullet}^{\natural} \rightarrow \tilde{C}_{\bullet}^{\natural}$ are $S$-maps such that

$$
f^{\natural} g^{\natural}=1, \quad g^{\natural} f^{\natural}=1+\left(b+B u^{-1}\right) \varphi^{\natural}+\varphi^{\natural}\left(b+B u^{-1}\right) .
$$

In particular, we obtain an $S$-deformation retract of $C^{\natural}$ to $\tilde{C}^{\natural}$, and a $T$-deformation retract of $C^{\sharp}$ to $\tilde{C}^{\sharp}$.

(iii) The chain homotopy $\varphi^{\natural}$ is special.

Proof. The $T$-compatibility of the maps $(f, g, \varphi)$ ensure us the $T$-compatibility of the maps $\left(f^{\natural}, g^{\natural}, \varphi^{\natural}\right)$. Granted this, the parts (ii) and (iii) then follow from the last two parts of Lemma 7.1 and from Remark 7.3. Moreover, by using (7.20) and (7.26) and the fact that $b^{2}=0$ we get

$$
(1-T) u^{-1}=\tilde{B}^{2} u^{-2}+(b \tilde{B}+\tilde{B} b) u^{-1}=\left(b+\tilde{B} u^{-1}\right)^{2} .
$$

As $\tilde{B}$ is compatible with $u^{-1}$ and $T$ it then follows that $\left(\bar{C}_{\bullet}^{\natural}, b+\tilde{B} u^{-1}, u^{-1}, T\right)$ is a para- $S$-module. The proof is complete.

Remark 7.10. When $C$ and $\bar{C}$ are mixed complexes (i.e., $b B+B b=0$ ) the para- $S$-module $\tilde{C}^{\natural}$ is actually an $S$-module, since in this case $T=1-(b B+B b)=1$. In particular, we recover the version of the basic perturbation lemma for mixed complexes of Kassel [34] (see also [1, Lemma I.2]).

Remark 7.11. Write $B=\sum_{j \geqslant 0} B^{(j)} u^{-j}$, with $B^{(j)}:=f(B \varphi)^{j} B g$. Then (7.27) implies that

$$
b B^{(0)}+B^{(0)} b=1-T, \quad \sum_{p+q=j-1} B^{(p)} B^{(q)}+b B^{(j)}+B^{(j)} b=0, \quad j \geqslant 1 .
$$

Thus, if $B^{(j)}=0$ for $j \geqslant 1$, then $\left(\bar{C}, b, B^{(0)}\right)$ is a parachain complex whose para- $S$-module is precisely $\tilde{C}^{\natural}$.

Remark 7.12. In general, $\tilde{C}^{\natural}$ need not be the para-S-module of a parachain complex. This shows the relevance of considering para- $S$-modules when attempting to apply perturbation lemmas in the setting of parachain complexes.

Further elaborating on Remark 7.11 we have the following result.

Lemma 7.13. Suppose that the maps $(f, g, \varphi)$ are $T$-compatible and the chain homotopy $\varphi$ is special. Assume further that

$$
f B g=B \quad \text { and } \quad f(B \varphi)^{j} B g=0 \quad \text { for } j \geqslant 1 .
$$

Then the following holds.

(i) $f^{\natural}: C_{\bullet}^{\natural} \rightarrow \bar{C}_{\bullet}^{\natural}$ and $g^{\natural}: \bar{C}_{\bullet}^{\natural} \rightarrow C_{\bullet}^{\natural}$ are $S$-maps and coextensions of $f$ and $g$, respectively.

(ii) We have

$$
f^{\natural} g^{\natural}=1, \quad g^{\natural} f^{\natural}=1+\left(b+B u^{-1}\right) \varphi^{\natural}+\varphi^{\natural}\left(b+B u^{-1}\right) .
$$

In particular, we obtain an $S$-deformation retract of $C^{\natural}$ to $\bar{C}^{\natural}$ and a $T$-deformation retract of $C^{\sharp}$ to $\bar{C}^{\sharp}$.

(iii) The chain homotopy $\varphi^{\natural}$ is special.

Proof. The assumptions (7.28) and Remark 7.11 ensure us that the para-S-module $\tilde{C}^{\natural}$ of the first part of Lemma 7.9 agrees with $\bar{C}^{\natural}$. The result then follows from the last two parts of Lemma 7.9 .

When $f$ is already a map of parachain complexes we have the following statement. 
Lemma 7.14. Suppose that $f$ is a map of parachain complexes, the maps $(g, \varphi)$ are $T$-compatible, and the chain homotopy $\varphi$ is special. Then

(i) $g^{\natural}: \bar{C}_{\bullet}^{\natural} \rightarrow C_{\bullet}^{\natural}$ is an $S$-map and a co-extension of $g$.

(ii) We have

$$
f g^{\natural}=1, \quad g^{\natural} f=1+\left(b+B u^{-1}\right) \varphi^{\natural}+\varphi^{\natural}\left(b+B u^{-1}\right) .
$$

In particular, this provides us with an $S$-deformation retract of $C^{\natural}$ to $\bar{C}^{\natural}$ and a $T$-deformation retract of $C^{\sharp}$ to $\bar{C}^{\sharp}$.

(iii) The chain homotopy $\varphi^{\natural}$ is special.

Proof. The fact that $f$ is a map of parachain complexes ensures us it is compatible with the operators $T=1-(b B+B b)$ and $\delta=B u^{-1}$. As $f g=1$, we can argue in the same way as in Remark 7.5 to show that $f^{\natural}=f$ and the conditions (7.28) are satisfied. The result then follows from Lemma 7.13 .

Finally, when $C$ and $\bar{C}$ are mixed complexes (i.e., when $T=1$ ) we can relax the assumptions on the chain homotopy $\varphi$ (compare [1, 34). Namely, by using Lemma 7.7 and Remark 7.8 we obtain the following result.

Lemma 7.15. Suppose that $C$ and $\bar{C}$ are mixed complexes. Assume further that $f$ is a map of mixed complexes and $f \varphi=0$. Then

(i) $g^{\natural}: \bar{C}_{\bullet}^{\natural} \rightarrow C_{\bullet}^{\natural}$ is an $S$-map and a co-extension of $g$.

(ii) We have

$$
f g^{\natural}=1, \quad g^{\natural} f=1+\left(b+B u^{-1}\right) \varphi^{\natural}+\varphi^{\natural}\left(b+B u^{-1}\right), \quad f \varphi^{\natural}=0 .
$$

This provides us with an $S$-deformation retract of $C^{\natural}$ to $\bar{C}^{\natural}$ and a $T$-deformation retract of $C^{\sharp}$ to $\bar{C}^{\sharp}$.

7.3. Some Applications. It is well known that a map of mixed complexes is a quasi-isomorphism at the cyclic chain level if and only if it is an isomorphism at the ordinary chain level (see, e.g., [42]). As a first application of the results of this section we shall prove the following version of that result for parachain complexes.

Proposition 7.16. Suppose that $C=(C, b, B)$ and $\bar{C}=\left(\bar{C}_{\bullet}, b, B\right)$ are parachain complexes, and let $f: C \bullet \rightarrow \bar{C}$. be a parachain complex map. Then the following are equivalent:

(i) $f$ gives rise to a $T$-deformation retract of $\left(C_{\bullet}, b\right)$ to $\left(\bar{C}_{\bullet}, b\right)$.

(ii) $f$ gives rise to an $S$-deformation retract of $C^{\natural}$ to $\bar{C}^{\natural}$.

Furthermore, if (i) and (ii) hold, then we get a T-deformation retract of $C^{\sharp}$ to $\bar{C}^{\sharp}$.

Proof. Suppose that $f$ gives rise to a $T$-deformation retract of $\left(C_{\bullet}, b\right)$ to $\left(\bar{C}_{\bullet}, b\right)$. That is, there is a $T$-compatible chain map $g:\left(\bar{C}_{\bullet}, b\right) \rightarrow\left(C_{\bullet}, b\right)$ and a $T$-compatible $k$-linear map $\varphi: C_{\bullet} \rightarrow C_{\bullet}+1$ satisfying (7.25). As pointed out in Remark [7.6, we may assume that the chain homotopy $\varphi$ is special, since $f g=1$ and $b^{2}=0$. Lemma 7.14 then produces a coextension $g^{\natural}: \bar{C}_{\bullet}^{\natural} \rightarrow C_{\bullet}^{\natural}$ which is a right-inverse of $f$ on $\bar{C}^{\natural}$ and a $S$-homotopy left-inverse on $C_{\bullet}^{\natural}$. This gives an $S$-deformation retract of $C^{\natural}$ to $\bar{C}^{\natural}$, and so by Proposition 2.9 we obtain a $T$-deformation retract of $C^{\sharp}$ to $\bar{C}^{\sharp}$.

Conversely, suppose there are an $S$-map $g^{\natural}: \bar{C}_{\bullet}^{\natural} \rightarrow C_{\bullet}^{\natural}$ and an $(S, T)$-compatible $k$-linear map $\varphi^{\natural}: C_{\bullet}^{\natural} \rightarrow C_{\bullet+1}^{\natural}$ such that

$$
f g^{\natural}=1, \quad g^{\natural} f=1+\left(b+B u^{-1}\right) \varphi^{\natural}+\varphi^{\natural}\left(b+B u^{-1}\right) .
$$

Set $g^{\natural}=\sum_{j \geqslant 0} g^{(j)} u^{-j}$ and $\varphi^{\natural}=\sum_{j \geqslant 0} \varphi^{(j)} u^{-j}$, where $g^{(j)}: C_{\bullet} \rightarrow C_{\bullet+2 j}$ and $\varphi^{(j)}: C_{\bullet} \rightarrow C_{\bullet}+2 j+1$ are $T$-compatible $k$-linear maps. We observe that the zeroth degree component parts of $f g^{\natural}$, $g^{\natural} f$, and $1+\left(b+B u^{-1}\right) \varphi^{\natural}+\varphi^{\natural}\left(b+B u^{-1}\right)$ are $f g^{(0)}, g^{(0)} f$, and $1+b \varphi^{(0)}+\varphi^{(0)} b$, respectively. Therefore, taking zeroth degree component parts in both equalities in (7.29) gives $f g^{(0)}=1$ and $g^{(0)} f=1+b \varphi^{(0)}+\varphi^{(0)} b$. In particular, we get a $T$-deformation retract of $\left(C_{\bullet}, b\right)$ to $\left(\bar{C}_{\bullet}, b\right)$. The proof is complete. 
Applying the above result to the canonical projection $\pi_{T}: C_{\bullet} \rightarrow C_{T, \bullet}$ of a parachain complex provides us with the following characterization of property (DR) for para- $S$-modules associated with parachain complexes.

Corollary 7.17. Let $C=\left(C_{\bullet}, b, B\right)$ be a parachain complex. Then the following are equivalent:

(i) The para-S-module $C^{\natural}$ has property (DR).

(ii) The canonical projection $\pi_{T}: C_{\bullet} \rightarrow C_{T, \bullet}$ gives rise to a $T$-deformation retract of $\left(C_{\bullet}, b\right)$ to $\left(C_{T, \bullet}, b\right)$.

Remark 7.18. A parachain complex $\left(C_{\bullet}, b, B\right)$ such that the canonical projection $\pi_{T}:\left(C_{\bullet}, b\right) \rightarrow$ $\left(C_{T, \bullet}, b\right)$ is a quasi-isomorphism is called a homological skycraper in [38.

\section{Comparison of the Para-S-Modules $C^{\text {घh }}$ and $C^{\natural}$}

When $C$ is a cyclic module, Connes 8 showed that $C^{\text {घ }}$ and $C^{\natural}$ are quasi-isomorphic chain complexes (see also [42, 43]). Subsequently, Kassel 34 exhibited a deformation retract of $C^{\text {如 }}$ to $C^{\natural}$ when $C$ is an $H$-unital precyclic module. In addition, he pointed out that his deformation retract could be obtained by using the basic perturbation lemma, and the $B$-operator would naturally re-appear from this process ( $c f$. [34, Remarque 5.3]).

In this section, by elaborating on Kassel's observation we shall extend to $H$-unital para-precyclic modules the aforementioned equivalence results. This will use the generalization of the basic perturbation lemma given by Lemma 7.1. In particular, as we shall see, and further confirming Kassel's observation, the $B$-differential (5.5) naturally arises from the perturbation of the deformation retract of $\left(C_{\bullet}^{\natural \natural}, \delta\right)$ to $\left(C_{\bullet}^{\natural}, b\right)$.

The deformation retract of $\left(C_{\bullet}^{\natural \natural}, \delta\right)$ to $\left(C_{\bullet}^{\natural}, b\right)$ is obtained as follows. We have a natural $k$-module embedding $I_{0}: C_{\bullet}^{\natural} \rightarrow C_{\bullet}^{\natural \natural}$ given by

$$
I_{0}\left(x u^{p}\right)=x u^{2 p}, \quad x \in C_{\bullet} .
$$

As $I_{0} b\left(x u^{p}\right)=I_{0}\left(b x u^{p}\right)=b x u^{2 p}=\delta\left(x u^{2 p}\right)=\delta I_{0}\left(x u^{p}\right)$, we see that $I_{0}$ is a chain map from $\left(C_{\bullet}^{\natural}, b\right)$

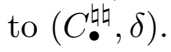

We also have a natural projection $J_{0}: C_{\bullet}^{\natural \natural} \rightarrow C_{\bullet}^{\natural}$ given by

$$
J_{0}\left(x u^{2} p\right)=x u^{p}, \quad J_{0}\left(x u^{2 p+1}\right)=0 .
$$

As $J_{0} \delta\left(x u^{2 p}\right)=J_{0}\left(b x u^{2 p}\right)=b x u^{p}=b J_{0}\left(x u^{2 p}\right)$ and $J_{0} \delta\left(x u^{2 p+1}\right)=J_{0}\left(b x u^{2 p+1}\right)=0=b J_{0}\left(x u^{2 p+1}\right)$, we also see that $J_{0}$ is a chain map from $\left(C_{\bullet}^{\text {घ乌 }}, \delta\right)$ to $\left(C_{\bullet}^{\natural}, b\right)$.

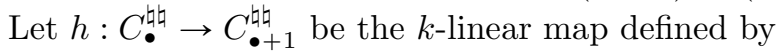

$$
h\left(x u^{2 p}\right)=0, \quad h\left(x u^{2 p+1}\right)=s^{\prime} x u^{2 p+1} .
$$

Lemma 8.1. We have

$$
J_{0} I_{0}=1, \quad I_{0} J_{0}=1+\delta h+h \delta .
$$

In particular, we get a deformation retract of $\left(C C_{\bullet}^{\natural \natural}, \delta\right)$ to $\left(C_{\bullet}^{\natural}, b\right)$. In addition, the chain homotopy $h$ is special.

Proof. Let $x \in C_{\text {. }}$. We have $J_{0} I_{0}\left(x u^{p}\right)=J_{0}\left(x u^{p}\right)=1$, and so $J_{0} I_{0}=1$. Likewise, we have $I_{0} J_{0}\left(x u^{2 p}\right)=I_{0}\left(x u^{p}\right)=x u^{2 p}$. As $h=0$ on $C \cdot u^{2 p}$, we also get $(\delta h+h \delta)\left(x u^{2 p}\right)=h\left[b x u^{2 p}\right]=0$. Thus,

$$
(1+\delta h+h \delta)\left(x u^{2 p}\right)=x u^{2 p}=I_{0} J_{0}\left(x u^{2 p}\right) .
$$

Note also that $I_{0} J_{0}\left(x u^{2 p+1}\right)=0$. Moreover, we have

$$
(\delta h+h \delta)\left(x u^{2 p+1}\right)=\delta\left[s^{\prime} x u^{2 p+1}\right]-h\left[b^{\prime} x u^{2 p+1}\right]=-\left(b^{\prime} s^{\prime}+s^{\prime} b^{\prime}\right) x u^{2 p+1}=-x u^{2 p+1} .
$$

Therefore, we see that $(1+\delta h+h \delta)\left(x u^{2 p+1}\right)=0=I_{0} J_{0}\left(x u^{2 p+1}\right)$. Combining this with (8.2) gives the homotopy formula $I_{0} J_{0}=1+\delta h+h \delta$.

In addition, we have $h^{2}\left(x u^{2 p}\right)=0$ and $h^{2}\left(x u^{2 p+1}\right)=\left(s^{\prime}\right)^{2} x u^{2 p+1}=0$, and so $h^{2}=0$. As $h I_{0}\left(x u^{p}\right)=h\left(x u^{2 p}\right)=0$, we also see that $h I_{0}=0$. In addition, we have $J_{0} h\left(x u^{2 p}\right)=0$ and 
$J_{0} h\left(x u^{2 p+1}\right)=J_{0}\left(s^{\prime} x u^{2 p+1}\right)=0$, and so $J_{0} h=0$. All this shows that the chain homotopy $h$ is special. The proof is complete.

We seek for applying Lemma 7.1 to the deformation retract (8.1). That is, for the paratwin complexes $\left(C^{\natural \natural}, \delta, \partial\right)$ and $\left(C_{\bullet}^{\natural}, b, B u^{-1}\right)$, where the respective roles of the maps $(f, g, \varphi)$ are played by the maps $\left(J_{0}, I_{0}, h\right)$. As $\left(C_{\bullet}^{\natural}, b, B u^{-1}\right)$ is a parachain complex, its $\Delta$-operator (17.10) is $(1-T) u^{-1}$. Using Lemma 6.1 we also see that the $\Delta$-operator of $\left(C C^{\natural \natural}, \delta, \partial\right)$ is given by

$$
\Delta=\partial^{2}+\delta \partial+\partial \delta=(1-T) u^{-2} .
$$

It is immediate from their definitions that the maps $\left(J_{0}, I_{0}, h\right)$ are $T$-compatible. It is also straightforward to check that $J_{0} u^{-2}=u^{-1} J_{0}, I_{0} u^{-1}=u^{-2} I_{0}$ and $h u^{-2}=u^{-2} h$. Therefore, we see that the maps $\left(J_{0}, I_{0}, h\right)$ are $\Delta$-compatible. Together with Lemma 8.1 this allows us to apply Lemma 7.1 .

Let $(\tilde{h}, \tilde{J}, \tilde{I}, \tilde{\partial})$ be the maps (7.3) $-(7.4)$ and (7.7) associated with $\left(h, J_{0}, I_{0}, \partial\right)$. Namely,

$$
\tilde{h}=\sum_{j \geqslant 0} h(\partial h)^{j}, \quad \tilde{J}=J_{0}(1+\partial \tilde{h}), \quad \tilde{I}=(1+\tilde{h} \partial) I_{0}, \quad \tilde{\partial}=J_{0}(\partial+\partial \tilde{h} \partial) I_{0} .
$$

By Lemma 7.1 these maps provide us with a deformation retract from $\left(C_{\bullet}^{\natural \natural}, \delta+\partial\right)$ to $\left(C_{\bullet}^{\natural}, b+\tilde{\partial}\right)$. It just remains to identify them.

Let $I: C_{\bullet}^{\natural} \rightarrow C_{\bullet}^{\natural \natural}$ be the $k$-linear map defined by

$$
I\left(x u^{0}\right)=x u^{0}, \quad I\left(x u^{p}\right)=x u^{2 p}+s^{\prime} N x u^{2 p-1}, \quad p \geqslant 1 .
$$

We also define the $k$-linear map $J: C_{\bullet}^{\natural \natural ~} \rightarrow C_{\bullet}^{\natural}$ by

$$
J\left(x u^{2 p}\right)=x u^{p}, \quad J\left(x u^{2 p+1}\right)=(1-\tau) s^{\prime} x u^{p}, \quad p \geqslant 0 .
$$

Lemma 8.2. We have

$$
\tilde{h}=h, \quad \tilde{J}=J, \quad \tilde{I}=I, \quad \tilde{\partial}=B u^{-1} .
$$

Proof. Let $x \in C_{\text {. . We have }}$

$$
\partial h\left(x u^{2 p}\right)=0 \quad \text { and } \quad \partial h\left(x u^{2 p+1}\right)=\partial\left(s^{\prime} x u^{2 p+1}\right)=(1-\tau) s^{\prime} x u^{2 p} .
$$

This implies that $h \partial h\left(x u^{2 p}\right)=0$ and $h \partial h\left(x u^{2 p+1}\right)=h\left[(1-\tau) s^{\prime} x u^{2 p}\right]=0$, and hence $h \partial h=0$. It then follows that $\tilde{h}=\sum_{j \geqslant 0} h(\partial h)^{j}=h$. As a result we can substitute $h$ for $\tilde{h}$ in the definitions of $(\tilde{I}, \tilde{J}, \tilde{\partial})$ in 8.3 .

By using (8.6) we get $\tilde{J}\left(x u^{2 p}\right)=J_{0}(1+\partial h)\left(x u^{2 p}\right)=J_{0}\left(x u^{2 p}\right)=x u^{p}$. We also get

$$
\tilde{J}\left(x u^{2 p}\right)=J_{0}(1+\partial h)\left(x u^{2 p+1}\right)=J_{0} \partial\left[s^{\prime} x u^{2 p+1}\right]=J_{0}\left[(1-\tau) s^{\prime} x u^{2 p}\right]=(1-\tau) s^{\prime} x u^{p} .
$$

It then follows that $\tilde{J}=J$. We also have $h \partial I_{0}\left(x u^{0}\right)=h \partial\left(x u^{0}\right)=0$. If $p \geqslant 1$, then $h \partial I_{0}\left(x u^{p}\right)=$ $h \partial\left(x u^{p}\right)=0$. If $p \geqslant 1$, then

$$
h \partial I_{0}\left(x u^{p}\right)=h \partial\left(x u^{2 p}\right)=h\left(N x u^{2 p-1}\right)=s^{\prime} N x u^{2 p-1} .
$$

This shows that $h \partial I_{0}=s^{\prime} N u^{-1} I_{0} u^{-1}$, and so $\tilde{I}=(1+h \partial) I_{0}=\left(1+s^{\prime} N u^{-1}\right) I_{0}=I$.

We also have $J_{0} \partial I_{0}\left(x u^{p}\right)=J_{0} \partial\left(x u^{2 p}\right)=J_{0}\left(N x u^{2 p-1}\right)=0$, and so $J_{0} \partial I_{0}=0$. In addition, we have $J_{0} \partial h \partial I_{0}\left(x u^{0}\right)=J_{0} \partial h \partial\left(x u^{0}\right)=0=B u^{-1}\left(x u^{0}\right)$. If $p \geqslant 1$, then by (8.7) we have $h \partial I_{0}\left(x u^{p}\right)=$ $s^{\prime} N x u^{2 p-1}$, and so we get

$$
J_{0} \partial h \partial I_{0}\left(x u^{p}\right)=J_{0} \partial\left(s^{\prime} N x u^{2 p-1}\right)=J_{0}\left[(1-\tau) s^{\prime} N x u^{2 p-1}\right]=B x u^{p-1} .
$$

Therefore, we see that $J_{0} \partial h \partial I_{0}=B u^{-1}$. Thus,

$$
\tilde{\partial}=J_{0}(\partial+\partial h \partial) I_{0}=J_{0} \partial I_{0}+J_{0} \partial h \partial I_{0}=B u^{-1} .
$$

The proof is complete.

We are now in a position to prove the main result of this section.

Proposition 8.3. Let $C=(C \bullet, d, s, t)$ be an $H$-unital para-precyclic $k$-module.

(1) The maps $I: C_{\bullet}^{\natural} \rightarrow C_{\bullet}^{\natural \natural}$ and $J: C_{\bullet}^{\natural \natural} \rightarrow C_{\bullet}^{\natural}$ given by 8.4)-(8.5) are $S$-maps. 
(2) We have

$$
J I=1, \quad I J=1+(\partial+\delta) h+h(\partial+\delta) .
$$

This provides us with an $S$-deformation retract of $C^{\text {घh }}$ to $C^{\natural}$.

(3) The chain homotopy $h$ is special (i.e., $J h=0, h I=0$, and $h^{2}=0$ ).

Proof. It follows from Lemma 7.1 and Lemma 8.2 that $I$ and $J$ are chains maps giving rise to the deformation retract (8.8). Moreover, the chain homotopy $h$ is special, in the sense that $J h=0, h I=0$, and $h^{2}=0$. As mentioned above, the chain homotopy $h$ is compatible with the

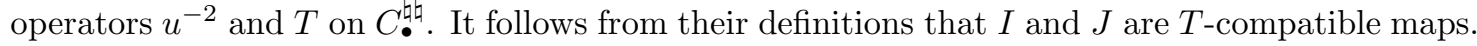
Moreover, it can be checked that $I u^{-1}=u^{-2} I$ and $J u^{-2}=u^{-1} J$. Therefore, the maps $I$ and $J$ are $S$-maps and the deformation retract (8.8) is an $S$-deformation retract of $C^{\text {घh }}$ to $C^{\natural}$. The proof is complete.

Remark 8.4. When $C$ is an $H$-unital precyclic module Proposition 8.3 was proved by Kassel 34].

Remark 8.5. It was shown by Loday-Quillen [43] that the chain map $I: C_{\bullet}^{\natural} \rightarrow C_{\bullet}^{\natural \natural}$ given by (8.4) is a quasi-isomorphism when $C$ is the cyclic module of an unital $k$-algebra and $k$ is commutative.

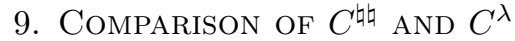

When $C$ is a precyclic $k$-module with $k \supset \mathbb{Q}$, Kassel 34 used a version of the basic perturbation lemma to construct a deformation retract of the chain complex $C^{\text {如 }}$ to Connes' cyclic complex $C^{\lambda}$. In this section, we seek for a similar result for para-precyclic modules by using the perturbation theory of Section 7 In particular, this approach avoids using the cyclic relation $\tau^{m+1}=1$, which is used in [34, but is not available in general with para-precyclic modules.

Recall that if $C=\left(C_{\bullet}, d, t\right)$ is a precyclic $k$-module, then its cyclic chain complex in the sense of Connes [7, 8, 9] is $C^{\lambda}=\left(C_{\bullet}^{\lambda}, b\right)$, where

$$
C_{m}^{\lambda}=C_{m} / \operatorname{ran}(1-\tau), \quad m \geqslant 0 .
$$

Here $\tau$ is given by (5.3) and the differential $b$ is induced from the Hochschild differential $b: C_{\bullet} \rightarrow$ $C_{\bullet-1}$. Indeed, as $b(1-\tau)=(1-\tau) b^{\prime}$ this operator descends to a unique $k$-linear differential $b: C_{\bullet}^{\lambda} \rightarrow C_{\bullet-1}^{\lambda}$. More generally, if $C=(C \bullet, d, t)$ is any para-precyclic $k$-module, then we can define the $k$-modules $C_{m}^{\lambda}, m \geqslant 0$, as in (9.1). As we still have the relation $b(1-\tau)=(1-\tau) b^{\prime}$, the Hoschschild differential descends to a $k$-linear differential $b: C_{\bullet}^{\lambda} \rightarrow C_{\bullet-1}^{\lambda}$, and so we get a chain complex $C^{\lambda}:=\left(C_{\bullet}^{\lambda}, b\right)$.

In what follows, we assume we are given a para-precyclic $k$-module $C=(C \cdot, d, t)$. We let

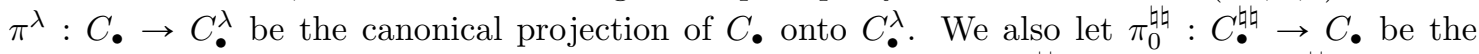
projection onto the zeroth degree component $C_{\bullet} u^{0}=C_{\bullet}$. That is, $\pi_{0}^{\text {घh }}\left(x u^{0}\right)=x$ and $\pi_{0}^{\text {घh }}\left(x u^{p}\right)=0$

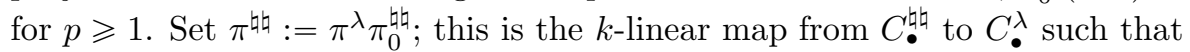

$$
\pi^{\text {虾 }}\left(x u^{0}\right)=x^{\lambda}, \quad \pi^{\text {虾 }}\left(x u^{p}\right)=0, \quad p \geqslant 1 .
$$

Lemma 9.1. We have

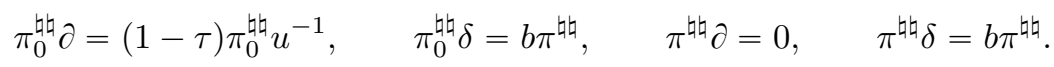

In particular, the projection $\pi^{\text {妕 }}: C^{\text {妕 }} \rightarrow C^{\lambda}$ is a chain map.

Proof. Let $x \in C_{\text {. . If }} p \geqslant 2$, then $\partial\left(x u^{p}\right)$ and $u^{-1}\left(x u^{p}\right)=x u^{p-1}$ are both contained in $\oplus_{q \geqslant 1} C \cdot u^{q}=$ ker $\pi_{0}^{\text {घh }}$, and so $\pi_{0}^{\text {吅 }} \partial\left(x u^{p}\right)=0=(1-\tau) \pi_{0}^{\text {吅 }} u^{-1}\left(x u^{p}\right)$. As $\partial\left(x u^{0}\right)=0=u^{-1}\left(x u^{0}\right)$ we also have $\pi_{0}^{\text {如 }} \partial\left(x u^{0}\right)=0=(1-\tau) \pi_{0}^{\text {घh }} u^{-1}\left(x u^{0}\right)$. In addition, by definition $\partial(x u)=(1-\tau) x u^{0}$, and so

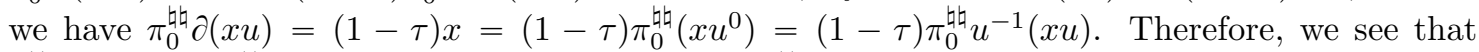

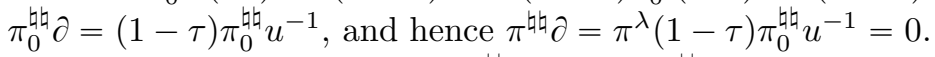

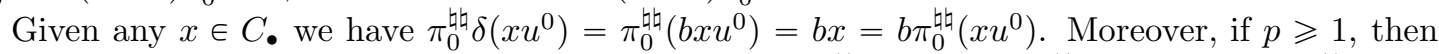

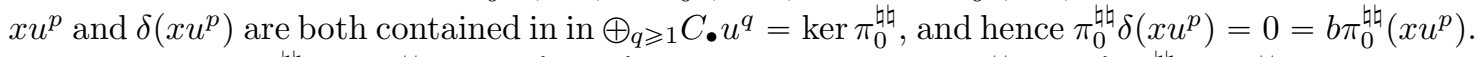

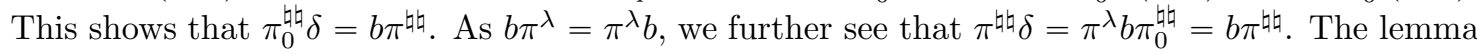
is proved. 
From now on we assume that $k \supset \mathbb{Q}$. In what follows, given $x \in C_{m}, m \geqslant 0$, we set

$$
\hat{x}=(m+1)^{-1} x \quad \text { and } \quad \hat{N} x=N \hat{x} .
$$

We then let $\nu: C \bullet \rightarrow C_{\bullet}^{\text {虾 }}$ be the $k$-linear map defined by

$$
\nu(x)=\hat{N} x u^{0}, \quad x \in C_{\bullet} .
$$

For $j \geqslant 0$, set $N_{j}(X)=\sum_{\ell \leqslant j} X^{\ell} \in k[X]$. Note that $X^{j+1}-1=(X-1) N_{j}(X)$. In addition, let $D_{m}(X) \in k[X]$ be the polynomial given by

$$
D_{m}(X)=\sum_{0 \leqslant j \leqslant m}(m-j) X^{j}=\sum_{0 \leqslant j \leqslant m-1} N_{j}(X) .
$$

We observe that

$$
N_{m}(X)-(m+1)=\sum_{0 \leqslant j \leqslant m}\left(X^{j}-1\right)=\sum_{1 \leqslant j \leqslant m}(X-1) N_{j-1}(X)=(X-1) D_{m}(X) .
$$

Let $\hat{D}: C \bullet \rightarrow C$ • be the $k$-linear map defined by

$$
\hat{D} x:=D_{m}(\tau) \hat{x}=\sum_{0 \leqslant j \leqslant m}(m-j) \tau^{j} \hat{x}, \quad x \in C \bullet .
$$

Note that by (9.3) we have

$$
\hat{N}+(1-\tau) \hat{D}=1
$$

We then let $\varphi: C_{\bullet}^{\text {吅 }} \rightarrow C_{\bullet}^{\text {吅 }}$ be the $k$-linear map defined by

$$
\varphi\left(x u^{2 p}\right)=-\hat{D} x u^{2 p+1}, \quad \varphi\left(x u^{2 p+1}\right)=-\hat{x} u^{2 p+2}, \quad x \in C_{\bullet} .
$$

Lemma 9.2 (compare [34]). We have

$$
\partial \nu=0, \quad \pi_{0}^{\text {仲 }} \nu=\hat{N}, \quad \nu \pi_{0}^{\text {仲 }}=1+\partial \varphi+\varphi \partial .
$$

Proof. As the range of $\nu$ is contained in $C \bullet u^{0} \subset \operatorname{ker} \partial$, we have $\partial \nu=0$. Let $x \in C \bullet$. We have $\pi_{0}^{\text {吅 }} \nu(x)=\pi_{0}^{\text {仲 }}\left(\hat{N} x u^{0}\right)=\hat{N} x$, and so $\pi_{0}^{\text {叫 }} \nu=\hat{N}$. By using (9.5) we also see that $(1+\varphi \partial+\partial \varphi)\left(x u^{0}\right)$ is equal to

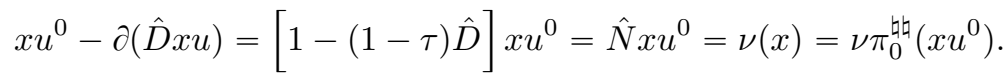

If $p \geqslant 1$, then $(\varphi \partial+\partial \varphi)\left(x u^{2 p}\right)$ is equal to

$$
\varphi\left(N x u^{2 p-1}\right)-\partial\left(\hat{D} x u^{2 p+1}\right)=-(\hat{N}+(1-\tau) \hat{D}) x u^{2 p}=-x u^{2 p} .
$$

This gives $(1+\varphi \partial+\partial \varphi)\left(x u^{2 p}\right)=0=\nu \pi_{0}^{\text {叫 }}\left(x u^{2 p}\right)$. In addition, if $p \geqslant 0$, then $(\varphi \partial+\partial \varphi)\left(x u^{2 p+1}\right)$ is equal to

$$
\varphi\left[(1-\tau) x u^{2 p}\right]-\partial\left(\hat{x} u^{2 p+1}\right)=-((1-\tau) \hat{D}+\hat{N}) x u^{2 p+1}=-x u^{2 p+1} .
$$

Thus, as above, we have $(1+\varphi \partial+\partial \varphi)\left(x u^{2 p+1}\right)=0=\nu \pi_{0}^{\text {吅 }}\left(x u^{2 p+1}\right)$. All this shows that $1+\varphi \partial+\partial \varphi$ agrees with $\nu \pi_{0}^{\text {仲 }}$ on all $C_{\bullet}^{\text {如 }}$. The proof is complete.

As it turns out, Lemma 9.1 and Lemma 9.2 allows us to apply Lemma 7.7 to the para-twin

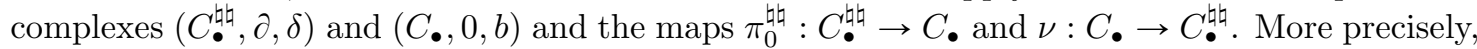
in the notation of Section 7 the $\Delta$-operator of $\left(C_{\bullet}, 0, b\right)$ is zero and it follows from Lemma 6.1 that the $\Delta$-operator of $\left(C_{\bullet}^{\natural}, \partial, \delta\right)$ is zero as well. In addition, by Lemma 9.1 and Lemma 9.2 the map $\pi_{0}^{\text {叫 }}$ is compatible with the $\delta$-operators, the map $\nu$ is compatible with the $\partial$-operators and $\pi_{0} \varphi=0$. Therefore, the assumptions of Lemma 7.7 are fulfilled.

Let $\varphi^{\text {仲 }}: C_{\bullet}^{\text {仲 }} \rightarrow C_{\bullet+1}^{\text {仲 }}$ and $\nu^{\text {耴 }}: C_{\bullet}^{\lambda} \rightarrow C_{\bullet}^{\text {仲 }}$ be the $k$-linear maps defined by

$$
\varphi^{\text {仲 }}=\sum_{j \geqslant 0}(\varphi \delta)^{j} \varphi \quad \text { and } \quad \nu_{0}^{\text {仲 }}=\left(1+\varphi^{\text {耴 }} \delta\right) \nu^{\lambda}=\sum_{j \geqslant 0}(\varphi \delta)^{j} \nu .
$$


We also introduce the $k$-linear map $\mu^{\text {印 }}: C \bullet \rightarrow C_{\bullet}^{\text {印 }}$ given by

$$
\mu^{\text {仲 }}(x)=\sum_{j \geqslant 0}(\varphi \delta)^{j}\left(\hat{x} u^{0}\right), \quad x \in C_{\bullet} .
$$

Note that $\nu_{0}^{\text {吅 }}(x)=\mu^{\text {妕 }}(N x)$, since $\nu(x)=N \hat{x} u^{0}$. In addition, for $x \in C_{m}$, we set $\hat{b}^{\prime} x=m^{-1} b x$, with the convention that $\hat{b}^{\prime} x=0$ when $m=0$.

Lemma 9.3. Let $C$ be a para-precyclic $k$-module, and assume that $k \supset \mathbb{Q}$.

(1) We have

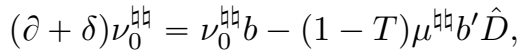

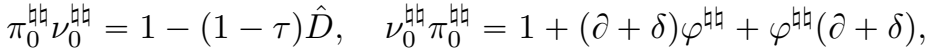

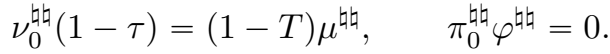

(2) For all $x \in C_{\bullet}$, we have

$$
\nu_{0}^{\text {叫 }}(x)=\sum_{j \geqslant 0}(-1)^{j}(1-\hat{D} b u)\left(\hat{b}^{\prime} \hat{D} b\right)^{j} \hat{N} x u^{2 j} .
$$

Proof. In the current setup (7.22) and (7.24) give

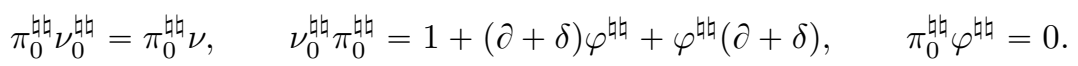

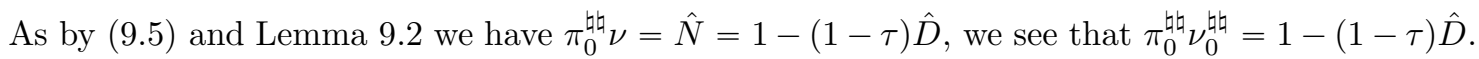
Thus, in the notation of (7.23) we have

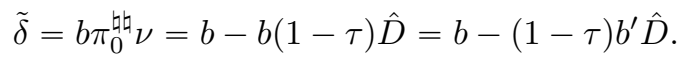

We also observe that

$$
\nu_{0}^{\natural \natural}(1-\tau)=\mu^{\natural \natural} N(1-\tau)=\mu^{\natural \natural}(1-T)=(1-T) \mu^{\natural \natural} .
$$

Therefore, the chain map property (17.21) gives

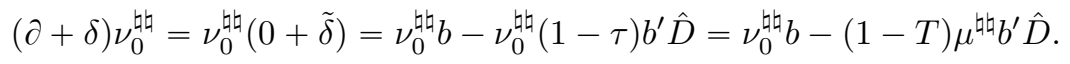

It remains to prove (9.11). Let $x \in C_{\bullet}$. We have

$$
\begin{gathered}
(\varphi \delta)\left(x u^{2 p}\right)=\varphi\left(b x u^{2 p+1}\right)=-\hat{D} b x u^{2 p+1}, \\
(\varphi \delta)\left(x u^{2 p+1}\right)=-\varphi\left(b^{\prime} x u^{2 p+1}\right)=\hat{b}^{\prime} x u^{2 p+2} .
\end{gathered}
$$

An induction then shows, for all $j \geqslant 0$, we have

$$
(\varphi \delta)^{2 j}\left(x u^{0}\right)=(-1)^{j}\left(\hat{b}^{\prime} \hat{D} b\right)^{j} x u^{2 j}, \quad(\varphi \delta)^{2 j+1}\left(x u^{0}\right)=(-1)^{j+1} \hat{D} b\left(\hat{b}^{\prime} \hat{D} b\right)^{j} x u^{2 j+1} .
$$

Thus,

$$
\mu^{\text {घҺ }}(x)=\sum_{j \geqslant 0}(\varphi \delta)^{j}\left(\hat{x} u^{0}\right)=\sum_{j \geqslant 0}(-1)^{j}(1-\hat{D} b u)\left(\hat{b}^{\prime} \hat{D} b\right)^{j} \hat{x} u^{2 j} .
$$

As $\nu_{0}^{\text {घদ }}=\mu^{\natural \natural} N$ we obtain (9.11). The proof is complete.

Remark 9.4. For future purpose we record the following formulas for the chain homotopy $\varphi^{\text {th }}$ that we get from (9.12)- (9.13). Namely, for all $x \in C_{\bullet}$, we have

$$
\begin{gathered}
\varphi^{\natural \natural}\left(x u^{2 p}\right)=\sum_{j \geqslant 0}(-1)^{j+1}\left(1+\hat{b}^{\prime} u\right)\left(\hat{D} b \hat{b}^{\prime}\right)^{j} \hat{D} x u^{2 p+2 j+1}, \\
\varphi^{\natural \natural}\left(x u^{2 p+1}\right)=\sum_{j \geqslant 0}(-1)^{j+1}(1-\hat{D} b u)\left(\hat{b}^{\prime} \hat{D} b\right)^{j} \hat{x} u^{2 p+2 j+2} .
\end{gathered}
$$


As $R_{\bullet}^{T} \subset \operatorname{ran}(1-\tau)$, the chain map $\pi^{\text {仲 }}: C_{\bullet}^{\text {仲 }} \rightarrow C_{\bullet}^{\lambda}$ descends to a unique $k$-linear chain map

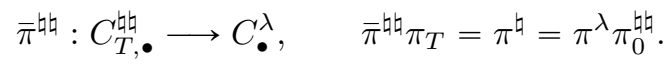

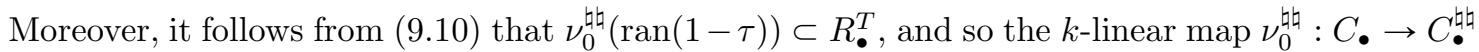
also descends to a $k$-linear map,

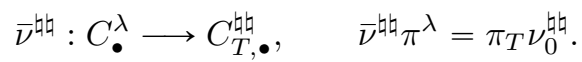

It also follows from (9.16) (9.17) that the homotopy $\varphi^{\text {th }}$ is compatible with the $T$-operators.

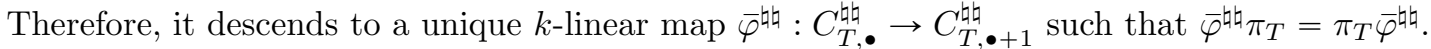

Proposition 9.5. Suppose that $C$ is a para-precyclic $k$-module and $k \supset \mathbb{Q}$. Then the $k$-linear map $\bar{\nu}^{\text {如 }}: C_{\bullet}^{\lambda} \rightarrow C_{T, \bullet}^{\text {如 }}$ is a chain map such that

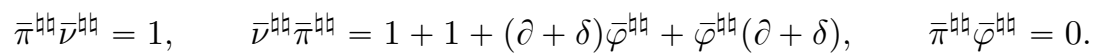

In particular, we obtain a deformation retract of $C_{T}^{\text {姉 }}$ to $C^{\lambda}$.

Proof. We observe that $\left[(\partial+\delta) \bar{\nu}^{\text {虲 }}-\bar{\nu}^{\text {घh }} b\right] \pi^{\lambda}$ is equal to

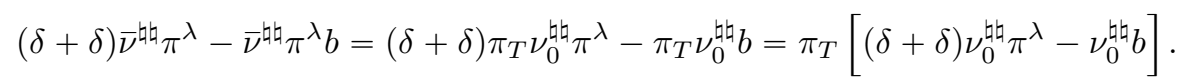

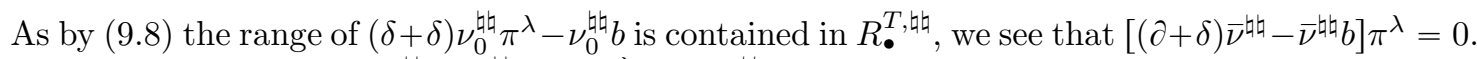

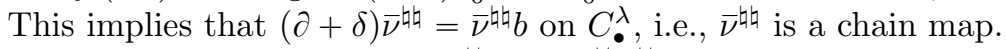

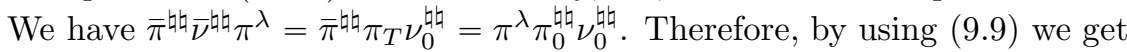

$$
\bar{\pi}^{\natural \natural} \bar{\nu}^{\natural \natural} \pi^{\lambda}=\pi^{\lambda}[1-(1-\tau) \hat{D}]=\pi^{\lambda} .
$$

It then follows that $\bar{\pi}^{\natural \natural} \bar{\nu}^{\natural \natural} \pi^{\lambda}=1$ on on $C_{\bullet}^{\lambda}$.

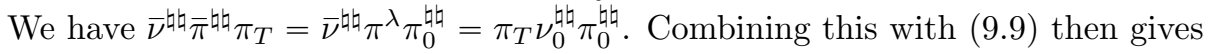

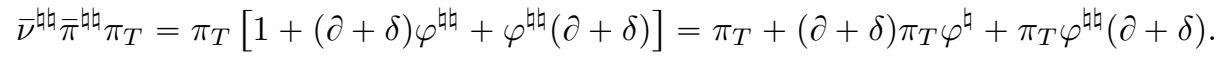

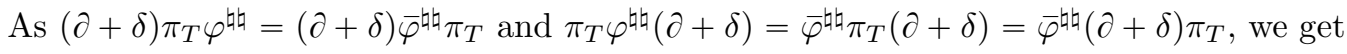

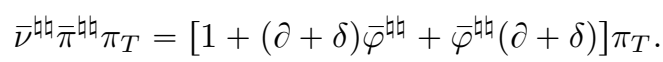

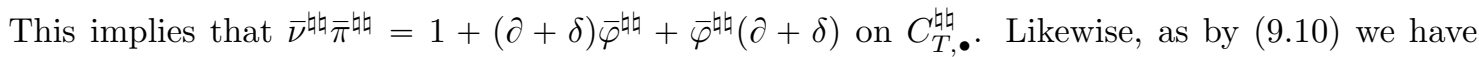

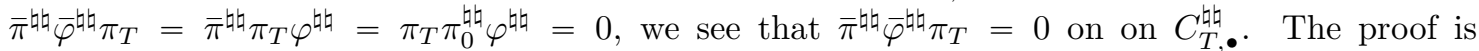
complete.

It is worth specializing Proposition 9.5 to precyclic $k$-modules. In this case $\bar{\pi}^{\text {th }}$ is just the

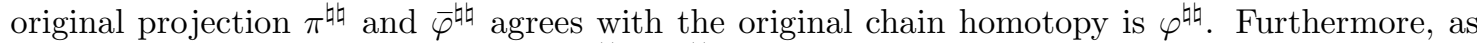
$T=1$ the equality (9.8) gives $(\partial+\delta) \nu_{0}^{\text {घh }}=\nu_{0}^{\text {蚂 }} b$. Thus, in the precyclic case it is immediate that $\nu_{0}^{\text {蚛 }}$ descends to a unique $k$-linear chain map,

$$
\nu^{\text {吅 }}: C_{\bullet}^{\lambda} \longrightarrow C_{\bullet}^{\text {呺 }}, \quad \nu^{\text {吅 }} \pi^{\lambda}=\nu_{0}^{\text {吅 }} .
$$

Therefore, we arrive at the following statement.

Corollary 9.6 (see also 34]). Suppose that $C$ is a precyclic $k$-module and $k \supset \mathbb{Q}$. Then we have

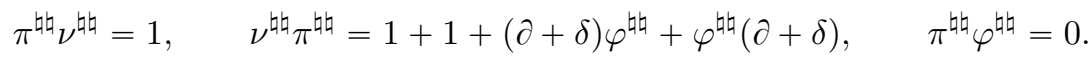

In particular, this provides us with a deformation retract of $C^{\text {th }}$ to $C^{\lambda}$.

Remark 9.7. When $C$ is a precyclic module it is well known that the projection $\pi^{\text {印 }}: C_{\bullet}^{\text {蚂 }} \rightarrow C_{\bullet}^{\lambda}$ is a quasi-isomorphism [8] (see also [42, 43]).

Remark 9.8. When $C$ is a precyclic $k$-module we recover the deformation retract of $C^{\text {th }}$ to $C^{\lambda}$ produced by Kassel [34] as follows. For $m \geqslant 0$ set $\tilde{D}_{m}(X)=\sum_{j=0}^{m} j X^{j} \in k[X]$, and let $\check{D}: C$ • $\rightarrow$ $C$. be the $k$-linear map defined by

$$
\check{D} x=\tilde{D}_{m}(\tau) \hat{x}, \quad x \in C_{m}, m \geqslant 0 .
$$


We have $\tilde{D}_{m}(\tau)=\sum_{j=0}^{m}(m-j) \tau^{m-j}=\tau^{m} D_{m}\left(\tau^{-1}\right)$. By combining this with (9.5) and the equality $\tau^{m+1}=1$, it can be shown that, when $C$ is precyclic, we have

$$
\hat{N}-(1-\tau) \check{D}=1 \text {. }
$$

Therefore, in the precyclic case, we obtain an alternative chain homotopy satisfying (9.21) by substituting $-\check{D}$ for $\hat{D}$ in the formula (9.6) for $\varphi$. This gives back the chain homotopy used by Kassel [34]. We then recover his formula for the homotopy inverse of $\pi^{\text {如 }}$ by substituting $-\check{D}$ for $\hat{D}$ in the formula (9.11).

Suppose that $C$ is a quasi-precyclic $k$-module, so that $C_{\bullet}=C_{\bullet}^{T} \oplus R_{\bullet}^{T}$. Let $\pi^{T}: C_{\bullet} \rightarrow C_{\bullet}$ be the projection onto $C_{\bullet}^{T}$ defined by this splitting. This is a para-precyclic $k$-module map, and so it

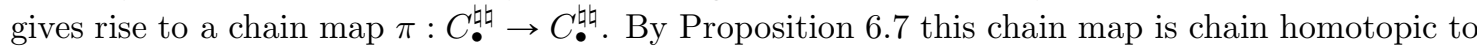

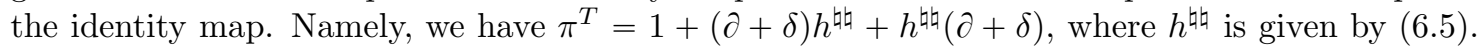
We observe that (9.10) implies that $\pi^{T} \nu_{0}^{\text {吼 }}(1-\tau)=\pi^{T}(1-T) \mu^{\text {叫 }}=0$, and so $\pi^{T} \nu_{0}^{\text {घ曰 }}$ descends to a unique $k$-linear map,

$$
\nu^{T, \text { 如 }}: C_{\bullet}^{\lambda} \longrightarrow C_{\bullet}^{\text {如 }}, \quad \nu^{T, \text { 如 }} \pi^{\lambda}=\pi^{T} \nu_{0}^{\text {幼 }} .
$$

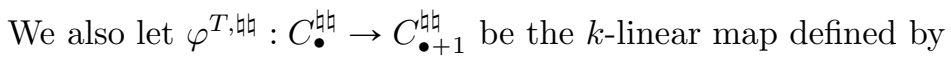

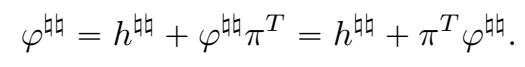

Proposition 9.9. Assume that $C$ is a quasi-precyclic $k$-module and $k \supset \mathbb{Q}$. Then the $k$-linear

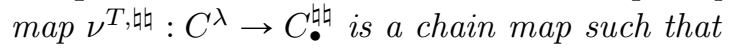

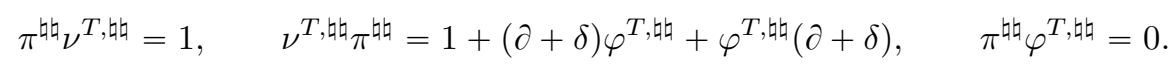

In particular, we obtain a deformation retract of $C^{\text {th }}$ to $C^{\lambda}$.

Proof. Like the canonical projection $\pi_{T}: C_{\bullet} \rightarrow C_{T, \bullet}$, the projection $\pi^{T}$ is a para-precyclic $k$-linear map that is annihilated by $R_{\bullet}^{T}$. Thus, by using the equality $\nu^{T, \text { 吅 }} \pi^{\lambda}=\pi^{T} \nu_{0}^{\text {घ }}$ and arguing along similar lines as that of the proof of Proposition 9.5 it can be shown that $\nu^{T \text {, 蚂 }}: C^{\lambda} \rightarrow C_{\bullet}^{\text {如 }}$ is a chain map. In addition, as $\pi^{\natural \natural} \pi^{T}=\pi^{\text {机 }}$, in the same way as in (9.19) we have

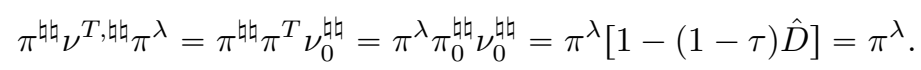

Therefore, we see that $\pi^{\text {如}} \nu^{T, \text { 䗉 }}=1$ on $C_{\bullet}^{\lambda}$.

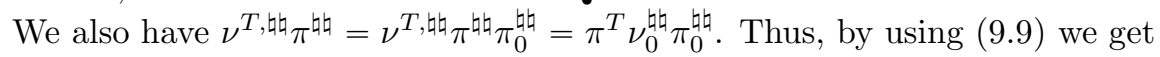

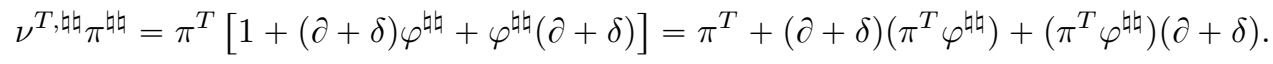

Combining this with the homotopy formula $\pi^{T}=1+(\partial+\delta) h^{\text {घh }}+h^{\text {घh }}(\partial+\delta)$ then gives

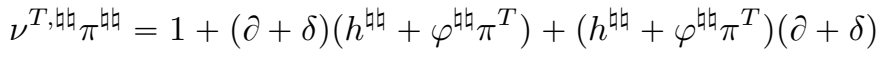

$$
\begin{aligned}
& =1+(\partial+\delta) \varphi^{T, \text { 蜡 }}+\varphi^{T, \text { 虬 }}(\partial+\delta) .
\end{aligned}
$$

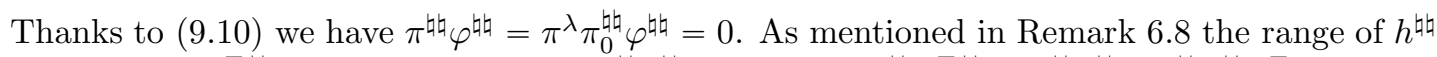

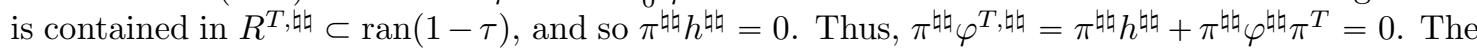
proof is complete.

\section{Comparison of $C^{\natural}$ And $C^{\lambda}$}

When $C$ is a precyclic $k$-module it is well known that if $k \supset \mathbb{Q}$, then the chain complexes $C^{\natural}$ and $C^{\lambda}$ are quasi-isomorphic 7, 8, 9] (see also 43). In this section, we shall combine the results of the previous two sections to compare $C^{\natural}$ and $C^{\lambda}$ in the case of $H$-unital para-precyclic modules.

Throughout this section we assume that $k \supset \mathbb{Q}$, and let $C=(C \cdot, d, s, t)$ be an $H$-unital preparacyclic $k$-module. Let $\pi_{0}: C_{\bullet}^{\natural} \rightarrow C$ • be the natural projection onto the zeroth degree summand $C_{\bullet} u^{0}=C_{\bullet}$. By composing it with the projection $\pi^{\lambda}: C_{\bullet} \rightarrow C_{\bullet}^{\lambda}$ we get natural projection $\pi^{\natural}:=\pi^{\lambda} \pi_{0}^{\natural}: C_{\bullet}^{\natural} \rightarrow C_{\bullet}^{\lambda}$. Thus,

$$
\pi^{\natural}\left(x u^{0}\right)=x^{\lambda}, \quad \pi_{35}^{\natural}\left(x u^{p}\right)=0, \quad p \geqslant 1 .
$$


Note that $\pi_{0}^{\natural}=\pi_{0}^{\natural \natural} I$ and $\pi^{\natural}=\pi^{\natural \natural} I$. We also observe that

$$
\pi_{0}^{\natural} b=b \pi_{0}^{\natural}, \quad \pi_{0}^{\natural} B=B \pi_{0}^{\natural}, \quad \pi^{\natural} b=b \pi^{\natural}, \quad \pi^{\natural} B=0 .
$$

In particular, this implies that $\pi^{\natural}: C_{\bullet}^{\natural} \rightarrow C_{\bullet}^{\lambda}$ is a chain map.

Let $\nu_{0}^{\natural}: C_{\bullet} \rightarrow C_{\bullet}^{\natural}$ and $\varphi^{\natural}: C_{\bullet}^{\natural} \rightarrow C_{\bullet}$ be the $k$-linear maps defined by

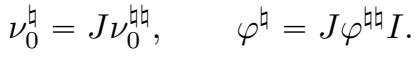

We also set $\mu^{\natural}=J \mu^{\natural \natural}$, where $\mu^{\natural \natural}: C \bullet \rightarrow C^{\text {仲 }}$ is given by (9.15).

Lemma 10.1. Suppose that $C$ is an $H$-unital para-precyclic $k$-module and $k \supset \mathbb{Q}$.

(1) We have

$$
\begin{gathered}
\left(b+B u^{-1}\right) \nu_{0}^{\natural}=\nu_{0}^{\natural} b-(1-T) \mu^{\natural} b^{\prime} \hat{D}, \\
\pi_{0}^{\natural} \nu_{0}^{\natural}=1-(1-\tau)\left[1+s^{\prime} \hat{D} b \hat{N}\right], \\
\nu_{0}^{\natural} \pi_{0}^{\natural}=1+\left(b+B u^{-1}\right) \varphi^{\natural}+\varphi^{\natural}\left(b+B u^{-1}\right), \\
\nu_{0}^{\natural}(1-\tau)=(1-T) \mu^{\natural}, \quad \pi_{0}^{\natural} \varphi^{\natural}=-(1-\tau) s^{\prime} \hat{b} \hat{D} \pi_{0}^{\natural} .
\end{gathered}
$$

(2) For all $x \in C_{\bullet}$, we have

$$
\nu_{0}^{\natural}(x)=\sum_{j \geqslant 0}(-1)^{j}\left[1-(1-\tau) s^{\prime} \hat{D} b\right]\left(\hat{b}^{\prime} \hat{D} b\right)^{j} \hat{N} x u^{j} .
$$

Proof. By (9.10) we have $\nu_{0}^{\natural}(1-\tau)=J \nu_{0}^{\natural \natural}(1-\tau)=J(1-T) \mu^{\natural \natural}=(1-T) \mu^{\natural}$. As $J$ is a chain map we also have $\left(b+B u^{-1}\right) \nu_{0}^{\natural}=\left(b+B u^{-1}\right) J \nu_{0}^{\text {仲 }}=J(\partial+\delta) \nu_{0}^{\text {叫 }}$. Combining this with (9.8) then gives

$$
\left(b+B u^{-1}\right) \nu_{0}^{\natural}=J \nu_{0}^{\text {印 }} b-(1-T) J \mu^{\natural \natural} b^{\prime} D=\nu_{0}^{\natural} b-(1-T) \mu^{\natural} b^{\prime} \hat{D} .
$$

Moreover, by using (9.9) we see that $\nu_{0}^{\natural} \pi_{0}^{\natural}$ is equal to

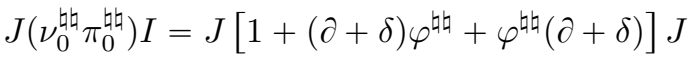

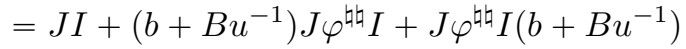

$$
\begin{aligned}
& =1+\left(b+B u^{-1}\right) \varphi^{\natural}+\varphi^{\natural}\left(b+B u^{-1}\right) \text {. }
\end{aligned}
$$

It follows from the formulas (8.5) and (9.11) for $J$ and $\nu_{0}^{\text {th }}$ that, given any $x \in C_{\bullet}$, we have

$$
\begin{aligned}
\nu_{0}^{\natural}(x) & =\sum_{j \geqslant 0}(-1)^{j} J\left[\left(\hat{b}^{\prime} \hat{D} b\right)^{j} \hat{N} x u^{2 j}-\hat{D} b\left(\hat{b}^{\prime} \hat{D} b\right)^{j} \hat{N} x u^{2 j+1}\right] \\
& =\sum_{j \geqslant 0}(-1)^{j}\left[1-(1-\tau) s^{\prime} \hat{D} b\right]\left(\hat{b}^{\prime} \hat{D} b\right)^{j} \hat{N} u^{j} .
\end{aligned}
$$

This proves (10.7). Combining this with the equality $\hat{N}+(1-\tau) \hat{D}=1$ further gives

$$
\pi_{0} \nu_{0}^{\natural}=\hat{N}-(1-\tau) s^{\prime} \hat{D} b \hat{N}=1-(1-\tau)\left[\hat{D}+s^{\prime} \hat{D} b \hat{N}\right] .
$$

We have $\pi_{0}^{\natural} \varphi^{\natural}=\pi_{0}^{\natural} J \varphi^{\natural \natural} I$. It follows from (8.5) that we have

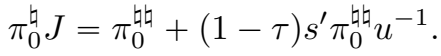

Let $x \in C_{\bullet}$. The formulas 9.16) -9.17) implies that $\varphi^{\text {仲 }}\left(x u^{p}\right)$ is contained in $\oplus_{q \geqslant p+1} C \bullet u^{q}$, and so $\varphi^{\natural}$ 蛣 $\left(x u^{p}\right)=0$ when $p \geqslant 1$. As (9.6) shows that $\varphi\left(x u^{0}\right)=-\hat{D} x u \bmod \oplus_{q \geqslant 2} C \bullet u^{q}$, we also get

$$
\pi_{0}^{\natural} J \varphi^{\text {吅 }}\left(x u^{0}\right)=-\pi_{0}^{\text {仲 }} J(\hat{D} x u)=-(1-\tau) s^{\prime} \hat{D} x=-(1-\tau) \hat{D} \pi_{0}^{\text {仲 }}\left(x u^{0}\right) .
$$

Therefore, we see that $\pi_{0}^{\natural} J \varphi^{\natural \natural}=-(1-\tau) \hat{D} \pi_{0}^{\natural \natural}$. As $\pi_{0}^{\natural \natural} I=\pi_{0}^{\natural}$, we then obtain

$$
\pi_{0}^{\natural} \varphi^{\natural}=\pi_{0}^{\natural} J \varphi^{\natural \natural} I=-(1-\tau) \hat{D} s^{\prime} \pi_{0}^{\natural \natural} I=-(1-\tau) \hat{D} \pi_{0}^{\natural} .
$$

The proof is complete. 
Let $\pi_{T}: C_{\bullet} \rightarrow C_{T}, \bullet$ be the canonical projection of $C_{\bullet}$ onto $C_{T, \bullet}$. As this is a map of $H$-unital para-precyclic $k$-modules, it gives rise to a chain map $\pi_{T}: C^{\natural} \rightarrow C_{T, \bullet}^{\natural}$. Therefore, in the same way as with the projection $\pi^{\natural \natural}$ above, the projection $\pi^{\natural}: C_{\bullet}^{\natural} \rightarrow C_{\bullet}^{\lambda}$ descends to a unique $k$-linear chain map,

$$
\bar{\pi}^{\natural}: C_{T, \bullet}^{\natural} \longrightarrow C_{\bullet}^{\lambda}, \quad \bar{\nu}^{\natural} \pi^{\lambda}=\pi_{T} \pi^{\natural} .
$$

It follows from (10.6) that $\pi_{T} \nu_{0}^{\natural}(1-\tau)=\pi_{T}(1-T) \mu^{\natural}=0$. Therefore, the $k$-linear map $\pi_{T} \nu_{0}^{\natural}: C \bullet \bullet C_{T, \bullet}^{\natural}$ descends to a unique $k$-linear map,

$$
\bar{\nu}^{\natural}: C_{\bullet}^{\lambda} \longrightarrow C_{T, \bullet}^{\natural}, \quad \bar{\nu}^{\natural} \pi^{\lambda}=\pi_{T} \nu_{0}^{\natural} .
$$

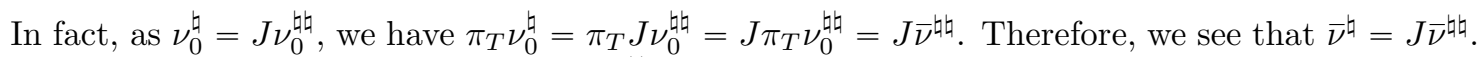
In particular, as $J$ is a chain map, and $\bar{\nu}^{\text {妁 }}$ is a chain map as well by Proposition 9.5, we see that $\bar{\nu}^{\natural}: C_{\bullet}^{\lambda} \rightarrow C_{T, \bullet}^{\natural}$ is a chain map.

In addition, as the chain homotopy $\varphi^{\natural}: C_{\bullet}^{\natural} \rightarrow C_{\bullet+1}^{\natural}$ is compatible with the $T$-operator, it descends to a unique $k$-linear map $\bar{\varphi}^{\natural}: C_{T, \bullet}^{\natural} \rightarrow C_{T, \bullet+1}^{\natural}$ such that $\bar{\varphi}^{\natural \natural} \pi_{T}=\pi_{T} \varphi^{\natural}$. By using Lemma 10.1 and arguing along similar lines as that of the proof of Proposition 9.5 we then obtain the following result.

Proposition 10.2. Suppose that $C$ is an $H$-unital para-precyclic $k$-module and $k \supset \mathbb{Q}$. Then

$$
\bar{\pi}^{\natural} \bar{\nu}^{\natural}=1, \quad \bar{\nu}^{\natural} \bar{\pi}^{\natural}=1+\left(b+B u^{-1}\right) \bar{\varphi}^{\natural}+\bar{\varphi}^{\natural}\left(b+B u^{-1}\right), \quad \bar{\pi}^{\natural} \bar{\varphi}^{\natural}=0 .
$$

In particular, we obtain a deformation retract of $C_{T}^{\natural}$ to $C^{\lambda}$.

Proof. By using Lemma 10.1 and arguing along similar lines as that of the proof of Proposition 9.5 it can be shown that $\bar{\pi}^{\natural} \bar{\nu}^{\natural}=1$ and $\bar{\nu}^{\natural} \bar{\pi}^{\natural}=1+\left(b+B u^{-1}\right) \bar{\varphi}^{\natural}+\bar{\varphi}^{\natural}\left(b+B u^{-1}\right)$. Moreover, by using (10.6) we see that $\bar{\pi}^{\natural} \bar{\varphi}^{\natural} \pi_{T}=\bar{\pi}^{\natural} \pi_{T} \varphi^{\natural}=\pi^{\lambda} \pi_{0}^{\natural} \varphi^{\natural}=-\pi^{\lambda}(1-\tau) s^{\prime} \hat{b} \hat{D}=0$. It then follows that $\bar{\pi}^{\natural} \bar{\varphi}^{\natural}=0$ on $C_{T, \bullet}^{\natural}$. The proof is complete.

It is interesting to specialize Proposition 10.2 to $H$-unital precyclic $k$-modules. In this case $\bar{\pi}^{\natural}$ is just the projection $\pi^{\natural}$ and $\bar{\varphi}^{\natural}$ agrees with the chain homotopy $\varphi^{\natural}$. Furthermore, as $T=1$ the equalities (10.3) and (10.6) ensure us that $\nu_{0}^{\natural}(1-\tau)=0$ and $\nu_{0}^{\natural} b=\left(b+B u^{-1}\right) \nu_{0}^{\natural}$. Thus, it is immediate that $\nu_{0}^{\natural}$ descends to a unique $k$-linear chain map,

$$
\nu^{\natural}: C_{\bullet}^{\lambda} \longrightarrow C_{\bullet}^{\natural}, \quad \nu^{\natural} \pi^{\lambda}=\nu_{0}^{\natural} .
$$

Therefore, we obtain the following statement.

Corollary 10.3. Suppose that $C$ is an $H$-unital precyclic $k$-module and $k \supset \mathbb{Q}$. Then we have

$$
\pi^{\natural} \nu^{\natural}=1, \quad \nu^{\natural} \pi^{\natural}=1+\left(b+B u^{-1}\right) \varphi^{\natural}+\varphi^{\natural}\left(b+B u^{-1}\right), \quad \pi^{\natural} \varphi^{\natural}=0 .
$$

In particular, we obtain a deformation retract of $C^{\natural}$ to $C^{\lambda}$.

Suppose now that $C$ is an $H$-unital quasi-precyclic $k$-module, so that $C_{\bullet}=C_{\bullet}^{T} \oplus R_{\bullet}^{T}$. Let $\pi^{T}$ : $C_{\bullet} \rightarrow C_{\bullet}$ be the projection of $C_{\bullet}$ on $C_{\bullet}^{T}$ associated with this splitting. This is an $H$-unital paraprecyclic $k$-module map, and so it yields a chain map $C_{\bullet}^{\natural} \rightarrow C_{\bullet}^{\natural}$. By Proposition 3.8 this chain map is $S$-homotopy equivalent to the identity map. Namely, $\pi^{T}=1+\left(b+B u^{-1}\right) h+h\left(b+B u^{-1}\right)$, where $h: C_{\bullet}^{\natural} \rightarrow C_{\bullet+1}^{\natural}$ is given by (3.5). Note also that by (10.6) we have $\pi^{T} \nu_{0}^{\natural}(1-\tau)=\pi^{T}(1-T) \mu^{\natural}=0$, and so $\pi^{T} \nu_{0}^{\natural}$ descends to a unique $k$-linear map,

$$
\nu^{T, \natural}: C_{\bullet}^{\lambda} \longrightarrow C_{\bullet}^{\natural}, \quad \nu^{T, \natural} \pi^{\lambda}=\pi^{T} \nu_{0}^{\natural} .
$$

We also let $\varphi^{T, \natural}: C_{\bullet}^{\natural} \rightarrow C_{\bullet+1}^{\natural}$ be the $k$-linear map defined by

$$
\varphi^{T, \natural}=h+\varphi^{\natural} \pi^{T}=h+\varphi^{\natural} \pi^{T} .
$$


Proposition 10.4. Assume that $C$ is an $H$-unital quasi-precyclic $k$-module and $k \supset \mathbb{Q}$. Then the $k$-linear map $\nu^{T, \natural}: C_{\bullet}^{\lambda} \rightarrow C_{\bullet}^{\natural}$ is a chain map such that

$$
\pi^{\natural} \nu^{T, \natural}=1, \quad \nu^{T, \natural} \pi^{\natural}=1+\left(b+B u^{-1}\right) \varphi^{T, \natural}+\varphi^{T, \natural}\left(b+B u^{-1}\right), \quad \pi^{\natural} \varphi^{T, \natural}=0 .
$$

In particular, we obtain a deformation retract of $C^{\natural}$ to $C^{\lambda}$.

Proof. By using Lemma 10.1 and arguing along similar lines as that of the proof of Proposition9.9 we see that $\pi^{\natural} \nu^{T, \natural}=1$ and $\nu^{T, \natural} \pi^{\natural}=1+\left(b+B u^{-1}\right) \varphi^{T, \natural}+\varphi^{T, \natural}\left(b+B u^{-1}\right)$. As mentioned in Remark 2.13, the range of $h$ is contained in $R^{T, \natural} \subset \operatorname{ran}(1-\tau)$, and so $\pi^{\natural} h=0$. By using (10.6) we also get $\pi^{\natural} \varphi^{\natural}=\pi^{\lambda} \pi_{0}^{\natural} \varphi^{\natural}=-\pi^{\lambda}(1-\tau) s^{\prime} \hat{b} \hat{D}=0$. Therefore, we see that $\pi^{\natural} \varphi^{T, \natural}=\pi^{\natural} h+\pi^{\natural} \varphi^{\natural} \pi^{T}=0$. The proof is complete.

\section{The Periodicity Operator}

It was observed by Kassel 34 that, given any precyclic $k$-module $C$ with $k \supset \mathbb{Q}$, the deformation retract of $C^{\text {约 }}$ to Connes' complex $C^{\lambda}$ allows us to get an alternative description of the periodicity operator of Connes [7, 8, 9] in cyclic homology.

In this section, we further elaborate on Kassel's approach to the periodicity operator. We shall relate the periodicity operator to the comparison results of the previous sections between $C^{\text {th }}$ and $C^{\lambda}$ for para-precyclic modules, and between $C^{\natural}$ and $C^{\lambda}$ in the $H$-unital case. We will also give a few applications in periodic cyclic homology.

One feature of the approach of 34 is the use of a special chain homotopy in the construction of the deformation retract of $C^{\text {th }}$ and $C^{\lambda}$ in the precyclic case. A special chain homotopy need not be available in general in the setting of para-precyclic modules ( $c f$. Remark 7.6). We shall bypass this issue by using the almost chain homotopy inverse $\nu_{0}^{\text {घh }}$ of Section 9 . Not only will this allow us to proceed in the para-precyclic case, this will also lead us to a simpler formula for the periodicity operator and an equality with Connes' periodicity at the level of chains, rather than at the cyclic homology level (compare [34, 42]).

11.1. The periodicity operator on $C_{\bullet}^{\lambda}$. Suppose that $k \supset \mathbb{Q}$, and let $C$ be a (pre)cyclic $k$ module. The cyclic homology of $C$ is a module over the cyclic cohomology of $k$. By using Connes' cyclic chain complex $C^{\lambda}$, this module structure is implemented at the chain level by combining the canonical identification $C_{\bullet} \simeq k \otimes_{k} C_{\bullet}$ with the cap product with the Connes cyclic cochain complex of $k$ (see [7, 8, 9, ). In fact, the cyclic cohomology of $k$ is a polynomial ring over $k$ generated by the 2-cocycle such that $\sigma(1,1,1)=1$. The action of $\sigma$ on $C_{\bullet}^{\lambda}$ is implemented by the chain map $S: C_{\bullet}^{\lambda} \rightarrow C_{\bullet-2}^{\lambda}$ given by

$$
S\left(x^{\lambda}\right)=\frac{1}{(m-1) m} \sum_{0 \leqslant i<j \leqslant m}(-1)^{i+j}\left(d_{i} d_{j} x\right)^{\lambda}, \quad x \in C_{m}, m \geqslant 2 .
$$

This is Connes' periodicity operator. In particular, this turns the chain complex $C^{\lambda}$ into an $S$ module. In addition, this operator fits into Connes' exact sequence in cyclic homology,

$$
\cdots \rightarrow H_{\bullet}(C) \stackrel{I}{\longrightarrow} H_{\bullet}^{\lambda}(C) \stackrel{S}{\longrightarrow} H_{\bullet-2}^{\lambda}(C) \stackrel{B}{\longrightarrow} H_{\bullet-1}(C) \rightarrow \cdots,
$$

where $H_{\bullet}(C)$ (resp., $H_{\bullet}^{\lambda}(C)$ ) is the Hochschild (resp., cyclic) homology of $C$ (see [7, 8, 9]).

From now on, we assume that $k \supset \mathbb{Q}$, and let $C=\left(C_{\bullet}, d, t\right)$ be a para-precyclic $k$-module. It is not possible to find a chain map $S: C_{\bullet}^{\lambda} \rightarrow C_{\bullet-2}^{\lambda}$ such that $S \pi^{\text {吅 }}=\pi^{\text {吅 }} u^{-2}$ or $S \bar{\pi}^{\text {仲 }}=\bar{\pi}^{\text {如 }} u^{-2}$. Nevertheless, by Proposition 9.5 the chain map $\bar{\pi}^{\text {叫 }}$ has a right-inverse and chain homotopy leftinverse. Namely, the embedding $\bar{\nu}^{\text {仲 }}: C_{\bullet}^{\lambda} \rightarrow C_{T, \bullet}^{\text {仲 }}$ given by (9.11) and (9.18). Therefore, it is natural to seek for a chain map $S: C_{\bullet}^{\lambda} \rightarrow C_{\bullet-2}^{\lambda}$ such that

$$
\bar{\nu}^{\text {吅 }} S=u^{-2} \bar{\nu}^{\text {仲 }} \quad \text { for all } x \in C_{\bullet} \text {. }
$$

As we shall see this equation is satisfied by a unique $k$-linear chain map of degree -2 and will provide us with a solution of $S \pi^{\text {约 }}=\pi^{\text {蛞 }} u^{-2}$ up to homotopy (see Proposition 11.2 below).

Let $S_{0}: C \bullet \rightarrow C_{\bullet-2}$ be the $k$-linear map defined by

$$
S_{0} x=\pi_{0}^{\text {घh }}\left(u^{-2} \nu_{0}^{\text {घh }}(x)\right), \quad x \in C_{38} .
$$


We also introduce the $k$-linear map $\psi_{0}^{\text {叫 }}: C_{\bullet}^{\text {घ }} \rightarrow C_{\bullet-1}$ given by

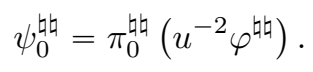

It follows from (9.16) (9.17) that we have

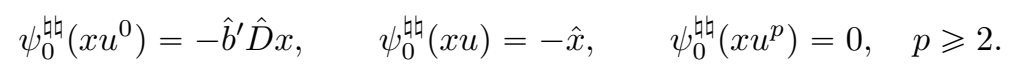

Lemma 11.1. Set $\xi=-\hat{b}^{\prime} \hat{D} b$ and $\eta=-\hat{D} b$. Then

$$
\begin{aligned}
& S_{0}=\xi \hat{N}, \quad S_{0}(1-\tau) x=(1-T) \xi \hat{x}, \quad x \in C_{\bullet},
\end{aligned}
$$

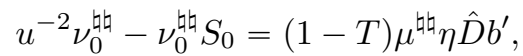

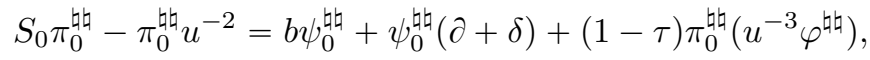

$$
\begin{aligned}
& S_{0} b=b S_{0}+(1-\tau) \eta \xi \hat{N}+(1-T) \xi \hat{b}^{\prime} \hat{D} \text {. }
\end{aligned}
$$

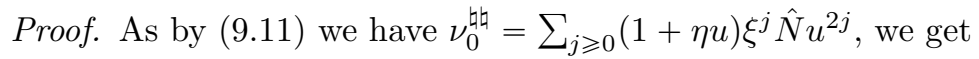

$$
u^{-2} \nu_{0}^{\text {叫 }}=\sum_{j \geqslant 1}(1+\eta u) \xi^{j} \hat{N} u^{2 j-2}=\sum_{j \geqslant 0}(1+\eta u) \xi^{j+1} \hat{N} u^{2 j} .
$$

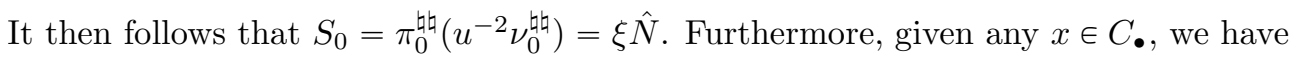

$$
S_{0}(1-\tau) x=\xi N(1-\tau) \hat{x}=\xi(1-T) \hat{x}=(1-T) \xi \hat{x} .
$$

As $S_{0}=\xi \hat{N}$ we have $\nu_{0}^{\text {घh }} S_{0}=\sum_{j \geqslant 0}(1+\eta u) \xi^{j} \hat{N} \xi \hat{N} u^{2 j}$. Combining this with (11.8) and using the equality $\hat{N}+(1-\tau) \hat{D}=1$ gives

$$
\nu_{0}^{\text {吅 }} S_{0}-u^{-2} \nu_{0}^{\text {叫 }}=\sum_{j \geqslant 0}(1+\nu u) \xi^{j}(\hat{N}-1) \xi \hat{N}=-\sum_{j \geqslant 0}(1+\eta u) \xi^{j} \hat{D}(1-\tau) \xi \hat{N} .
$$

As by (9.15) we have $\mu^{\natural \natural}(x)=\sum_{j \geqslant 0}(1+\eta u) \xi^{j} \hat{x}, x \in C_{\bullet}$, we get

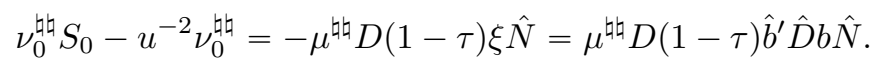

Thanks to the equality $(1-\tau) b=(1-\tau) b^{\prime}$ we have

$$
D(1-\tau) \hat{b}^{\prime} \hat{D} b=\hat{D} b(1-\tau) \hat{D} b=\hat{D} b \hat{D}(1-\tau) b=\hat{D} b \hat{D} b^{\prime}(1-\tau) .
$$

Thus,

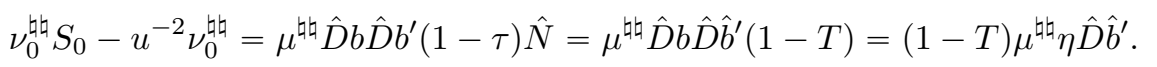

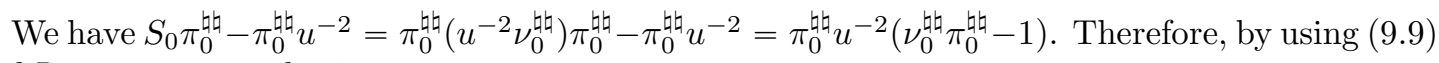
and Lemma 9.1 we obtain

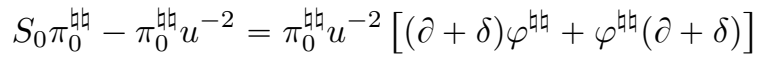

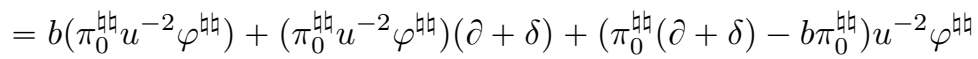

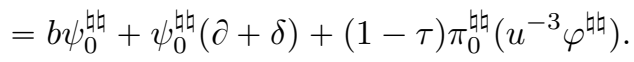

It remains to prove (11.7). By using (9.8) we see that $S_{0} b$ is equal to

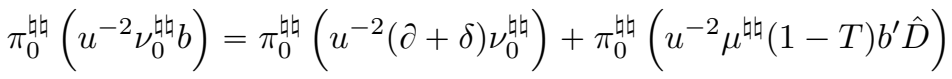

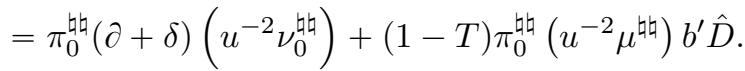

In the same way as in (11.8) we have $u^{-2} \mu^{\text {吼 }}(x)=\sum_{j \geqslant 0}(1+\eta u) \xi^{j+1} \hat{x} u^{2 j}, x \in C_{\bullet}$, and so we see that $\pi_{0}^{\text {吅 }}\left(u^{-2} \mu^{\text {吅 }}(x)\right)=\xi \hat{x}$. Thus,

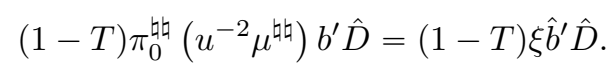

By using (9.8) we also see that $\pi^{\text {虾 }}(\partial+\delta)\left(u^{-2} \nu_{0}^{\text {蛞 }}\right)$ is equal to

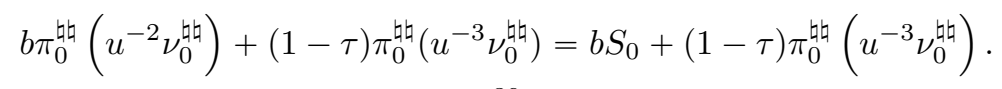




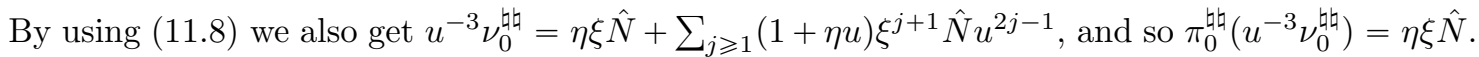
Therefore, we see that $\pi^{\text {旳 }}(\partial+\delta)\left(u^{-2} \nu_{0}^{\text {叫 }}\right)=b S_{0}+\eta \xi \hat{N}$. Combining this with (11.9) and (11.10) gives (11.7). The proof is complete.

As (11.4) implies that $S_{0}$ maps $\operatorname{ran}(1-\tau)$ to $R_{\bullet}^{T} \subset \operatorname{ran}(1-\tau)$, we see that $S_{0}$ descends to a unique $k$-linear map,

$$
S: C_{\bullet}^{\lambda} \longrightarrow C_{\bullet-2}, \quad S \pi^{\lambda}=\pi^{\lambda} S_{0} .
$$

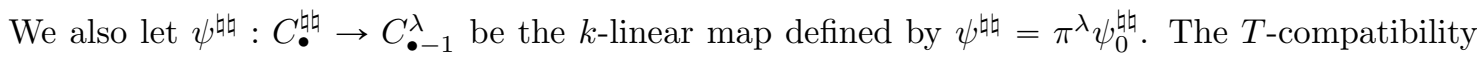

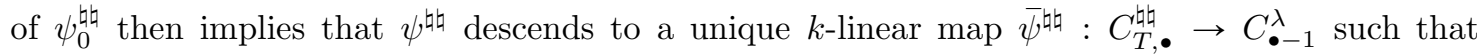

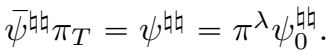

Proposition 11.2. Let $C$ be a para-precyclic $k$-module with $k \supset \mathbb{Q}$.

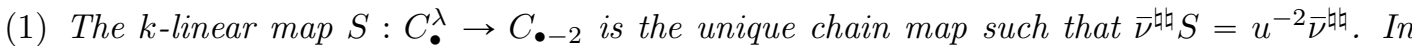
particular, $\left(C^{\lambda}, S\right)$ is an $S$-module and the chain map $\bar{\nu}^{\text {仲 }}: C_{\bullet}^{\lambda} \rightarrow C_{T, \bullet}^{\text {叫 }}$ is an $S$-map.

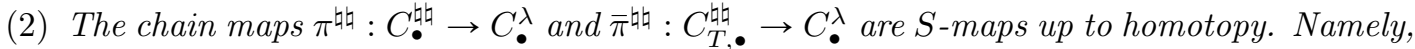

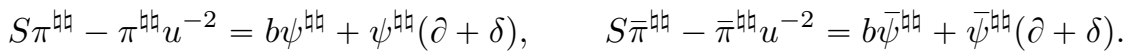

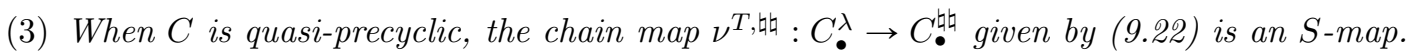

Proof. As $S \pi^{\lambda}=\pi^{\lambda} S_{0}$, we have $(S b-b S) \pi^{\lambda}=S \pi^{\lambda} b-b \pi^{\lambda} S_{0}=\pi^{\lambda}\left(S_{0} b-b S_{0}\right)$. As (11.7) implies that $\operatorname{ran}\left(S_{0} b-b S_{0}\right) \subset \operatorname{ran}(1-\tau)$, we deduce that $(S b-b S) \pi^{\lambda}=0$. This shows that $S b=b S$ on $C_{\bullet}^{\lambda}$, i.e., $S: C_{\bullet}^{\lambda} \rightarrow C_{\bullet-2}$ is a chain map. In particular, the pair $\left(C^{\lambda}, S\right)$ is an $S$-module.

By using the equality $\bar{\nu}^{\text {蚂 }} \pi^{\lambda}=\pi_{T} \nu_{0}^{\text {घh }}$ we also get

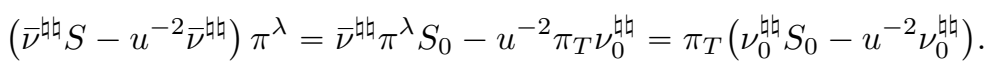

Combining this with (11.5) gives $\left(\bar{\nu}^{\text {吅 }} S-u^{-2} \bar{\nu}^{\natural \natural}\right) \pi^{\lambda}=-\pi_{T}(1-T) \mu^{\natural \natural} \eta \hat{D} b^{\prime}=0$. It then follows

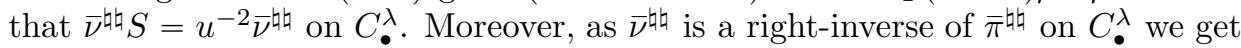

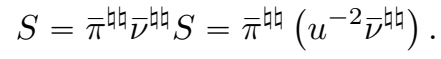

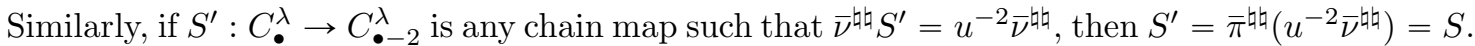

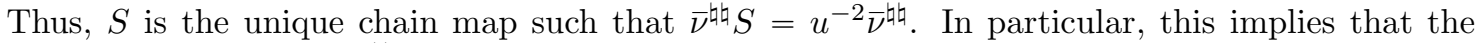
chain map $\bar{\nu}^{\text {狆 }}: C_{\bullet}^{\lambda} \rightarrow C_{T, \bullet}^{\text {犻 }}$ is an $S$-map.

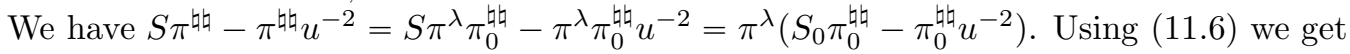

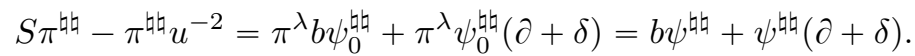

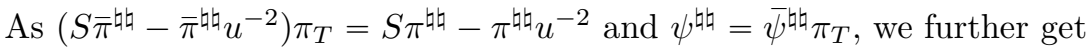

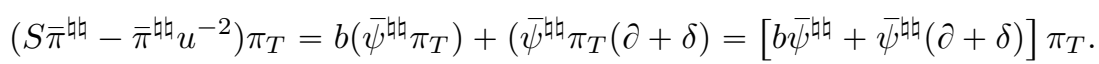

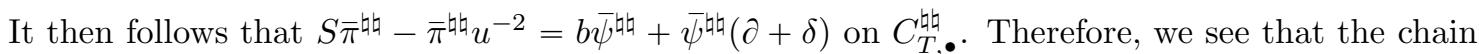
maps $\pi^{\text {如 }}$ and $\pi^{\text {如 }}$ are $S$-maps up to homotopy,

Suppose now that $C$ is a quasi-precyclic $k$-module. By its very definition (9.22) the chain map

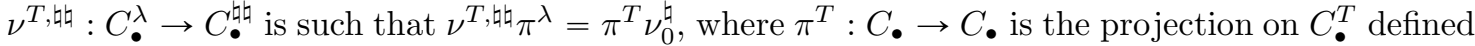
by the splitting $C_{\bullet}=C_{\bullet}^{T} \oplus R_{\bullet}^{T}$. Therefore, as above we have

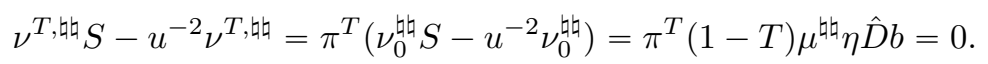

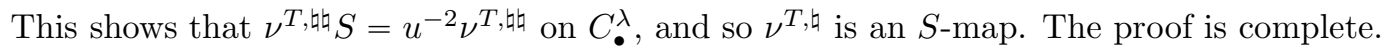

When $C$ is a precyclic $k$-module the chain maps $\bar{\nu}^{\text {घh }}$ and $\nu^{T \text {, 吼 }}$ both agree with the chain map $\nu^{\text {蚛 }}: C_{\bullet}^{\lambda} \rightarrow C_{\bullet}^{\text {叫 }}$ given by (9.20). Therefore, in this case we obtain the statement.

Corollary 11.3 (compare 34). Suppose that $C$ is a precyclic $k$-module with $k \supset \mathbb{C}$.

(1) $S: C_{\bullet}^{\lambda} \rightarrow C_{\bullet-2}$ is the unique chain map such that $\nu^{\text {明 }} S=u^{-2} \nu^{\text {如 }}$.

(2) The chain map $\nu^{\text {如 }}: C_{\bullet}^{\lambda} \rightarrow C_{\bullet}^{\text {切 }}$ is an $S$-map. 
Remark 11.4. We recover the original version of Corollary 11.3 in 34 by replacing $\hat{D}$ by $-\check{D}$ in the formula (9.6) for $\varphi$ (where $\check{D}$ is given by (9.4)), converting $\varphi$ into a special chain homotopy $\check{\varphi}$

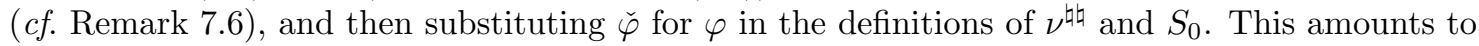
replace $S_{0}$ by the operator,

$$
\check{S}_{0}:=\hat{b}^{\prime}(1-\tau) \check{D}^{2} b \hat{N} \text {. }
$$

In fact, when $C$ is precyclic it can be shown that $S_{0}$ and $\check{S}_{0}$ descend to the same operator on $C_{\bullet}^{\lambda}$ (see Remark 11.7), and so in the precyclic case we recover the $S$-operator of [34].

Let $C=\left(C_{\bullet}, d, s, t\right)$ be an $H$-unital para-precyclic $k$-module. We shall now re-interpret the operator $S$ in terms of the maps $\pi^{\natural}$ and $\nu_{0}^{\natural}$.

In the following, we let $\psi^{\natural}: C_{\bullet}^{\natural} \rightarrow C_{\bullet-1}^{\lambda}$ and $\bar{\psi}^{\natural}: C_{T, \bullet}^{\natural} \rightarrow C_{\bullet-1}$ be the $k$-linear maps defined by $\psi^{\natural}=\psi^{\natural \natural} I$ and $\bar{\psi}^{\natural}=\bar{\psi}^{\natural \natural} I$. In fact, it follows from (11.3) that $\psi^{\natural}=\bar{\psi} \pi_{T}=\pi^{\lambda} \psi_{0}^{\natural}$, where $\psi_{0}^{\natural}: C_{\bullet}^{\natural} \rightarrow C_{\bullet-1}$ is the $k$-linear map given by

$$
\psi_{0}^{\natural}\left(x u^{0}\right)=-b^{\prime} \hat{D} x, \quad \psi_{0}^{\natural}(x u)=-\hat{x}-b^{\prime} \hat{D} s^{\prime} N x, \quad \psi_{0}^{\natural}\left(x u^{p}\right)=0, p \geqslant 2 .
$$

Proposition 11.5. Let $C$ be an $H$-unital para-precyclic $k$-module with $k \supset \mathbb{Q}$.

(1) For all $x \in C_{\bullet}$, we have

(2) The chain map $\bar{\nu}^{\natural}: C_{\bullet}^{\lambda} \rightarrow C_{T, \bullet}^{\natural}$ is an $S$-map.

(3) The chain maps $\pi^{\natural}: C_{\bullet}^{\natural} \rightarrow C_{\bullet}^{\lambda}$ and $\bar{\pi}^{\natural}: C_{T, \bullet}^{\natural} \rightarrow C_{\bullet}^{\lambda}$ are $S$-maps up to homotopy. Namely,

$$
S \pi^{\natural}-\pi^{\natural} u^{-1}=b \psi^{\natural}+\psi^{\natural}\left(b+B u^{-1}\right), \quad S \bar{\pi}^{\natural}-\bar{\pi}^{\natural} u^{-1}=b \bar{\psi}^{\natural}+\bar{\psi}^{\natural}\left(b+B u^{-1}\right) .
$$

(4) When $C$ is quasi-precyclic, the chain map $\nu^{T, \natural}: C_{\bullet}^{\lambda} \rightarrow C_{\bullet}^{\natural}$ given by (10.12) is an $S$-map.

(5) When $C$ is precyclic, the chain map $\nu^{\natural}: C_{\bullet}^{\lambda} \rightarrow C_{\bullet}^{\natural}$ given by (10.11) is an $S$-map.

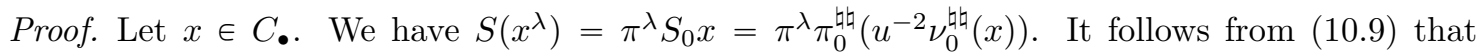

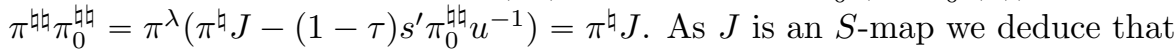

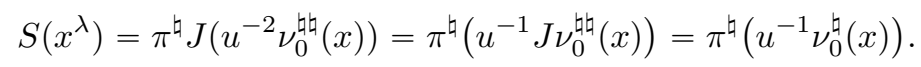

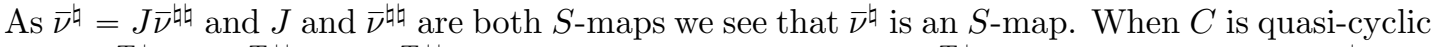

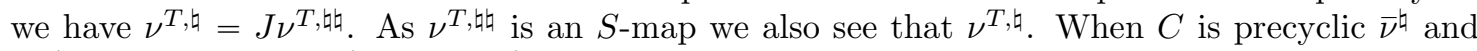
$\nu^{T, \natural}$ both agree with $\nu^{\natural}$, and so $\nu^{\natural}$ is an $S$-map.

We have $S \pi^{\natural}-\pi^{\natural} u^{-1}=S \pi^{\text {吅 }} I-\pi^{\text {吅 }} I u^{-1}=\left(S \pi^{\text {吅 }}-\pi^{\text {吅 }} u^{-2}\right) I$. Thus, by using (11.12) we get

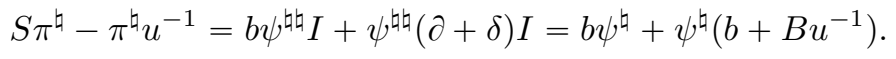

Likewise, we have $S \bar{\pi}^{\natural}-\bar{\pi}^{\natural} u^{-1}=b \bar{\psi}^{\natural}+\bar{\psi}^{\natural}\left(b+B u^{-1}\right)$. The proof is complete.

11.2. Explicit formulas for $S$. We shall now give a simple formula for the operator $S$ and relate it to Connes' original periodicity operator (11.1).

Lemma 11.6. We have $\pi^{\lambda} b^{\prime} \hat{D} b N=\pi^{\lambda} d \hat{D} d N$.

Proof. Set $d^{\prime}=b-b^{\prime}$, i.e., $d^{\prime}=(-1)^{m} d$ on $C_{m}$. We then have

$$
\begin{aligned}
\pi^{\lambda} b^{\prime} \hat{D} b N & =\pi^{\lambda} b \hat{D} b N-\pi^{\lambda} d^{\prime} \hat{D} b N \\
& =\pi^{\lambda} b \hat{D} b N-\pi^{\lambda} d^{\prime} \hat{D} b^{\prime} N-\pi^{\lambda} d^{\prime} \hat{D} d^{\prime} N \\
& =\pi^{\lambda} b \hat{D} b N-\pi^{\lambda} d^{\prime} N \hat{D} b+\pi^{\lambda} d \hat{D} d N
\end{aligned}
$$

where we have used the fact that $\hat{D} b^{\prime} N=\hat{D} N b=N \hat{D} b$.

The equality $b(1-\tau)=(1-\tau) b^{\prime}$ implies that $\pi^{\lambda} b \tau=\pi^{\lambda} b$. More generally, for any polynomial $P(X) \in k[X]$, we have $\pi^{\lambda} b P(\tau)=P(1) \pi^{\lambda} b$. By definition $\hat{D}=m^{-1} D_{m-1}(\tau)$ on $C_{m-1}$, where $D_{m}(X)$ is given by (9.2). Therefore, on $C_{m}$ we have

$$
\pi^{\lambda} b \hat{D} b=m^{-1} \pi^{\lambda} b D_{m-1}(\tau) b=m^{-1} D_{m-1}(1) \pi^{\lambda} b^{2}=0 .
$$


In particular, we see that $\pi^{\lambda} b \hat{D} b N=0$.

The relations (4.4) imply that, on $C_{m}$ and for $j=0, \ldots, m$, we have

$$
d^{\prime} \tau^{j}=(-1)^{m+m j} d t^{j}=(-1)^{m+m j} t^{j} d_{m-j}=(-1)^{m-j} \tau^{j} d_{m-j} .
$$

Thus,

$$
d^{\prime} N=\sum_{0 \leqslant j \leqslant m} d^{\prime} \tau^{j}=\sum_{0 \leqslant j \leqslant m}(-1)^{m-j} \tau^{j} d_{m-j}=\sum_{0 \leqslant j \leqslant m}(-1)^{j} \tau^{m-j} d_{j} .
$$

Therefore, we have $\pi^{\lambda} d^{\prime} N=\sum_{j=0}^{m}(-1)^{j} \pi^{\lambda} \tau^{m-j} d_{j}=\sum_{j=0}^{m}(-1)^{j} \pi^{\lambda} d_{j}=\pi^{\lambda} b$. Using (11.17) we then deduce that $\pi^{\lambda} d^{\prime} \hat{D} N b=\pi^{\lambda} d^{\prime} N \hat{D} b=\pi^{\lambda} b \hat{D} b=0$. Combining this with (11.16) and the equality $\pi^{\lambda} b \hat{D} b N=0$ gives $\pi^{\lambda} b^{\prime} \hat{D} b N=\pi^{\lambda} d \hat{D} d N$. The proof is complete.

Remark 11.7. By using (11.17) and the equalities $\check{D}=-\hat{D}+N$ and $(1-\tau) \check{D}=\hat{N}-1$, it can be shown that, when $C$ is precyclic, $\pi^{\lambda} \check{S}_{0}=\pi^{\lambda} S_{0}$, where $\check{S}_{0}$ is given by (11.13).

We are now in a position to prove the following simple formulas for the operator $S$.

Proposition 11.8. Let $C$ be a para-precyclic $k$-module, and assume that $k \supset \mathbb{Q}$. Then, for all $x \in C_{m}, m \geqslant 2$, we have

$$
\begin{aligned}
S\left(x^{\lambda}\right) & =\frac{-1}{(m-1)}(d \hat{D} d \hat{N} x)^{\lambda} \\
& =\frac{1}{(m-1) m} \sum_{0 \leqslant i<j \leqslant m}(-1)^{i+j}\left(d_{i} d_{j} x\right)^{\lambda} .
\end{aligned}
$$

In particular, we recover Connes' periodicity operator when $C$ is a precyclic $k$-module .

Proof. Let $x \in C_{m}, m \geqslant 2$. By using (11.4) and Lemma 11.6 we obtain

$$
S\left(x^{\lambda}\right)=\pi^{\lambda} S_{0} x=\pi^{\lambda} \xi \hat{N} x=-(m-1)^{-1} \pi^{\lambda} b^{\prime} \hat{D} b \hat{N} x=-(m-1)^{-1}=(d \hat{D} d \hat{N} \hat{x})^{\lambda} .
$$

This gives the first equality in (11.19). Recall that $\hat{D}=m^{-1} D_{m-1}(\tau)$, where $D_{m-1}(X)$ is given by (9.2). Thus,

$$
S\left(x^{\lambda}\right)=\frac{-1}{(m+1) m(m-1)}\left(d D_{m-1}(\tau) d N x\right)^{\lambda} .
$$

It is convenient to introduce the following notation. Given $k$-linear maps $f_{1}: C_{m} \rightarrow C_{m^{\prime}}$ and $f_{2}: C_{m} \rightarrow C_{m^{\prime}}$ we shall write $f_{1} \equiv f_{2}$ when $f_{1}-f_{2}=(1-\tau) g$ for some $k$-linear map $g: C_{m} \rightarrow C_{m^{\prime}}$. In particular, this implies that $\pi^{\lambda} f_{1}=\pi^{\lambda} f_{2}$. Using this notation, we see that in order to prove the 2 nd equality in (11.19) it is enough to show that on $C_{m}$ we have

$$
d D_{m-1}(\tau) d N \equiv-(m+1) \sum_{0 \leqslant i<j \leqslant m}(-1)^{i+j} d_{i} d_{j} .
$$

It follows from (9.2) and (11.18) that on $C_{m}$ the operator $D_{m-1}(\tau) d N$ is equal to

$$
\sum_{\substack{0 \leqslant i \leqslant m-1 \\ 0 \leqslant j \leqslant m}}(-1)^{m+j}(m-1-i) \tau^{i+m-j} d_{j}=\sum_{\substack{0 \leqslant i \leqslant m-1 \\ 0 \leqslant j \leqslant m}}(-1)^{m+j}(m-i-1) T \tau^{i-j} d_{j}
$$

Thus,

$$
d D_{m-1}(\tau) d N=-\sum_{\substack{0 \leq i \leq m-1 \\ 0 \leqslant j \leqslant m}}(-1)^{m-1+j}(m-i-1) T d_{m-1} \tau^{i-j} d_{j} \equiv-\Delta^{(1)}-\Delta^{(2)},
$$

where we have set

$$
\begin{aligned}
\Delta^{(1)} & =\sum_{0 \leqslant i<j \leqslant m}(-1)^{m-1+j}(m-i-1) d_{m-1} \tau^{i-j} d_{j}, \\
\Delta^{(2)} & =\sum_{0 \leqslant j \leqslant i \leqslant m-1}(-1)^{m-1+j}(m-i-1) d_{m-1} \tau^{i-j} d_{j} .
\end{aligned}
$$


If $0 \leqslant i<j \leqslant m$, then on $C_{m-1}$ we have

$$
d_{m-1} \tau^{i-j}=d_{m-1} T^{-1} \tau^{m-(j-i)}=(-1)^{m-(i-j)} T^{-1} \tau^{m-(j-i)} d_{j-i-1} \equiv(-1)^{m-i+j} d_{j-i-1} .
$$

Combining this with the change of index $i \rightarrow j-i-1$ gives

$$
\Delta^{(1)} \equiv \sum_{0 \leqslant i<j \leqslant m}(-1)^{i-1}(m-i-1) d_{j-i-1} d_{j} \equiv \sum_{0 \leqslant i<j \leqslant m-1}(-1)^{i+j}(m+j-i) d_{i} d_{j} .
$$

If $0 \leqslant j \leqslant i \leqslant m-1$, then $d_{m-1} \tau^{i-j}=(-1)^{i-j} \tau^{i-j} d_{m-i+j-1} \equiv(-1)^{i-j} d_{m-i+j-1}$. Thus,

$$
\begin{aligned}
\Delta^{(2)} & \equiv \sum_{0 \leqslant j \leqslant i \leqslant m-1}(-1)^{m+j-1}(m-i-1) d_{m-i+j-1} d_{j} \\
& \equiv \sum_{0 \leqslant j \leqslant i \leqslant m-1}(-1)^{i+j}(i-j) d_{i} d_{j},
\end{aligned}
$$

where we have used the change of index $i \rightarrow m-i+j-1$ to get the 2 nd line. As $d_{i} d_{j}=d_{j} d_{i+1}$ for $i \geqslant j$, we further obtain

$$
\Delta^{(2)} \equiv \sum_{0 \leqslant j \leqslant i \leqslant m-1}(-1)^{i+j}(i-j) d_{j} d_{i+1} \equiv \sum_{0 \leqslant j<i \leqslant m-1}(-1)^{i+j-1}(i-j-1) d_{j} d_{i} .
$$

Upon interchanging the indices $i$ and $j$ we then get

$$
\Delta^{(2)} \equiv \sum_{0 \leqslant j<i \leqslant m-1}(-1)^{i+j-1}(j-i-1) d_{i} d_{j} \equiv \sum_{0 \leqslant j<i \leqslant m-1}(-1)^{i+j}(i-j+1) d_{i} d_{j} .
$$

Combining this with (11.21) and (11.22) gives (11.20). As mentioned above this proves the 2nd equality in (11.19). The proof is complete.

11.3. Applications to periodic cyclic homology. In Connes gave There is a well known formula expressing the periodicity operator $S$ in terms of the $(b, B)$-operators in cyclic cohomology due to Connes [9, Lemma II.34]. As an application of Proposition 11.5 we shall obtain a dual version of Connes' formula for arbitrary $H$-unital para-precyclic $k$-modules.

Let $C=\left(C_{\bullet}, d, s, t\right)$ be an $H$-unital para-precyclic $k$-module. We have

$$
B(1-\tau)=(1-\tau) s^{\prime} N(1-\tau)=(1-\tau) s(1-T)=(1-T)(1-\tau) s^{\prime} .
$$

Therefore, the operator $B$ maps $\operatorname{ran}(1-\tau)$ to $R_{\bullet}^{T}$, and so it descends to a unique $k$-linear map,

$$
B: C_{\bullet}^{\lambda} \longrightarrow C_{T, \bullet}, \quad B \pi^{\lambda}=\pi_{T} B .
$$

Thus, given any $x \in C_{\bullet}$, we have $B\left(x^{\lambda}\right)=\overline{B x}=B \bar{x}$ (where, as above, ${ }^{-}$denotes the class in $C_{T, \bullet}$ ). We then have the following dual version of Connes' formula.

Proposition 11.9. Suppose that $k \supset \mathbb{Q}$. Let $x \in C$ • be such that bx $\operatorname{ran}(1-\tau)$. Then, in $C_{T, \bullet}$ we have

$$
B \circ S\left(x^{\lambda}\right)=-b \bar{x} \quad \bmod \operatorname{ran}[b(1-\tau)] .
$$

Proof. Let $x \in C$. be such that $b x \in \operatorname{ran}(1-\tau)$. Thanks to (11.15) we have

$$
B \circ S\left(x^{\lambda}\right)=B \pi^{\lambda} \pi_{0}^{\natural}\left(u^{-1} \nu_{0}^{\natural}(x)\right)=\pi_{T} B \pi_{0}^{\natural}\left(u^{-1} \nu_{0}^{\natural}(x)\right)=\pi_{T} \pi_{0}^{\natural}\left(B u^{-1} \nu_{0}^{\natural}(x)\right) .
$$

By (10.3) we have $B u^{-1} \nu_{0}^{\natural}(x)=-b \nu_{0}^{\natural}(x)+\nu_{0}^{\natural}(b x) \bmod R_{\bullet}^{T}$. Thus,

$$
B \circ S\left(x^{\lambda}\right)=-\pi_{T} \pi_{0}^{\natural} b \nu_{0}^{\natural}(x)+\pi_{T} \pi_{0}^{\natural} \nu_{0}^{\natural}(b x)=-b \pi_{T}\left[\pi_{0}^{\natural} \nu_{0}^{\natural}(x)\right]+\pi_{T}\left[\pi_{0}^{\natural} \nu_{0}^{\natural}(b x)\right] .
$$

As (10.4) implies that $\pi_{0}^{\natural} \nu_{0}^{\natural}(x)-x \in \operatorname{ran}(1-\tau)$, we see that $b \pi_{T}\left[\pi_{0}^{\natural} \nu_{0}^{\natural}(x)\right]=-b \bar{x} \bmod \operatorname{ran}[b(1-\tau)]$. By (10.8) we also have $\pi_{0}^{\natural} \nu_{0}^{\natural}=\left[1-(1-\tau) s^{\prime} \hat{D} b\right] \hat{N}$. By assumption $b x=(1-\tau) y$ for some $y \in C \bullet-1$. Thus,

$$
\pi_{T}\left[\pi_{0}^{\natural} \nu_{0}^{\natural}(b x)\right]=\pi_{T}\left\{\left[1-(1-\tau) s^{\prime} \hat{D} b\right] \hat{N}(1-\tau) y\right\}=\left[1-(1-\tau) s^{\prime} \hat{D} b\right] \pi_{T}[(1-T) \hat{y}]=0 .
$$

Combining this with (11.23) gives $B \circ S\left(x^{\lambda}\right)=-b \bar{x} \bmod \operatorname{ran} b(1-\tau)$. The proof is complete. 
We still assume that $C$ is an $H$-unital para-precyclic $k$-module. We then can form its periodic para-complex $C^{\sharp}=\left(C_{\bullet}^{\sharp}, b+B, T\right)$, where $C_{\bullet}^{\sharp}$ is defined as in (3.2). When $C$ is precyclic we actually get a chain complex whose homology is denoted by HP. $(C)$. In particular, $C_{T}^{\sharp}$ is a chain complex, and so we can define the periodic cyclic homology HP. $\left(C_{T}\right)$. We have a natural chain $\operatorname{map} \bar{\pi}^{\sharp}: C_{T, i}^{\sharp} \rightarrow \prod_{q \geqslant 0} C_{2 q+i}^{\lambda}$ given by

$$
\bar{\pi}^{\sharp}\left[\left(\bar{x}_{2 q+i}\right)_{q \geqslant 0}\right]=\left(x_{2 q+i}^{\lambda}\right)_{q \geqslant 0}, \quad x_{2 q+i} \in C_{2 q+i} .
$$

Suppose that $k \supset \mathbb{Q}$. As $\left(C^{\lambda}, S\right)$ is an $S$-module, we may also form its periodic chain complex $C^{\lambda, \sharp}:=\left(C_{\bullet}^{\lambda, \sharp}, b\right)$, where $C_{\bullet}^{\lambda, \sharp}$ is defined as in (2.2). We denote by $H_{\bullet}^{\lambda, \sharp}(C)$ its homology. We have an inclusion of chain complexes $C_{\bullet}^{\lambda, \sharp} \subset \prod_{q \geqslant 0} C_{2 q+\bullet}^{\lambda}$. At the homology level this gives rise to a canonical $\left(\mathbb{Z}_{2}\right.$-graded) $k$-linear map $H_{\bullet}^{\lambda, \sharp} \rightarrow \prod_{q \geqslant 0} H_{2 q+\bullet}^{\lambda}(C)$, which fits into the exact sequence (2.3). In particular its range is precisely the inverse limit $\varliminf_{S} H_{2 q+\bullet}^{\lambda}(C)$.

As $k \supset \mathbb{Q}$ we have the chain map $\bar{\nu}^{\natural}: C_{\bullet}^{\lambda} \rightarrow C_{T}^{\natural}$, given by (10.10). By Proposition 10.2 this is a right-inverse and chain homotopy left-inverse of the canonical chain map $\bar{\pi}^{\natural}: C_{T, \bullet}^{\natural} \rightarrow C_{\bullet}^{\lambda}$. By Proposition 11.5 this is an $S$-map, and so it gives rise to a chain map $\bar{\nu}^{\sharp}: C_{\bullet}^{\lambda, \sharp} \rightarrow C_{T, \bullet}^{\sharp}$. This uses the identification $\lim _{u^{-1}} C_{T, \bullet}^{\natural} \simeq C_{T, \bullet}^{\sharp}$ given by $\left(\bar{x}_{2 q+i}\right)_{q \geqslant 0} \rightarrow\left(\pi_{0}^{\natural}\left(\bar{x}_{2 q+i}\right)\right)_{q \geqslant 0}$. As (10.7) implies that $\pi_{0}^{\natural} \nu_{0}^{\natural}=\left[1-(1-\tau) s^{\prime} \hat{D} b\right] \hat{N}$, we have

$$
\bar{\nu}^{\sharp}\left[\left(x_{2 q+i}^{\lambda}\right)_{q \geqslant 0}\right]=\left(\left[1-(1-\tau) s^{\prime} \hat{D} b\right] \hat{N} \bar{x}_{2 q+i}\right)_{q \geqslant 0}, \quad\left(x_{2 q+i}^{\lambda}\right)_{q \geqslant 0} \in C_{i}^{\lambda, \sharp} .
$$

As $\bar{\pi}^{\sharp} \bar{\nu}^{\sharp}=1$ on $C_{\bullet}^{\lambda, \bullet}$ was we actually get the following commutative diagram of chain maps,

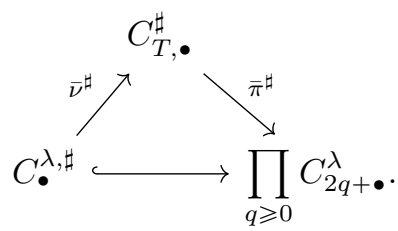

Proposition 11.10. Suppose that $k \supset \mathbb{Q}$.

(1) The chain map $\bar{\nu}^{\sharp}: C_{\bullet}^{\lambda, \sharp} \rightarrow C_{T,}^{\sharp}$ given by (11.25) is a quasi-isomorphism.

(2) The diagram (11.26) gives rise to the following commutative diagram,

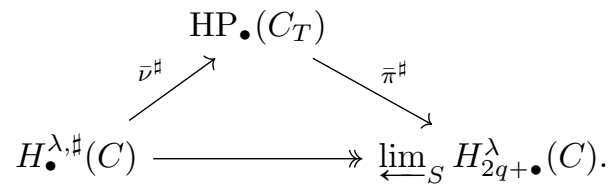

(3) The downward arrow $\bar{\pi}^{\sharp}: \mathrm{HP} \bullet\left(C_{T}\right) \rightarrow \lim _{S} H_{2 q+\bullet}^{\lambda}(C)$ is onto.

Proof. By Proposition 2.2 the very fact that the $S$-map $\bar{\nu}^{\sharp}: C_{\bullet}^{\lambda} \rightarrow C_{T, \bullet}^{\sharp}$ is an $S$-map ensures us that at the periodic level the corresponding chain map $\bar{\nu}^{\sharp}: C_{\bullet}^{\lambda, \sharp} \rightarrow C_{T, \bullet}^{\sharp}$ is a quasi-isomorphism.

At the homology level the commutative diagram (11.26) gives rise to the following commutative diagram,

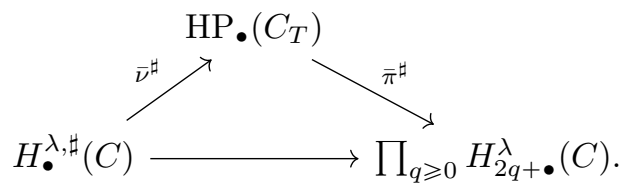

As mentioned above the range of the bottom horizontal arrow is precisely $\lim _{S} H_{2 q+\bullet}^{\lambda}(C)$. Therefore, in order to get the diagram (11.27) it only remains to show that at the homology level $\bar{\pi}^{\sharp}$ maps HP. $\left(C_{T}\right)$ to $\varliminf_{S} H_{2 q+\bullet}^{\lambda} \cdot(C)$. 
Let $\bar{\psi}^{\sharp}: C_{T, \bullet} \rightarrow \prod_{q \geqslant 0} C_{2 q+\bullet}^{\lambda}$ be the $k$-linear map such that, for any $\bar{x}=\left(\bar{x}_{2 q+i}\right)_{q \geqslant 0}$ in $C_{T, i}^{\sharp}$ with $x_{2 q+i} \in C_{2 q+i}$, we have

$$
\psi^{\sharp}(\bar{x})=\left(\psi^{\natural}\left(x_{2 q+i}^{\natural}\right)\right)_{q \geqslant 0},
$$

where the map $\psi^{\natural}: C_{\bullet}^{\natural} \rightarrow C_{\bullet-1}^{\lambda}$ is defined as in Section 11 (see Eq. (11.14)), and we have set $x_{2 q+i}^{\natural}=x_{2 q+i} u^{0}+x_{2 q-2+i} u+\cdots+x_{i} u^{q} \in C_{2 q+i}^{\natural}$.

Claim. On $C_{T, \bullet}^{\sharp}$ we have

$$
S \bar{\pi}^{\sharp}-\bar{\pi}^{\sharp}=b \psi^{\sharp}+\bar{\psi}^{\sharp}(b+B) .
$$

Proof of the Claim. Let $\bar{x}=\left(\bar{x}_{2 q+i}\right)_{q \geqslant 0} \in C_{T, i}^{\sharp}, x_{2 q+i} \in C_{2 q+i}$. We have

$$
\pi^{\sharp}(\bar{x})=\left(x_{2 q+i}^{\lambda}\right)_{q \geqslant 0}=\left(\pi^{\natural}\left(x_{2 q+i}\right)\right)_{q \geqslant 0},
$$

where $x_{2 q+i}^{\natural}$ is defined as above. Note that $u^{-1} x_{2 q+2+i}^{\natural}=x_{2 q+i}^{\natural}$. Thus,

$$
\begin{aligned}
S \bar{\pi}^{\sharp}(\bar{x})-\bar{\pi}^{\sharp}(\bar{x}) & =\left(S \pi^{\natural}\left(x_{2 q+2+i}^{\natural}\right)\right)_{q \geqslant 0}-\left(\pi^{\natural}\left(x_{2 q+i}^{\natural}\right)\right)_{q \geqslant 0} \\
& =\left(\left(S \pi^{\natural}-\pi^{\natural} u^{-1}\right)\left(x_{2 q+2+i}^{\natural}\right)\right)_{q \geqslant 0} .
\end{aligned}
$$

Combining this with Proposition 11.5 we get

$$
S \bar{\pi}^{\sharp}(\bar{x})-\bar{\pi}^{\sharp}(\bar{x})=\left(b \psi^{\natural}\left(x_{2 q+2+i}^{\natural}\right)\right)_{q \geqslant 0}+\left(\psi^{\natural}\left[\left(b+B u^{-1}\right) x_{2 q+2+i}^{\natural}\right]\right)_{q \geqslant 0}
$$

As $b \psi^{\natural}\left(x_{i}^{\natural}\right)=0$, we have $\left(b \psi^{\natural}\left(x_{2 q+2+i}^{\natural}\right)\right)_{q \geqslant 0}=\left(b \psi^{\natural}\left(x_{2 q+i}^{\natural}\right)\right)_{q \geqslant 0}=b \overline{\psi^{\sharp}}(\bar{x})$. Likewise, as $\psi^{\natural}\left(b x_{i}^{\natural}\right)=$ 0 we also have $\left(\psi^{\natural}\left(b x_{2 q+2+i}^{\natural}\right)\right)_{q \geqslant 0}=\bar{\psi}^{\sharp}(b \bar{x})$. In addition, we have

$$
\left(\psi^{\natural}\left(B u^{-1} x_{2 q+2+i}^{\natural}\right)\right)_{q \geqslant 0}=\left(\psi^{\natural}\left(B x_{2 q+i}^{\natural}\right)\right)_{q \geqslant 0}=\bar{\psi}^{\sharp}(B \bar{x}) .
$$

Combining all this with (11.29) then gives

$$
S \bar{\pi}^{\sharp}(\bar{x})-\bar{\pi}^{\sharp}(\bar{x})=b \bar{\psi}^{\sharp}(\bar{x})+\bar{\psi}^{\sharp}[(b+B) \bar{x}] .
$$

This proves the claim.

The above claim implies that $S \bar{\pi}^{\natural}-\bar{\pi}^{\natural}=0$ on HP. $\left(C_{T}\right)$, and so $\bar{\pi}^{\sharp}$ maps HP. $\left(C_{T}\right)$ to $\operatorname{ker}(1-S)=$ $\lim _{S} H_{2 q+\bullet}^{\lambda}(C)$. We thus obtain the commutative diagram (11.27). In that diagram the bottom horizontal arrow is onto and the upward arrow $\bar{\nu}^{\sharp}: H_{\bullet}^{\lambda, \sharp}(C) \rightarrow \mathrm{HP} \bullet\left(C_{T}\right)$ is an isomorphism. It then follows that the downward arrow $\bar{\pi}^{\sharp}: \mathrm{HP} \bullet\left(C_{T}\right) \rightarrow \lim _{S} H_{2 q+\bullet}^{\lambda}(C)$ is onto as well. The proof is complete.

\section{Applications in Cyclic Cohomology}

In this section, we explain the counterparts of Proposition 11.5] and Proposition 11.10 in cyclic cohomology and periodic cyclic cohomology. As we shall see this will provide us with explicit ways to convert $(b, B)$-cocycles into cohomologous periodic cyclic cocycles.

12.1. Para- $S$-comodules. For the sake of exposition's clarity, we give a brief overview of the dual version of para- $S$-modules. We shall call the corresponding objects para-S-comodules. As the dual of a left module is a right module we shall work in the category of right $k$-modules. Thus, a para-S-comodule is given by a system $\left(C^{\bullet}, d, S, T\right)$, where $C^{m}, m \geqslant 0$, are right $k$-modules and $d: C^{\bullet} \rightarrow C^{\bullet+1}$ and $S: C^{\bullet} \rightarrow C^{\bullet+2}$ are $k$-linear maps and $T: C^{\bullet} \rightarrow C^{\bullet}$ is a $k$-linear automorphism satisfying the relations (2.4). In particular, when $T=1$ we get a cochain complex $\left(C^{\bullet}, d\right)$ and $S$ is a true cochain map. In this case we shall denote by $H^{\bullet}(C)$ the corresponding cohomology.

In analogy with para- $S$-modules, we shall call cochain map any map between para- $S$-comodules that is compatible with the $d$-operators. A cochain $S$-map is a cochain map which is compatible with the $(S, T)$-operators. We also have natural notions of cochain homotopies of cochain maps and cochain $S$-homotopies of cochain $S$-maps. This provides us with notions of cochain homotopy equivalence and cochain $S$-homotopy equivalence between para- $S$-comodules, as well as the corresponding notions of deformation retracts and $S$-deformation retract of para- $S$-comodules. 
Our main example of a para-S-module is the dual of a para- $S$-module. Given any left $k$ module $\mathscr{E}$ we denote by $\mathscr{E}^{\dagger}$ the dual right $k$-module $\operatorname{Hom}_{k}(\mathscr{E}, k)$. By duality any $k$-linear map $f: \mathscr{E}_{1} \rightarrow \mathscr{E}_{2}$ gives rise to a $k$-linear map $f^{\dagger}: \mathscr{E}_{2}^{\dagger} \rightarrow \mathscr{E}_{1}^{\dagger}$ such that $f^{\dagger}(\phi)=\phi \circ f \forall \phi \in \mathscr{E}_{2}^{\dagger}$. Any para- $S$-module $C=\left(C_{\bullet}, d, S, T\right)$ then gives rise to a dual para- $S$-comodule $\left(C^{\bullet}, d, S, T\right)$, where $C^{m}=C_{m}^{\dagger}$ and we denote by $(d, S, T)$ the dual maps $\left(d^{\dagger}, S^{\dagger}, T^{\dagger}\right)$. Any chain map (resp., $S$-map) between para- $S$-modules gives rise to a cochain map (resp., cochain $S$-map) between the corresponding dual para- $S$-comodules. Moreover, any chain homotopy equivalence (resp., $S$ homotopy equivalence) of para- $S$-modules gives rise to a cochain homotopy equivalence (resp., cochain $S$-homotopy equivalence) of the dual para- $S$-comodules.

Let $\left(C^{\bullet}, d, S, T\right)$ be a para- $S$-comodule. The operator $S: C^{\bullet} \rightarrow C^{\bullet+2}$ gives rise to directed systems of right $k$-modules $\left\{C^{2 q+m}\right\}_{q \geqslant 0}, m \geqslant 0$, which are compatible with the operators $d$ and $T$. For each $m$, let $C_{\sharp}^{m}=\underline{\lim }_{S} C^{2 q+i}$ be the corresponding direct limit, i.e.,

$$
C_{\sharp}^{m}=\left(\bigoplus_{q \geqslant 0} C^{2 q+i}\right) / \operatorname{ran}(1-S) .
$$

We have a natural identification $C_{\sharp}^{m+2} \simeq C_{\sharp}^{m}$. Under this identification, the operators $d$ and $T$ give rise to operators on $C_{\sharp}^{0} \oplus C_{\sharp}^{1}$ which are odd and even, respectively. Moreover, we have $d^{2}=(1-T)$ on $C_{\sharp}^{0} \oplus C_{\sharp}^{1}$. We thus obtain a $\mathbb{Z}_{2}$-graded cochain paracomplex $C^{\sharp}:=\left(C_{\sharp}^{0} \oplus C_{\sharp}^{1}, d, T\right)$, which we shall call the periodic cochain paracomplex of $C$.

Any cochain $S$-map between para- $S$-comodules gives rise to a $T$-compatible cochain map between the corresponding periodic cochain paracomplexes. Furthermore, any cochain $S$-homotopy equivalence of para- $S$-comodules gives rise to a $T$-compatible cochain homotopy equivalence of the corresponding periodic cochain paracomplexes.

If $C=(C \bullet, d, S, T)$ is a para- $S$-module, then we denote by $C_{\sharp}$ the periodic cochain paracomplex of its dual para- $S$-comodule. There is a natural duality between $C_{\sharp}$ and the periodic chain complex $C^{\sharp}$. Thus, any $S$-map (resp., $S$-homotopy equivalence) between para- $S$-comodules gives rise to a $T$-compatible cochain map (resp., cochain $S$-homotopy equivalence) between the corresponding periodic cochain paracomplexes.

When $T=1$ we obtain a periodic cochain complex $\left(C_{\sharp}^{\bullet}, d\right)$. We denote by $H_{\sharp}^{\bullet}(C)$ its cohomology. As the operator $S: C^{\bullet} \rightarrow C^{\bullet+2}$ is a cochain map, it descends to a $k$-linear map $S: H_{\sharp}^{\bullet}(C) \rightarrow$ $H_{\sharp}^{\bullet+2}(C)$, and so it defines a directed system of cohomology modules. The canonical projection $\oplus_{q \geqslant 0} C^{2 q+\bullet} \rightarrow C_{\sharp}^{\bullet}$ is an $S$-invariant cochain map, and so it descends to a $k$-linear map from $\lim _{S} H^{2 q+\bullet}(C) \rightarrow H_{\sharp}^{\bullet}(C)$. As the direct limit functor is exact (see, e.g., [56]), we actually get a canonical isomorphism,

$$
\lim _{S} H^{2 q+\bullet}(C) \simeq H_{\sharp}^{\bullet}(C) .
$$

12.2. Cyclic cohomology. Let $C=\left(C_{\bullet}, d, s, t\right)$ be an $H$-unital para-precyclic $k$-module. By duality the $H$-unital precyclic $k$-module $C_{T}=\left(C_{T, \bullet}, d, s, t\right)$ gives rise to an $H$-unital precyclic $k$-comodule $\left(C_{T}^{\bullet}, d, s, t\right)$, where

$$
C_{T}^{m}:=\left\{\phi \in \operatorname{Hom}_{k}\left(C_{m}, k\right) ; \phi \circ T=\phi\right\}, \quad m \geqslant 0 .
$$

Here the structural operators $(d, s, t)$ on $C_{T}^{\bullet}$ are the dual versions of the structural operators $(d, s, t)$ on $C_{T, \bullet}$. We let $C_{T, \sharp}:=\left(C_{T, \sharp}^{\bullet}, b+B\right)$ be the corresponding cyclic cochain complex, where

$$
C_{T, \natural}^{m}=C_{T}^{m} \oplus C_{m-2}^{T} \oplus \cdots, \quad m \geqslant 0 .
$$

We denote by $\mathrm{HC}^{\bullet}\left(C_{T}\right)$ the cohomology of $C_{T, \sharp}$. The natural duality between the chain complex $C_{T}^{\natural}$ and the cochain complex $C_{T, \natural}$ is given by

$$
\left\langle\phi, \bar{x} u^{p}\right\rangle:=\left\langle\phi_{m-2 p}, x\right\rangle, \quad x \in C_{m-2 p}, \quad \phi=\left(\phi_{m}, \phi_{m-2}, \ldots\right) \in C_{T, \text { घ }}^{m} .
$$

We also let $C_{\lambda}=\left(C_{\lambda}^{\bullet}, b\right)$ be the Connes cochain complex of $C$, where

$$
C_{\lambda}^{m}:=\left\{\phi \in \operatorname{Hom}_{k}\left(C_{m}, k\right) ; \phi \circ \tau=\phi\right\}, \quad m \geqslant 0 .
$$


We denote by $H_{\lambda}(C)$ the cohomology of $C_{\lambda}$. As the $B$-operator on cochains is annihilated by cyclic cochains, we have an inclusion of cochain complexes,

$$
\bar{\iota}_{\natural}: C_{\lambda}^{\bullet} \longrightarrow C_{T, \natural}^{\bullet}, \quad \bar{\iota}_{\natural}(\phi)=(\phi, 0, \ldots), \quad \phi \in C_{\lambda}^{m} .
$$

This is the dual version of the canonical chain map $\bar{\pi}^{\natural}: C_{T \cdot \bullet}^{\natural} \rightarrow C_{\bullet}^{\lambda}$.

Suppose that $k \supset \mathbb{Q}$. By duality the chain map $\bar{\nu}^{\natural}: C_{\bullet}^{\lambda} \rightarrow C_{T, \bullet}^{\natural}$ gives rise to a cochain map $\bar{\nu}_{\natural}: C_{T, \natural}^{\bullet} \rightarrow C_{\lambda}^{\bullet}$. In fact, in view of (10.10), given any $\phi=\left(\phi_{m}, \phi_{m-2}, \ldots\right) \in C_{T, \natural}^{m}, m \geqslant 0$, we have

$$
\bar{\nu}_{\natural}(\phi)=\sum_{m-2 j \geqslant 0}(-1)^{j} \phi_{m-2 j} \circ\left\{\left[1-(1-\tau) s^{\prime} \hat{D} b\right]\left(\hat{b}^{\prime} \hat{D} b\right)^{j} \hat{N}\right\} .
$$

By Proposition 10.2 the chain map $\bar{\nu}^{\natural}$ is a right-inverse and chain homotopy left-inverse of the canonical chain map $\bar{\pi}^{\natural}$. Therefore, by duality we obtain the following result.

Proposition 12.1. Assume that $k \supset \mathbb{Q}$. Then the cochain map $\bar{\nu}_{\sharp}$ given by (12.6) is a left-inverse and cochain homotopy right-inverse of the canonical inclusion (12.5).

Remark 12.2. When $C$ is a cyclic module the fact that the canonical inclusion (12.5) is a quasiisomorphism goes back to Connes [8, 9].

The upshot of Proposition 12.1 is an explicit way to convert any $(b, B)$-cocycle into a cohomologous periodic cocycle. Namely, we have the following statement.

Corollary 12.3. Assume that $k \supset \mathbb{Q}$. Then, for every cocycle $\phi=\left(\phi_{m}, \phi_{m-2}, \ldots\right) \in C_{T, \mathfrak{\natural}}^{m}, m \geqslant 0$, the cyclic cochain $\bar{\nu}_{\sharp}(\phi)$ given by (12.6) is a cocycle in the same cohomology class as $\phi$.

12.3. Periodic cyclic cohomology. Let $C=\left(C_{\bullet}, d, s, t\right)$ be an $H$-unital para-precyclic $k$-module. Under (12.4) we have a natural duality between the chain complex $C_{T}^{\natural}=\left(C_{T, \bullet}^{\natural}, b+B u^{-1}\right)$ and the cochain complex $C_{T, \natural}=\left(C_{T, \natural}^{\bullet}, b+B\right)$. Under this duality the dual of the projection $u^{-1}: C_{T, \bullet}^{\natural} \rightarrow C_{T, \bullet-2}^{\natural}$ is simply the inclusion of $C_{T, \natural}^{\bullet}=C_{T}^{\bullet} \oplus C_{T}^{\bullet-2} \oplus \cdots$ into $C_{T, \natural}^{\bullet+2}=C_{T}^{\bullet+2} \oplus C_{T}^{\bullet} \oplus \cdots$. This turns $C_{T, \text { }}$ into an $S$-comodule. Its periodic cochain complex is naturally identified with the $\mathbb{Z}_{2}$-graded cochain complex $C_{T, \sharp}=\left(C_{T, \sharp}^{\bullet}, b+B\right)$, where

$$
C_{T, \sharp}^{i}=\bigoplus_{q \geqslant 0} C_{T}^{2 q+i}, \quad i=0,1 .
$$

More precisely, the identification between $C_{T, \sharp}^{i}$ and the direct limit $\lim _{S} C_{T}^{2 q+i}$ is induced from

$$
\left(\phi_{i}, \phi_{2+i}, \ldots, \phi_{2 q_{0}+i}, 0, \ldots\right) \longrightarrow\left(\phi_{i}^{\natural}, \phi_{2+i}^{\natural}, \ldots, \phi_{2 q_{0}+i}^{\natural}, 0, \ldots\right),
$$

where we have set $\phi_{2 q+i}^{\natural}:=\left(\phi_{2 q+i}, \phi_{2 q-2+i}, \ldots\right)$. This identification is the dual version of the identification of $\lim _{u^{-1}} C_{2 q+i}$ with $C_{i}^{\sharp}$ described in Section 3. We denote by $\operatorname{HP}^{\bullet}\left(C_{T}\right)$ the cohomology of the cochain complex $C_{T, \sharp}$. This is the cyclic cohomology of the $H$-unital precyclic module $C_{T}$.

Suppose that $k \supset \mathbb{Q}$. By duality the chain map $S: C_{\bullet}^{\lambda} \rightarrow C_{\bullet_{-2}^{\lambda}}^{\lambda}$ gives rise to a degree 2 cochain map $S: C_{\lambda}^{\bullet} \rightarrow C_{\lambda}^{\bullet+2}$, so that we obtain an $S$-comodule $\left(C_{\bullet}^{\lambda}, b, S\right)$ which is the dual $S$-comodule of the $S$-module $\left(C_{\bullet}^{\lambda}, b, S\right)$. Let $C_{\lambda, \sharp}:=\left(C_{\lambda, \sharp}^{\bullet_{\lambda}}, b, S\right)$ be its periodic cochain complex, where $C_{\lambda, \sharp}^{i}$, $i=0,1$, is defined as in (12.1). We denote by $H_{\lambda, \sharp}(C)$ its cohomology. There is a natural duality between $C_{\lambda, \sharp}$ and the periodic chain complex $C^{\lambda, \sharp}$ introduced in Section 11 .

By duality the chain map $\bar{\pi}^{\sharp}: C_{T, \bullet}^{\sharp} \rightarrow \prod_{q \geqslant 0} C_{2 q+}^{\lambda}$ in (11.24) corresponds to the natural cochain inclusion $\overline{\iota_{\sharp}}: \bigoplus_{q \geqslant 0} C_{\lambda}^{2 q+\bullet} \hookrightarrow C_{T, \sharp}^{\bullet}$. At the cohomology level this gives rise to a ( $\mathbb{Z}_{2}$-graded) $k$ linear map $\overline{\iota_{\sharp}}: \bigoplus_{q \geqslant 0} H_{\lambda}^{2 q+\bullet}(C) \rightarrow \mathrm{HP}^{\bullet}\left(C_{T}\right)$. Although at the level of cochains the inclusion $\overline{\iota_{\sharp}}$ is not $S$-invariant, at the cohomology level we get an $S$-invariant map. This can be seen by using the dual version of Proposition 11.9 ( $c f$. 9]). Alternatively, we know from (11.28) that the chain map $(1-S) \bar{\pi}^{\sharp}$ is chain homotopic to 0 . By duality $\bar{\iota}_{\sharp}(1-S)$ is cochain homotopic to 0 , and hence $\bar{\iota}_{\sharp}(1-S)=0$ on $\bigoplus_{q \geqslant 0} H_{\lambda}^{2 q+\bullet}(C)$. Anyway, we see that at the cohomology level the map $\bar{\iota}_{\sharp}$ descends to a $k$-linear map,

$$
\bar{\iota}^{\sharp}: \lim _{S} H_{2 q+\bullet}^{\lambda} \longrightarrow \mathrm{HP}^{\bullet}\left(C_{T}\right) .
$$


This map was shown by Connes [8, 9] (at least when $k=\mathbb{C}$ and $C$ is the cyclic space of a unital C-algebra).

We shall now use Proposition 11.10 and Proposition 12.1 to reinterpret Connes' isomorphism theorem and exhibit an explicit inverse of $\iota^{\sharp}$ for $H$-unital precyclic modules.

Let $C=(C, d, s, t)$ be an $H$-unital precyclic $k$-module with $k \supset \mathbb{Q}$. It follows from Proposition 11.5 that the cochain map $\bar{\nu}_{\natural}: C_{T, \natural}^{\bullet} \rightarrow C_{\lambda}^{\bullet}$ is a cochain $S$-map. At the periodic level the corresponding cochain map $\bar{\nu}_{\sharp}: C_{T, \sharp}^{\bullet} \rightarrow C_{\lambda, \sharp}^{\bullet}$ is the dual version of the chain map $\bar{\nu}^{\sharp}: C_{\bullet}^{\lambda, \sharp} \rightarrow C_{T, \bullet}^{\sharp}$. Thus, given any periodic cochain $\phi=\left(\phi_{i}, \phi_{2+i}, \ldots\right) \in C_{T, \sharp}^{i}$, we have

$$
\bar{\nu}_{\sharp}(\phi)=\text { class of }\left(\phi_{2 q+i} \circ\left\{\left[1-(1-\tau) s^{\prime} \hat{D} b\right] \hat{N}\right\}\right)_{q \geqslant 0} \bmod \operatorname{ran}(1-S) .
$$

By duality the commutative diagram of chain maps (11.26) then gives rise to the following commutative diagram of cochain maps,

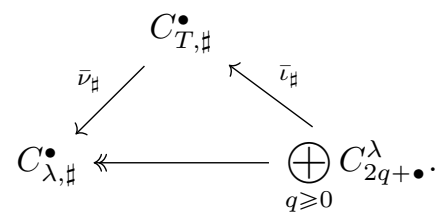

Proposition 12.4. Let $C=(C, d, s, t)$ be an $H$-unital precyclic $k$-module with $k \supset \mathbb{Q}$.

(1) The cochain map $\bar{\nu}_{\sharp}: C_{T, \sharp}^{\bullet} \rightarrow C_{\lambda, \sharp}$ given by (12.8) is a quasi-isomorphism.

(2) The following diagram is commutative,

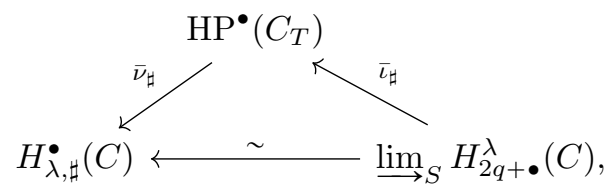

where the bottom horizontal arrow is the canonical isomorphism (12.2).

(3) The Connes map $\bar{\iota}_{\sharp}: \lim _{\longrightarrow} H_{2 q+\bullet}^{\lambda} \rightarrow \mathrm{HP}^{\bullet}\left(C_{T}\right)$ is an isomorphism.

In other words, the cochain map $\bar{\nu}_{\sharp}$ allows us to invert Connes' map $\bar{\iota}_{\sharp}: \varliminf_{\lim _{S}} H_{2 q+}^{\lambda} \rightarrow \operatorname{HP}^{\bullet}\left(C_{T}\right)$ under the canonical identification $\lim _{\longrightarrow} H_{2 q+\bullet}^{\lambda}(C) \simeq H_{\lambda, \sharp}^{\bullet}(C)$. In particular, this allows us to realize Connes' isomorphism by means of an explicit quasi-isomorphism.

Proof. As the cochain map $\bar{\nu}_{\sharp}: C_{T, \sharp}^{\bullet} \rightarrow C_{\lambda, \sharp}^{\bullet}$ arises from the cochain $S$-map $\bar{\nu}_{\sharp}: C_{T, \natural}^{\bullet} \rightarrow C_{\lambda}^{\bullet}$, we have a commutative diagram,

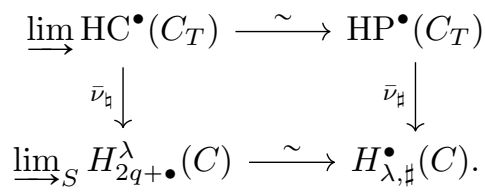

Here the bottom horizontal arrow is the canonical isomorphism (12.2) and the top horizontal arrow arises from this isomorphism under the identification of $C_{T, \sharp}^{\bullet}$ and $\lim _{\longrightarrow, \text { }} C_{\boldsymbol{\bullet}_{\text {, }}}$ given by (12.7). We know by Proposition 12.1 that $\bar{\nu}_{\mathfrak{\natural}}: \mathrm{HC}^{\bullet}\left(C_{T}\right) \rightarrow H_{\lambda}^{\bullet}(C)$ is an isomorphism. This isomorphism is compatible with the periodicity operators in cohomology. Therefore, by functoriality of the direct limit, the left vertical arrow $\bar{\nu}_{\sharp}: \lim _{\longrightarrow} \mathrm{HC}^{\bullet}\left(C_{T}\right) \rightarrow \lim _{S} H_{2 q+\bullet}^{\lambda}(C)$ is an isomorphism. As the horizontal arrows are both isomorphisms, it then follows that the right vertical arrow is an isomorphism as well. That is, the cochain map $\bar{\nu}_{\sharp}: C_{T, \sharp}^{\bullet} \rightarrow C_{\lambda, \sharp}^{\bullet}$ is a quasi-isomorphism. 
At the cohomology level the commutative diagram of cochain maps (12.9) gives rise to the following commutative diagram,

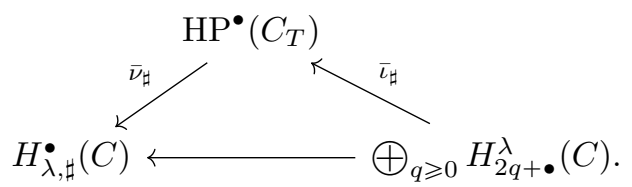

As the bottom horizontal arrow and the map $\bar{\iota}_{\sharp}$ both descend to maps on $\underline{\lim }_{S} H_{2 q+\bullet}^{\lambda}(C)$, we obtain the commutative diagram (12.10). In the diagram (12.10) the bottom vertical arrow is an isomorphism. As shown above, the downward arrow $\bar{\nu}_{\sharp}: \operatorname{HP}^{\bullet}\left(C_{T}\right) \rightarrow H_{\lambda, \sharp}(C)$ is an isomorphism. It then follows that the upward arrow $\bar{\iota}_{\sharp}: \varliminf_{\longrightarrow} H_{2 q+\bullet}^{\lambda}(C) \rightarrow \mathrm{HP}^{\bullet}\left(C_{T}\right)$ is an isomorphism. The proof is complete.

Remark 12.5. Cyclic cohomology is the natural receptacle of the Chern character in $K$-homology, a.k.a. Connes-Chern of character [9]. The original definition of the Connes-Chern character by Connes [9] is in terms of cyclic cocycles. However, we often get better information from its representations by $(b, B)$-cocycles [10, 11, 12. It would be interesting to see what light on these representations could be shed from the convertion results mentioned above.

\section{REFERENCES}

[1] Bauval, A.: Le Théorème d'Eilenberg-Zilber en homologie cyclique entière. Preprint, 1998, arXiv:1611.08437.

[2] Berglund, A.: Homological perturbation theory for algebras over operads. Algebr. Geom. Topol. 14 (2014), 2511-2548.

[3] Brown, R.: The twisted Eilenberg-Zilber theorem, Simposio Di Topologia (Messina, 1964), Edizioni Oderisi, Gubbio, 1965, pp. 33-37.

[4] Bökstedt, M.; Hsiang, W. C.; Madsen, I.: The cyclotomic trace and algebraic K-theory of spaces. Invent. Math. 111 (1993), 465-539.

[5] Burghelea, D.: The cyclic homology of the group rings. Comment. Math. Helv. 60 (1985), 354-365.

[6] Burghelea, D.: Cyclic homology and the algebraic K-theory of k-modules. I. Contemp. Math., 55, Amer. Math. Soc. Providence, RI, 1986, pp. 89-115.

[7] Connes, A.: Spectral sequence and homology of currents for operator algebras. Math. Forschungsinstitut Oberwolfach Tagungsbericht 42/81, Funktionalanalysis und C*-Algebren, 27.9.-3.10, 1981.

[8] Connes, A.: Cohomologie cyclique et foncteur $\mathrm{Ext}^{n}$. C. R. Acad. Sci. Paris Ser. I Math. 296 (1983), 953-958.

[9] Connes, A.: Noncommutative differential geometry. Inst. Hautes Études Sci. Publ. Math. 62 (1985), 257-360.

[10] Connes, A.; Moscovici, H.: Transgression and the Chern character of finite-dimensional K-cycles. Comm. Math. Phys. 155 (1993), 103-122.

[11] Connes, A.; Moscovici, H.: The local index formula in noncommutative geometry. Geom. Funct. Anal. 5 (1995), $174-243$.

[12] Connes, A.; Moscovici, H.: Hopf algebras, cyclic cohomology and the transverse index theorem. Comm. Math. Phys. 198 (1998), 199-246.

[13] Crainic, M.: Cyclic cohomology of étale Groupoids: the general case. K-Theory 17 (1999), 319-362.

[14] Crainic, M.: Cyclic cohomology of Hopf algebras. J. Pure Appl. Algebra 166 (2002), 29-66.

[15] Cuntz, J.; Quillen, D.: Cyclic homology and nonsingularity. J. Amer. Math. Soc. 8 (1995), 373-442.

[16] Dwyer, W.G.; Kan, D.M.: Normalizing the cyclic modules of Connes. Comment. Math. Helv. 60 (1985), $582-600$

[17] Eilenberg, S.; MacLane, S.: Cohomology theory in abstract groups. I. Ann. of Math. 48 (1947), 51-78.

[18] Fiedorowicz, Z.; Loday, J.L.: Crossed simplicial groups and their associated homology. Trans. Amer. Math. Soc. 326 (1991), 57-87.

[19] Feigin, B.P.; Tsygan, B.L.: Additive K-theory. K-theory, arithmetic and geometry (Moscow, 1984-1986), 67-209, Lecture Notes in Math., 1289, Springer, 1987.

[20] Feigin, B.P.; Tsygan, B.L.: Cyclic homology of algebras with quadratic relations, universal enveloping algebras and group algebras. K-theory, arithmetic and geometry (Moscow, 1984-1986), 210-239, Lecture Notes in Math., 1289, Springer, 1987.

[21] Getzler, E.; Jones, J.D.: The cyclic cohomology of crossed product algebras. J. Reine Angew. Math. 445 (1993), $161-174$

[22] Gugenheim, V.K.A.M.: On a the chain complex of a fibration. Illinois J. Math. 3 (1972), 398-414.

[23] Gugenheim, V.K.A.M.; Lambe, L.A.: Perturbation theory in differential homological algebra. I. Illinois J. Math. 33 (1989), 566-582.

[24] Gugenheim, V.K.A.M.; Lambe, L.A.; Stasheff, J.D.: Perturbation theory in differential homological algebra. II. Illinois J. Math. 35 (1991), 357-373. 
[25] Hadfield, T.; Krähmer, U.: Braided homology for quantum groups. J. K-Theory 4 (2009), 299-332.

[26] Hajac, P.M.; Khalkhali, M.; Rangipour, B.; Sommerhäuser, Y.: Hopf-cyclic homology and cohomology with coefficients. C. R. Math. Acad. Sci. Paris 338 (2004), 667-672.

[27] Hood, C.E; Jones, J.D.S.: Some algebraic properties of cyclic homology groups. K-theory 1 (1987), 361-384

[28] Huebschmann, J.; Kadeishvili, T.: Small models for chain algebras. Math. Z. 207 (1991) 245-280.

[29] Hadfield, T.; Krähmer, U.: Twisted homology of quantum SL(2). K-Theory 34 (2005), 327-360.

[30] Hadfield, T.; Krähmer, U.: Twisted homology of quantum SL(2) - Part II. I J. K-Theory 6 (2010), 69-98.

[31] Ji, R.: Nilpotency of Connes' periodicity operator and the idempotent conjectures. K-Theory 9 (1995), 59-76.

[32] Jones, J.D.S.; Kassel, C.: Bivariant cyclic theory. K-Theory 3 (1989) 339-365.

[33] Kassel, C.: Cyclic homology, comodules and mixed complexes. J. Algebra 107 (1987) 195-216.

[34] Kassel, C.: Homologie cyclique, caractère de Chern et lemme de perturbation. J. reine angew. Math. 408 (1990), 159-180.

[35] Kaygun, A.: Bialgebra cyclic homology with coefficients. K-Theory, 34 (2005), 151-194.

[36] Khalkhali, M.; Rangipour, B.: On the generalized cyclic Eilenberg-Zilber Theorem. Canad. Math. Bull. Vol. 47 (1), (2004) 38-48.

[37] Kowalzig, N.; Krähmer, U.: Batalin-Vilkovisky structures on Ext and Tor. J. Reine Angew. Math. 697 (2014), $159-219$.

[38] Krähmer, U.; Madden, D.: Cyclic vs mixed homology. Homology, Homotopy and Applications 20 (2018), 237-250.

[39] Kustermans, J., Murphy, G. J., Tuset, L.: Differential calculi over quantum groups and twisted cyclic cocycles. J. Geom. Phys. 44 (2003), 570-594.

[40] Lambe, L.; Stasheff, J.: Applications of perturbation theory to iterated fibrations. J. Manuscripta Math. 58 (1987), 363-376.

[41] Lapin, S. V.: Differential perturbations and Do-differential modules. Sb. Math. 192 (2001), 1639-1659.

[42] Loday, J.-L.: Cyclic homology. Springer, Berlin, 1992.

[43] Loday, J.-L.; Quillen, D.: Cyclic homology and the Lie algebra homology of matrices. Comment. Math. Helv. 59 (1984), 565-591.

[44] Marciniak, Z.: Cyclic homology of group rings. Geometric and algebraic topology, 305-312, Banach Center Publ., 18, PWN, Warsaw, 1986.

[45] Nistor, V.: Group cohomology and the cyclic cohomology of crossed products. Invent. Math. 99 (1990), 411-424.

[46] Nistor, V.: Cyclic cohomology of crossed products by algebraic groups. Invent. Math. 112 (1993), 615-638.

[47] Nistor, V.: A bivariant Chern-Connes character. Ann. Math. 138 (1993), 555-590.

[48] Ponge, R.: The cyclic homology of crossed-product algebras, I. C. R. Acad. Sci. Paris, sér. I, 355 (2017), 618-622.

[49] Ponge, R.: The cyclic homology of crossed-product algebras, II. C. R. Acad. Sci. Paris, sér. I, 355 (2017), 623-627.

[50] Ponge, R.: Cyclic homology and group actions. J. Geom. Phys. 123 (2018), 30-52.

[51] Ponge, R.: Eilenberg-Zilber theorem and cup product for biparacylic modules. In preparation.

[52] Real, P.: Homological perturbation theory and associativity. Homology Homotopy Appl. 2 (2000), 51-88.

[53] Shih, W.: Homologie des espaces fibrés. Inst. Hautes Études Sci. Publ. Math. 13 (1962), 93-176.

[54] Tsygan, B.L.: Homology of matrix Lie algebras over rings and Hochschild homology. Uspekhi Math. Nawk. 38 (1983), 217-218.

[55] Voigt, C.: Equivariant periodic cyclic homology. J. Inst. Math. Jussieu 6 (2007), 689-763.

[56] Weibel, C.: An Introduction to homological algebra. Cambridge Studies in Advanced Mathematics, 38. Cambridge University Press, Cambridge, 1994.

[57] Wodzicki, M.: Excision in cyclic homology and in rational algebraic K-theory. Ann. Math. 129 (1989), 591639.

Department of Mathematical Sciences, Seoul National University, 1 Gwanak-ro, Gwanak-gu, Seoul 08826, Republic of Korea

Current address: School of Mathematics, Sichuan University, No. 24 South Section 1, Yihuan Road, Chengdu, PR China, 610065

E-mail address: ponge.math@icloud.com 